SLAC- -376

DE91 012188

\title{
FINAL FOCUS TEST BEAM
}

\author{
PROJECT DESIGN REPORT \\ Stanford Linear Accelerator Center \\ Stanford Unjersity \\ Stanford, California $9+309$
}

Marclı 1991

Prepared for the Department of Energy

under contract number DE-AC03-76SF00515

Printed in the Enited States of Ancrica. Avalable from the National Techni(a) Juformation Service, L.S. Department of Commerce, 5285 Port Royal Road, springficiel. Iirginia 22161. 


\section{The Final Focus Test Beam Project}




\section{The Final Focus Test Beam Project}

One of the greatest challenges we face on the road to the Next Linear Collider (NLC) is to make particle beams with extremely small sizes. Whereas the particle bunches in the SLC are millimeter-long needles 4 to 5 microns across, those in the NLC will have to be ten times shorter and up to a thousand times narrower. Such tiny beams are needed to produce luminosities of $10^{33}$ to $10^{34} \mathrm{~cm}^{-2} \mathrm{~s}^{-1}$ that will be necessary to generate sufficient numbers of events as the center-of-mass energy climbs toward $1 \mathrm{TeV}$-and the cross sections for interesting plysical processes drop to the level of about $10^{-37} \mathrm{~cm}^{-2}$.

Other ways to increase the luminosity - e.g., by raising the number of particles per bunch and the machine repetition rate-are limited by the available $\mathrm{AC}$ power and by: interactions of the bunches with each other and with the accelerating structure. Therefore, achieving spot sizes that are a hundred times smaller than the wavelength of visible light will be one of the chief goals in the development of the NLC.

The part of a linear $e^{+} e^{-}$collider that reduces the beam sizes and maintains the beams in collision is called the final focus. Its magnetic elements act much like the lenses of a fine optical telescope to collect the particles produced by the linear accelerator and focus them to a spot with small cross-sectional area. To produce tightly focussed beams and maintain them in collision requires careful control and stabilization of these magnets. and places considerable empliasis on accurate measurement of the properties of the beam itself. We have learned a great deal from operation of the SLC, but successful implementation of future machines at higher energies will demand that even tighter mechanical and electrical tolerances be respected. It will also require greater measurement precision and the development of tuning mechanisms and technicues considerably more powerful than those presently in use.

In collaboration with teanı of physicists and engineers from the Soviet Union, Germany. France, and Japau, we have recently begun to build and instrument a proto1ype magnetic system capable of proclucing the small beam spots required for the NLC. This Final Focus Test Beam (FFT'B) will occupy some 185 meters in the straight-ahead channel at the end of the SLAC: linac with the final clements extending onto the concrete 
surface of the Research Yard. (Figure I.1) As input it will use the unique SLC electron (or positron) beam with its very small emittance. The optics of this beam will be corrected to third order for geometric and chromatic aberrations to produce a focal point at which the beam height will be demagnified by a factor of 300 - to a size smaller than 100 nanometers. Just such a compression factor will be required for the final focus of a $\mathrm{TeV}$-scale linear collider. To attain it we will have to address most of the critical issues inherent in their design, construction and implementation.

The FFTB is truly an international effe. $\{$. The optical design principles were developed by accelerator physicists from around the world, and have been reviewed at major international workshops on linear colliders. Detailed design. error analysis, and development of precise tuning procedures for these optics have been carried out by a group consisting of physicists from KEK (Tsukuba), LAL (Orsay), and SLAC. The magnets were designed and are being fabricated at the Institute for Nuclear Physics (Novosibirsk) in the Soviet Union, and those for the final lens pair are being designed there and in Japan. State-of-the-art mechanical stabilization of these critical components will be done by the group at KEK.

Precise mechanical alignment and stabilization are necessary also for the remainder of the magnetic elements and electronic monitors. They must be aligned to better than 30 microns along the entire test beam, and their position must be stable to 1 to 2 microns in order to maintain the final spot size. Groups from DESY (Hamburg) and SLAC are designing and constructing liardware to achieve these goals.

Improvements, and in some cases completely new directions, in beam diagnostic instrumentation are needed to measure the properties of the beam accurately throughout the system. Measurement of the final spot size is a particularly challenging problem. as the tightly-focused beam will destroy any material in its path. Physicists and engineers at Orsay and SLAC are currently attacling these problems. Beam position monitors and associated electronics are being designed to provide resolutions of $\mathrm{J}$ to 2 nicrons. Wire scanners will be used to measure bcam profiles at locations other than the focal point of the system, and techniques are being developed to measure the focused spot by observing the interaction of the beam wit! gas-jet or renewable-solid targets. 
The Final Focus Test Beam is well underway. Completion of construction is expected in late 1992, and successful commissioning of the test beam will be a major step in the worldwide effort to design and build a TeV-scale linear collider. 


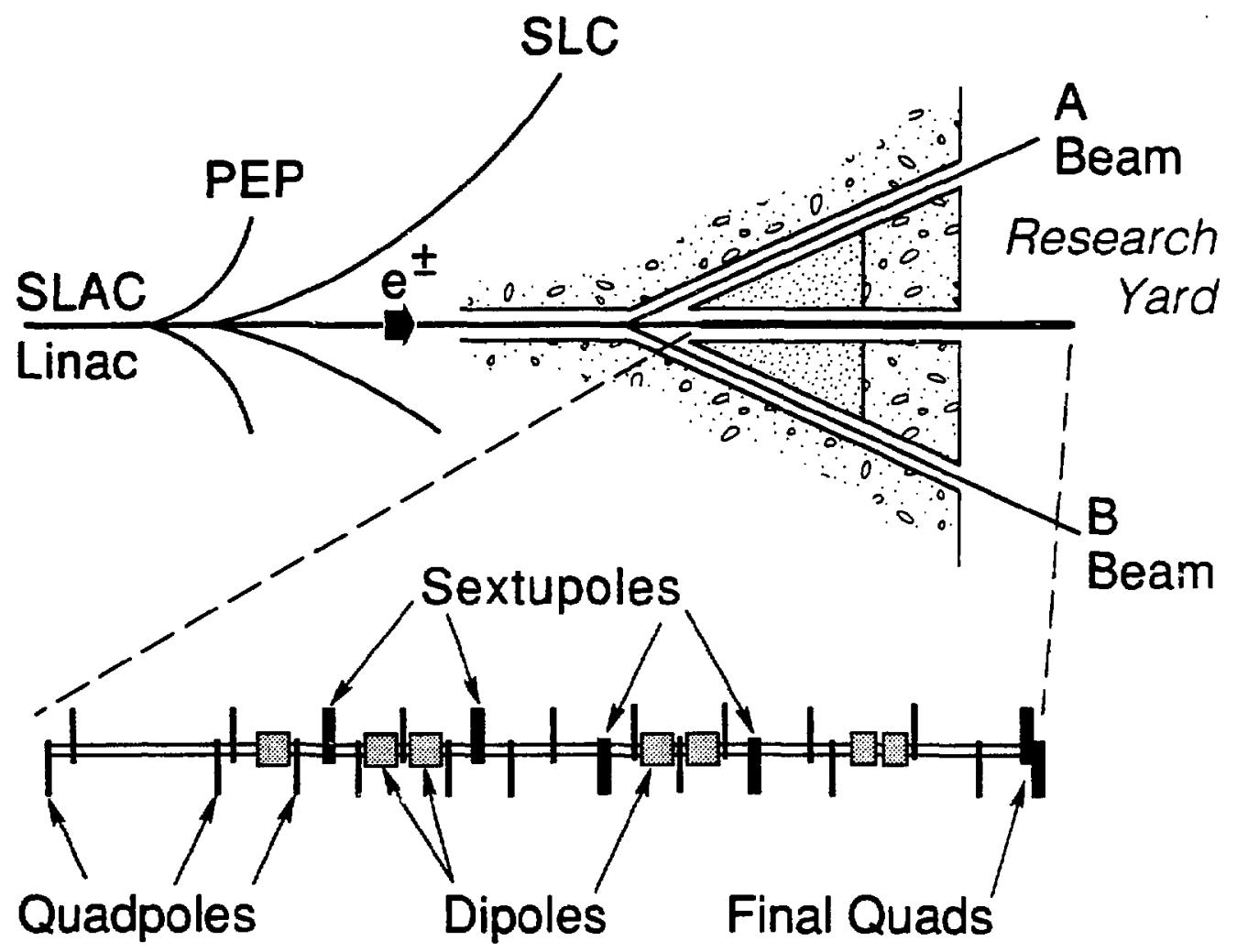

Figure I.1. The Final Focus Test Beam. 


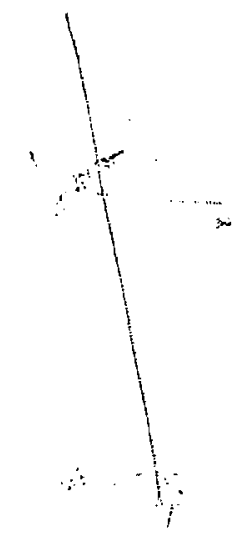

II. Optical Design 


\section{Optical Design}

\section{II.A Introduction}

The Final Focus Test Beam is intended to be a facility to be used for the development and study of the optical systems, instrumentation, and techniques needed to produce the small beam spots required for future $e^{+} e^{-}$linear colliders. A brief description of the parameters and optical design of the FFTB is given in this section of this report. A detailed account of the optical properties and tuning strategies for the beam line can be found in the Final Focus Test Beam Optical Handbook.

The parameters of the FFTB have been chosen to match as closely as possible those of a future linear collider. Table II.1 contains a summary of these paraıneters, and compares them witl those of the SLC Final Focus as well as those used in a typical NLC design. The FFTB will use as input the $50 \mathrm{GeV} \mathrm{SLC} e^{-}$beam from the SLAC linac. The SLC damping ring can produce an invariant emittance of $\gamma \epsilon_{y}=7 \times 10^{-7} \mathrm{rad} \cdot \mathrm{m}$ at quantum mechanical equilibrium when operated with the horizontal and vertical phase space components decoupled from each other (decoupled tunes). The optics of the FFTB, shown schematically in Figure II.A.1, would theoretically be able to reduce the beam to a spot with vertical height $27 \mathrm{~nm}$, but the invariant enittance of the beam will surely be enlarged as the beam is accelerated in the linac. It is expected that, for beam intensities up to $10^{10}$ particles per pulse, this growth can be controlled well enough to deliver $\gamma \epsilon_{y}=3 \times 10^{-6} \mathrm{rad} \cdot \mathrm{m}$ to the entrance of the FFTB. This is sufficiently small that the FFTB will be able to achieve a vertical spot dimension of $60 \mathrm{~nm}$ while maintaining a horizontal size of 1 micron.

The FFTB contains five optical sections. (See Figure II.A.1.) The bean that appears at the end of the linac is first matched to the lattice of the FFTB beam line. The matching section controls the launch of the beam orbit into the FFTB, and contains lenses to match the betatron space of the bean to the FFTB lattice at the entrance to the chromatic correction section. Chromatic correction is done separately in the horizontal and vertical planes. A second $\beta$-matching section lies between the two chronatic correction sections. The overall clemagnification of the system is controlled by the focal lengths of the initial betatron matich and of the final telescopic section. 
A round spot $(1 \mu \times 1 \mu)$ can also be made at the focal point of the system by coupling the damping ring optics and adjusting the strengths of the matching quadrupoles at the entrance of the FFTB. This configuration will be especially useful in the early tuning stages, and later for testing new instrumentation and new focusing elements.

\section{II.B Telescopes}

The basic optical system used to demagnify the transverse dimensions of a beam is a telescope. (See Figure II.B.1.) A simple telescope produces point-to-point and parallelto-parallel imaging together with a geometric demagnification $\frac{1}{N_{x}}=\frac{f_{0}}{f^{*}}=\left(\frac{\beta_{0}}{\beta^{*}}\right)^{1 / 2}$. In this expression, the parameters $\beta_{0}$ and $\beta^{*}$ are the values of the betatron function at the entrance and the focal point of the telescopic system. The one-dimensional matrix representation of such a module is:

$$
R_{x}=\left(\begin{array}{cc}
M_{x} & 0 \\
0 & 1 / M_{x}
\end{array}\right) .
$$

Although the FFTB is not built with strictly telescopic blocks, the first order transformation matrix between the end of the linac and the focal point has this form with $1 / M_{x} \approx 70$ and $1 / M_{y} \approx 380$. The design allows a reduction in the overall length of the system compared to a rigorously telescope-based final focus, but contains only one image point of the focal point for diagnostic and measurement purposes.

\section{II.C Chromaticity}

For a monoenergetic beam with emittance $\epsilon$, the optimized beam size at the focal point is given by,

$$
\sigma^{* 2}=\epsilon \beta^{*}
$$

Unlike this ideal case, however, the beam produced by a linear accelerator contains particles with a finite spread in their energy distribution. Since particles with different energies are focused to different points along the beam line, there are chromatic aberrations in the final spot that must be compensated by additional elements in the magnetic lattice. 
For the simple telescope shown in Fig. II.B.1, the spot size is ${ }^{1}$,

$$
\begin{gathered}
\sigma^{* 2}=\epsilon \cdot \beta^{*}(\delta) \\
\text { with } \beta^{*}(\delta)=\beta^{*}(0)+C_{2} \frac{\delta^{2}}{\beta^{*}(0)}+\text { higher order terms. }
\end{gathered}
$$

As the focusing strength of the system is increased $\left(\beta^{*}(0)\right.$ decreased), the spot size passes through a minimum after which the chromatic aberrations dominate. (See Figure II.C.1.)

The chromaticity of a beam line is defined as

$$
\xi_{x, y}=\int_{s_{0}}^{s_{1}} K(s) \cdot \beta_{x, y}(s) d s,
$$

where $K=\frac{B_{0}}{a \cdot(B \rho)}$ is the strength of the quadrupoles in the lattice. The parameter $\xi$ is a measure of the severity of the chromatic aberrations introduced by the segment of the beam line between $s_{0}$ and $s_{1}$. The most important contribution to the chromaticity is created in the final doublet where the $\beta$-functions are very large $(\approx 20,000 \mathrm{~m})$ and the fields are very strong. (Pole-tip fields are as large as 1.4 Tesla in the final quads of the FFTB.) This results in an increase of the spot size unless the total chromaticity of the line, or the coefficient $C_{2}$ in Eq. II.C.3, can be reduced to nearly zero.

\section{II.D Chromatic Correction}

To correct the chromaticity of the optics at the final focus, sextupole magnets are introduced in a region where there is transverse horizontal dispersion. A sextupole is a magnet whose focusing strengtl is not uniform but varies linearly with the horizontal distance to the axis. Therefore placing a sextupole in a dispersive region will give the particles a kick proportional to their horizontal distance to the axis which in turn is proportional to their energy. The net effect is to create energy-dependent focusing in the lattice that can be controlled. The sextupole strength, along with the dispersion and $\beta$-function at the sextupole, are to be adjusted to bring the overali chromaticity of the beam line to zero. Because it is necessary to cancel very large chromaticities, such a system will be quite sensitive to errors and optical tolerances must be held very tightly. 
A sextupole is also known to introduce strong second crder geometric aberrations which could dominate the spot size.The cancellation of these is done $e^{2}$ by placing the sextupoles in pairs scparated by a $-I$ transformation (a $\pi$ phase advance module with magnification equal to 1). The sccond-order gcometric aberrations then cancel while the chromaticity of the sextupoles can be made to add by manipulating the dispersion function in the $-I$ transform.

Because of the need to control the aspect ratio of the heam at the focal point, and because the horizontal spot size is small enough to be abcrration sensitive, we must correct the chromaticity in the horizontal plane as well. The FFTB design has two cliromatic correction scctions, one for eaclı plane so the clromaticities can be tuned scparately and the sextupoles are always at their optimal location ( $N \pi$ phase advance to the focal point with $N$ integer). The lattice includes a " $\beta$-exclsanger" to match the $\beta$-function from one section to the othcr. It is also a $\pi$ phase advance module. Note that this two-plane correction scheme increases the total length of the system and the number of components required.

This scheme is the basis for chromaticity correction for all magretic focusing systems, has already be . successfully used at the SLC. It requires the introduction of sextupoles and bending magnets which in turn create third and higher orcler aberrations.

\section{I?.E Residual Aberrations and Limits on the Spot Size}

There are two major third-order aberrations that remain in the spot produced by the above chromatic correction procedure. The first is created by the finite thickness of the sextupole magnets which generates third order gcometric aberrations (octupolelike). The fractional increase in the spot size at the focal point is,

$$
\Delta_{s}^{2}=\frac{\Delta \sigma_{y}^{* 2}}{\sigma_{y}^{* 2}}=\frac{5}{12} \cdot k_{s}^{4} \cdot l_{s}^{2} \cdot \epsilon_{y}^{2} \cdot \beta_{y}^{4}
$$

where $k_{s}$ is the integrated strength of the sextupole. For the FFTB, $k_{s} \approx 20 \mathrm{~m}^{-2}, l_{s}=$ $.25 \mathrm{~m}$, and $\beta_{y}=12,000 \mathrm{~m}$ at the sextupole in the vertical chromatic correction section:

$$
\Delta_{s}^{2}=0.08
$$

This corresponds to an increase of the spot size of the orcler of $4 \%$. 
The second effect that limits the spot size is more subtle, but just as significant. As particles pass through the bend magnets that create the dispersion necessary for the chromatic correction to occur, they lose some energy through synchrotron radiation. The cancellation between the chromaticity introduced by the sextupoles and that introduced by the final quad can no longer be rigorous. There is an increase of the spot size given by,

$$
\Delta_{b}^{2}=\frac{\Delta \sigma_{y}^{* 2}}{\sigma_{y}^{* 2}}=\frac{55}{10 \sqrt{3}} \cdot \gamma_{c} \cdot \lambda_{c} \cdot \gamma^{5} \cdot \frac{\theta_{b}^{3}}{l_{b}^{2}} \cdot \xi_{y}^{2} .
$$

From the sextupole setting, $\zeta_{y}=2 k_{s} \eta_{s} \beta_{s} \approx 2 \times 10^{4}$. In the case of the FFTB,

$$
\Delta_{b}^{2}=0.13
$$

This corresponds to an increase of the spot size of $7 \%$.

The optimal design ${ }^{3}$ that minimizes the spot dilution from these two effects occurs witl the parameters $l_{b}=5.5 \mathrm{~m}, \theta_{b}=7.8 \mathrm{mrad}$, but constraints imposed by saturation of the steel material in the sextupoles, and the desire to place the entire chromatic correction system inside the BSY (without extending onto the Research Yard), result in a compromise solution with $l_{s}=25 \mathrm{~cm}$ and $l_{b}=5.25 \mathrm{~m}$. The bend angle for the beam line becomes $\theta_{b}=7.4 \mathrm{mrad}$, and the spot dilution is

$$
\Delta_{0}^{2} \equiv \Delta_{s}^{2}+\Delta_{b}^{2}=0.21
$$

A similar optimization, that includes the emittance growtl due to synchrotron radiation in the bend magnets, is done for the horizontal plane. Synchrotron radiation in the bends will increase the horizontal emittance of the beam, but will not affect the rertical emittance as long as there is no coupling introduced by the lattice.

Synchrotron radiation in the final quadrupole lenses will ultimately place an inescapable limit on the final spot size. This effect, known as the Oide limit, sets an upper 
bound on the focusing of an electron beam that depends primarily on the normalized enuttance $\gamma \epsilon_{y}$ :

$$
\sigma_{y}^{*}=\left(\frac{7}{5}\right)^{\frac{1}{2}}\left[\frac{275}{3 \sqrt{6 \pi}} r_{e} \lambda_{i} F\left(\sqrt{K} l_{Q}, \sqrt{h} l^{*}\right)\right]^{\frac{1}{7}}\left(\gamma \epsilon_{y}\right)^{\frac{\rho}{7}} .
$$

The function $F$ is a dimensionless function of the length and strength of the final quadrupole and the focal length of the systen. This is not a limitation for the FFTB. but becomes significant for future linear collider final focus systems. 


\section{References}

1. Karl Brown, SLAC PUB-4159, (1987).

2. Kar] Brown and Roger Servianckx, SLAC-PUB-3381, (1984).

3. K. Oide, SLAC-PUB-4953, (1989). 
Table II.1. Parameters of the Final Focus Test Beam

\begin{tabular}{|c|c|c|c|}
\hline Parameter & NLC & SLC & FFTB \\
\hline Beam energy: $(\mathrm{TeV})$ & 0.25 to 0.75 & 0.0 .5 & 0.05 \\
\hline Enittance: $\quad \gamma \epsilon_{y}(\mathrm{rad}-\mathrm{m})$ & $3 \times 10^{-8}$ & $3 \times 10^{-5}$ & $3 \times 10^{-6}$ \\
\hline Focusing: $\quad \beta_{y}^{*}(\mu \mathrm{m})$ & 100 & 7000 & 100 \\
\hline Demagnification: & 300 & 30 & 380 \\
\hline Beam height: $\sigma_{y}(\mathrm{~nm})$ & 3 to 5 & 2000 & 60 \\
\hline Aspect ratio: & 100 to 200 & 1 & 15 \\
\hline Bunch length: $\sigma_{z}(\mu \mathrm{m})$ & 50 to 100 & 500 & 500 \\
\hline Bandwidth: $\delta p / p(\%)$ & \pm 0.3 & \pm 0.3 & \pm 0.3 \\
\hline Bunch population $\left(10^{10}\right)$ & 1 to 2 & 3 to 5 & 1 to 2 \\
\hline . & & & \\
\hline
\end{tabular}



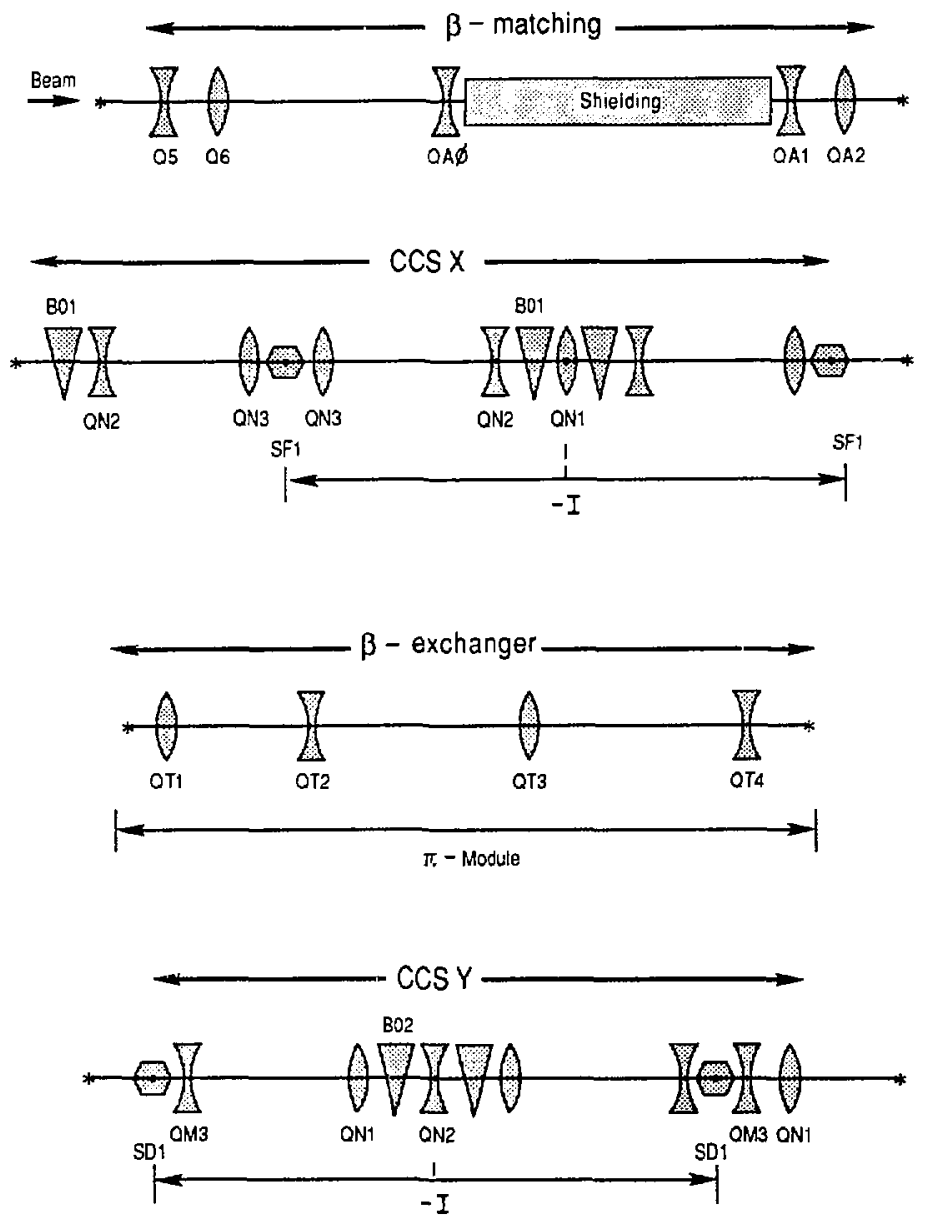

Final Transformer

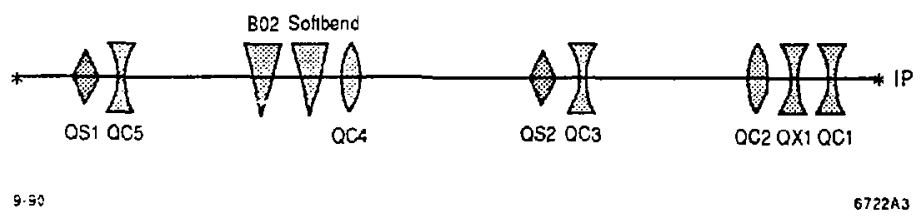

Fignure II.A.I. Schematic representation of the optics of the FFTB magnetic lattice. 


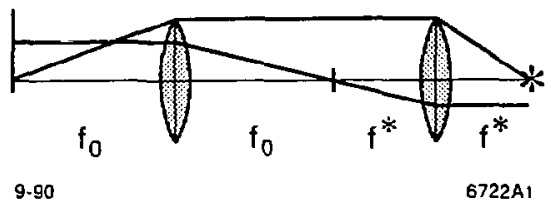

Figure II.B.1. A simple two-lens optical telescope.

II-11 


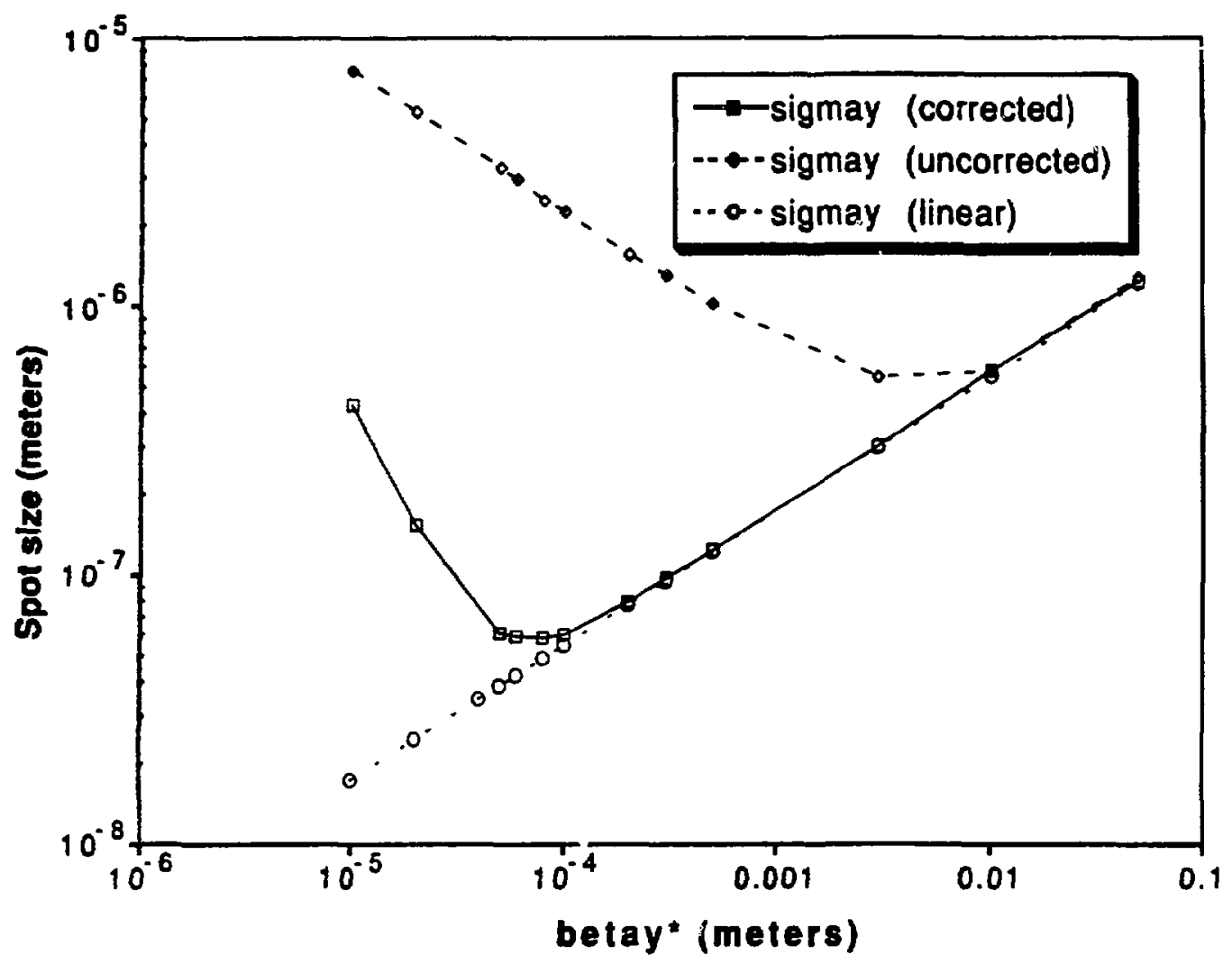

Figure II.C.1. Variation of the vertical beam height at the focal point of the FFTB as the demagnification $\left(\beta_{y}^{*}\right)$ of the optics is changed. The dotted curve is the lowest order geonetric beam size, while the dashed curve includes uncorrected chromatic aberrations. The solid curve is the size expected for the FFTB after second-order chromatic corrections are applied. 
III. Magnets 


\section{Magnets}

\section{III.A Magnet Specifications}

The FFTB beam line consists of discrete dipole, quadrupole, and sextupole magnetic elements. A detailed list of these elements and typical operating points for them (at $50 \mathrm{GeV}$ beam energy) are given in Table III.1.

A summary of the number of each magnet type required for the system is given in Table 111.2. Spccifications for the apertures and harmonic content of each magnet 1ype are also included in Table III.2. The apertures have been defined to assure at least $10 \sigma$ clearance between the pole-tips of the elcments and the nominally $\beta$-matched beam with design emittance. Tolerances on the hamonic content of the fields have been calculated by placing a linit of $2 \%$ on the dilution (per magnet) of the final spot size due to imperfections in the fields.

\section{III.B Magnet Design and Fabrication}

Designs for all magnet types have been created by the group at the Institute for Nuclear Physics in Novosibirsk in cooperation with engineers and physicists at SLAC. The designs are based on paramelers common to existing SLC magnets, but some modifications have been made to provide a better fit to the needs of the FFTB. Some of the features are taken from the design of the VEPP4 magnets. All of these magnets will be fabricated in the Soviet Union, tested there, and then shipped to SLAC. Further testing and calibration will be done at SLAC before the magnets are installed on the beam line.

The group at KEK will design and fabricate a version of the quadrupoles that make up the final leus doublet (Q(2/QX1/QC!1), and will provide a mechanically stabilized support structure for them.

\section{Quadrupoles}

The optical design of the FFTB has been tailored so that 28 of the 39 quadrupoles reguired for the system can be constructed from a single mechanical design-the socalled "Standard Quadrupole". An assembly drawing of this magnet is shown in Figure III.B.I. It is a solicl core magnet with an effective magnetic length of $46.1 \mathrm{~cm}$ and bore 
diameter of $2.3 \mathrm{~cm}$ that will be operated with pole-tip fields below $10 \mathrm{kC}$ in all cases. The harmonic content of this magnet has been analysed with the progran POISSON. With reasonable construction tolerances, this design will easily achieve the $0.1 \%$ restriction on all nonquadrupole fields given in Table III.2.

The coils for the Standard Quadrupole will be wound from proinsulated $4.8 \mathrm{~mm}$ square copper conductor cooled by water flow in a passage through its center. The design is shown in Figure III.B.2. The coils will be vacuum impregnated with epoxy to provide a watertight seal. Trim coils wili be wound on the four back legs of the quadrupole profile. These can be powered independently to simultaneously provide up to $\pm 10 \mu \mathrm{m}$ motion of the magnetic center of the field and $\pm 4 \%$ adjustment of the quadrupole strength.

A second quadrupole design is used for one of the large-aperture magnets in the final telescope of the system (QC3) and for several of the lenses in the dump line downst rean o. the focal point. This design is a scaled rersion of the Standard Quadrupole.

The lenses of the final doublet (QX1/QC1) are more difficult to design and fabricate than the remainder of the quadrupole elements. The restrictions on their harmonic content are somewhat tight, and in some cases, these magnets must operate with poletip fields that are as large as $14 \mathrm{kG}$. The alignment tolerances for these elements are such that they are best designed and fabricated as a complete set, and that they be supported by a common mounting structure. The group at KEK will design and falsricate a version of these magnets along with their supports. A prototype magnet wilh pole-tips made from the composite material Permendur is presently being labricated, and is expected to lead to a successful design of a full scale set of lenses.

\section{Dipoles}

The aipole bending magnets for the FFI'B are all of a single design---the so-called "Standard Dipole". This is a solid-core "H"-type magnet with relatively modest field strengths and tolerances. An assembly drawing of the Norosibirsk design is shown in Figure III.B.3. Each magnet is 2.8 meiets long with $2.3 \mathrm{~cm}$ gap spacing. In several locations along the beam line. lor example at the soft bends near the focal point, it is necessary to insert an iron spacer in the back legs of these magnets to increase $1 l_{10}$ 
pole-tip aperture. This is easily done with negligible loss in field quality in the region of interest.

Tlıe coils are wound from water-cooled preinsulated $8.6 \mathrm{~mm}$ square copper conductor, and will be vacuum impregnated witl epoxy. Trim windings provide $\pm 3 \%$ range in the strengtl of the individual coils.

These magnets are used in pairs spaced slightly apart to create the bends in the beam line. This provides access to the center of each bend, or hinge, point in the lat tice.

\section{Sextupoles}

All sextupole magnets in the FFTB are of a single design, and will be fabricated in the Soviet Union. The assembly drawing is shown in Figure III.B.4. The harmonic content of these magnets is easily within the design criteria, but to avoid severe saturation of the magnetic field in the pole-tip, it has been necessary to reduce the aperture of these magnets to $2.06 .5 \mathrm{~cm}$ and set their overall length to $25 \mathrm{~cm}$. The magnet steel will remain slightly saturated $(\approx 5 \%)$ at $50 \mathrm{GeV}$ beam energy, and there is some dilution of the performance of the system due to the length of the magnets. The effect on the spot size of the thick-lens sextupole lias been cliscussed in Section II of this report.

\section{III.C Testing and Operation}

Beforc the magnets are shipped to SLAC from the Soviet Union, the magnetic field of each unit will be measured to assure lloat the desired tolerances have been met. The inagnets will be recalibrated at SLAC. In the case of the quadrupole magnets, this will occur after the Beam Position Monitors have been inserted through the pole tips. Section $V$ of this report provide further details of the magnetic measurement procedures to be used at this step.

A series of tests will be performed on the coils prior to their assembly into the magnets in the Soviet Union. This will include a check of the resistance and a high-pot test of each coil. The integrity of the conling circuit will be checked with water at high pressure, and measurements will be made of the water flow rate through the coil at the nornal operating pressure differential. 
'To protect the coils against possible failure during normal scrvice, thermal switches are mounted directly onto the copper conductor at the outlet of the cooling circuit, and flow switches will be used to verify the supply of cooling water. 


\begin{tabular}{|c|c|c|c|c|c|c|c|c|c|c|c|c|c|c|c|c|c|}
\hline & & & & & & & * & $*$ & + & + & + & & & & & & \\
\hline 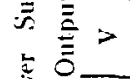 & & F & & \% & $\approx$ & $\stackrel{10}{\dddot{N}}$ & 哭 & 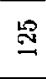 & $\stackrel{\Xi}{\Xi}$ & $\stackrel{\llcorner}{\stackrel{0}{二}}$ & $\stackrel{\mathfrak{g}}{\Im}$ & 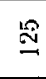 & F & F & P & $F$ & 유 \\
\hline$\stackrel{\overline{0}}{-}<$ & 蛋 & 怘 & 온 & 空 & 总 & 우 궁 & 음 & \% & 융 & 융 & 유 & 8 & 益 & 品 & 点 & 喿 & 吕 \\
\hline 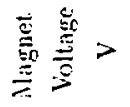 & $\stackrel{\sim}{2}$ & $\infty$ & 0 & 可 & $I$ & $\stackrel{19}{S}$ & $\Xi$ & $\approx$ & $\stackrel{\mathrm{N}}{\mathrm{N}}$ & 獣 & $\stackrel{S}{S}$ & 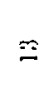 & $\vec{\nabla}$ & 1 & \pm & $\stackrel{D}{N}$ & 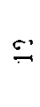 \\
\hline 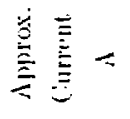 & $\supseteq$ & $\ominus$ & $\approx$ & Э્ગ & $\Xi$ & ํㅗㅇ & $\stackrel{\Xi}{\varrho}$ & 5 & $\stackrel{18}{8}$ & 总 & 谷 & 巨 & $\stackrel{g}{ }$ & $\Sigma$ & $\Xi$ & $\stackrel{\nabla}{\vec{N}}$ & § \\
\hline$\stackrel{亏}{\Xi} \cong$ & $\stackrel{5}{5}$ & 崩 & $\stackrel{5}{\circ}$ & $\stackrel{15}{2}$ & $\stackrel{\hat{\mathscr{E}}}{\sigma}$ & $\underset{\infty}{\infty}$ & 8 & $\stackrel{\tilde{\sigma}}{\nabla}$ & $\begin{array}{l}\hat{\infty} \\
\infty\end{array}$ & $\underset{\infty}{\infty}$ & $\begin{array}{l}2 \\
\infty \\
\infty\end{array}$ & $\underset{2}{0}$ & $\underset{1}{\stackrel{2}{2}}$ & $=$ & $\exists$ & $\stackrel{8}{0}$ & 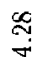 \\
\hline 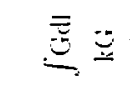 & & 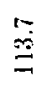 & $=$ & $\stackrel{\vec{\partial}}{0}$ & 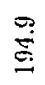 & 莣 & 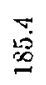 & $\begin{array}{l}\square \\
\vdots \\
\stackrel{2}{二}\end{array}$ & $\stackrel{0}{\stackrel{5}{0}}$ & $\frac{9}{15}$ & $\underset{0}{0}$ & $\underset{\dot{S}}{\mathscr{S}}$ & $\begin{array}{l}\checkmark \\
\underset{D}{0} \\
:\end{array}$ & 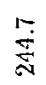 & 䣸 & 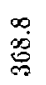 & $\stackrel{9}{E}$ \\
\hline 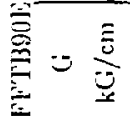 & & $\stackrel{5}{\stackrel{i}{i}}$ & \begin{tabular}{l}
$\vec{D}$ \\
\multirow{2}{*}{}
\end{tabular} & $\stackrel{\sim}{\infty}$ & 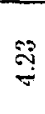 & $\overbrace{1=0}^{\infty}$ & $\stackrel{\sim}{\circ}$ & $\underset{\stackrel{N}{O}}{\sigma}$ & $\underset{0}{\infty}$ & $\stackrel{8}{6}$ & $\stackrel{\infty}{:}$ & $\stackrel{N}{*}$ & $\stackrel{0}{0}$ & $\overrightarrow{0}$ & ह5 & $\underset{\infty}{8}$ & $\stackrel{N}{i}$ \\
\hline$\Xi \Xi$ & $\vec{\theta}$ & $\vec{\Phi}$ & $\vec{\Phi}$ & $\vec{\oplus}$ & $\vec{\Leftrightarrow}$ & $\vec{E}$ & $\ddot{\vartheta}$ & $\overrightarrow{0}$ & $\ddot{\circ}$ & $\dot{0}$ & $\overrightarrow{0}$ & $\overrightarrow{0}$ & $\overrightarrow{5}$ & $\overrightarrow{5}$ & $\ddot{6}$ & $\overrightarrow{0}$ & $\overrightarrow{\dot{\theta}}$ \\
\hline 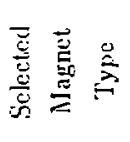 & 㣻 & $\begin{array}{l}\frac{1}{2} \\
\frac{0}{0} \\
\stackrel{5}{0}\end{array}$ & $\frac{\Xi}{5}$ & 芒 & 芯 & 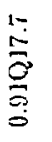 & 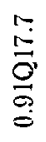 & 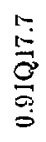 & 总 & $\frac{\dddot{Z}}{\stackrel{\Xi}{8}}$ & 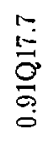 & 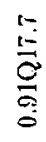 & 莺 & 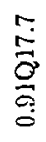 & 点 & $\begin{array}{l}\text { 点 } \\
9 \\
9 \\
9\end{array}$ & 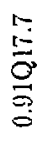 \\
\hline $\bar{\Xi} \quad \bar{\Xi}$ & $\stackrel{\check{g}}{5}$ & $\stackrel{\Xi}{\leftrightarrows}$ & $\stackrel{8}{\circ}$ & $\stackrel{\varrho}{0}$ & $\stackrel{8}{8}$ & $\stackrel{\bar{\Xi}}{=}$ & $\stackrel{\circ}{8}$ & $\begin{array}{l}8 \\
8 \\
8\end{array}$ & $\stackrel{8}{8}$ & $\stackrel{8}{:}$ & $\stackrel{8}{8}$ & $\stackrel{8}{\check{\Xi}}$ & 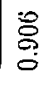 & $\begin{array}{l}\mathscr{8} \\
\stackrel{8}{\circ}\end{array}$ & $\stackrel{8}{8}$ & $\stackrel{8}{8}$ & $\stackrel{\wp}{\mathscr{\delta}}$ \\
\hline 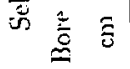 & $\underset{\sim}{\stackrel{\sim}{~}}$ & $\underset{p}{\stackrel{p}{0}}$ & 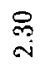 & 吊 & $\stackrel{\overbrace{}}{\text { ণ }}$ & 尽 & 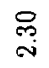 & 品 & $\stackrel{\overbrace{}}{\stackrel{\rho}{\sim}}$ & 吊 & 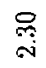 & 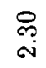 & $\stackrel{8}{\mathscr{r}}$ & 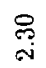 & ஜ্ণ & 品 & $\stackrel{\circ}{\sim}$ \\
\hline$\stackrel{\Xi}{\Xi} \cong$ & $\underset{\sigma}{0}$ & $\stackrel{\vec{Z}}{\vec{i}}$ & $\stackrel{\overbrace{}}{0}$ & $\underset{\infty}{\infty}$ & $\stackrel{\varrho}{\circ}$ & $\underset{\not \infty}{0}$ & $\stackrel{8}{\overparen{7}}$ & 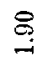 & $\stackrel{\Xi}{0}$ & $\stackrel{\leftrightarrow}{0}$ & J্ّ & 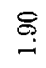 & $\stackrel{\infty}{\infty}$ & $\stackrel{\hat{H}}{0}$ & $\stackrel{0}{\circ}$ & $\stackrel{9}{\longrightarrow}$ & กิ \\
\hline$\stackrel{8}{0}$ & $\stackrel{0}{0}$ & $\stackrel{0}{\circ}$ & $\overrightarrow{0}$ & $\stackrel{5}{\circ}$ & $\stackrel{\Xi}{0}$ & ণุ & $\frac{\cong}{0}$ & $\stackrel{\dddot{y}}{0}$ & $\stackrel{8}{0}$ & $\stackrel{1}{8}$ & ญ్ & $\frac{2}{0}$ & $\frac{m}{0}$ & $\begin{array}{l}\mathscr{8} \\
0 \\
0\end{array}$ & $\stackrel{\mathscr{S}}{0}$ & $\stackrel{8}{0}$ & $\stackrel{8}{8}$ \\
\hline 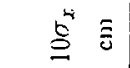 & $\bar{a}$ & $\stackrel{\substack{19 \\
g}}{9}$ & $\stackrel{0}{0}$ & $\stackrel{\square}{\square}$ & $\stackrel{\dddot{乛}}{0}$ & $\bar{\square}$ & $\stackrel{2}{\mathscr{g}}$ & 29: & $\tilde{\overbrace{}}$ & $\stackrel{\infty}{0}$ & $\stackrel{\mathscr{D}}{0}$ & $\stackrel{2}{9}$ & $\overrightarrow{5}$ & $\stackrel{\overbrace ึ}{0}$ & $\stackrel{\sim}{\sigma}$ & 怘 & $\stackrel{8}{0}$ \\
\hline$\cong$ & 8 & $\widetilde{\sigma}$ & $\stackrel{\circ}{8}$ & $\sigma$ & $\stackrel{0}{\square}$ & z & 菅 & 彎 & $\stackrel{\searrow}{\circlearrowright}$ & $\underset{c}{z}$ & $\stackrel{8}{\circ}$ & 澹 & $=$ & $\mathscr{\sigma}$ & $\stackrel{0}{0}$ & 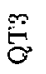 & 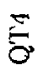 \\
\hline
\end{tabular}




\begin{tabular}{|c|c|c|c|c|c|}
\hline & $\triangleright \triangleleft \triangleleft \triangleleft \triangleright \triangleright \triangleleft$ & & & & $000 \square+$ \\
\hline $\begin{array}{l}E_{i} \\
\end{array}$ & 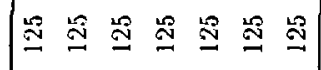 & 웅 우 & & & क \\
\hline$=$ & 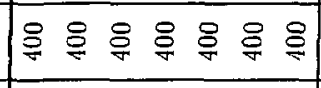 & 总点品 & & & 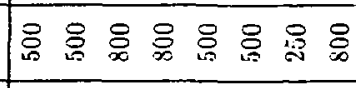 \\
\hline 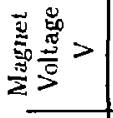 & 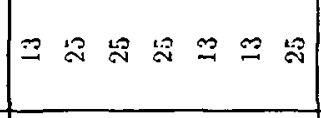 & 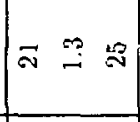 & & & 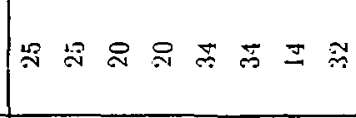 \\
\hline & 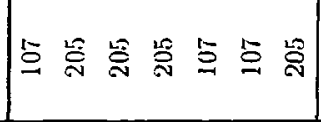 & 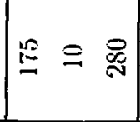 & & & 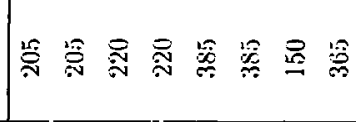 \\
\hline 0 & 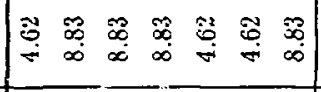 & 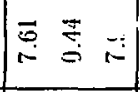 & $\stackrel{=}{=}$ & 皇 & 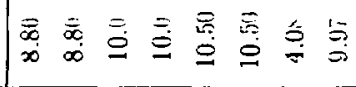 \\
\hline$\underbrace{\overline{5}}$ & 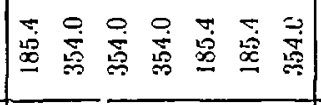 & 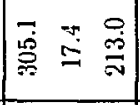 & 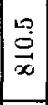 & 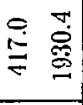 & 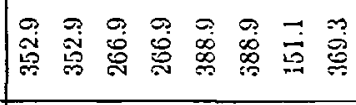 \\
\hline$=\frac{\bar{a}}{3}$ & 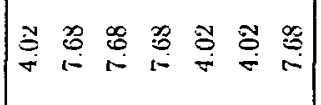 & 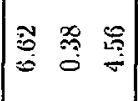 & 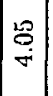 & 里瓷 & 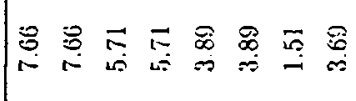 \\
\hline 氞它 & 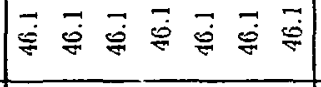 & 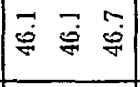 & 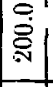 & 邑兰 & 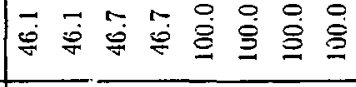 \\
\hline 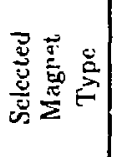 & 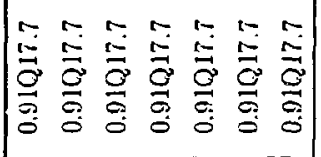 & 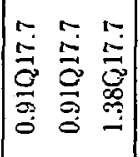 & $\mid$ & 存离 & 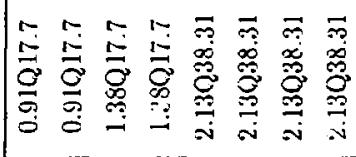 \\
\hline 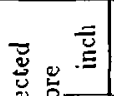 & 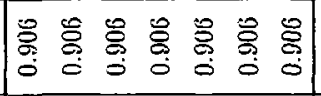 & 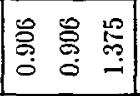 & 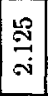 & $\begin{array}{ll}0 \\
0\end{array}$ & 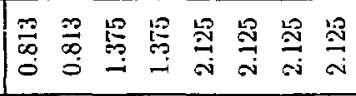 \\
\hline 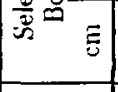 & 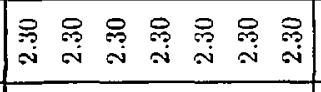 & 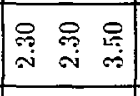 & \begin{tabular}{|l|} 
\\
9 \\
50 \\
50
\end{tabular} & 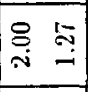 & 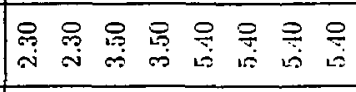 \\
\hline 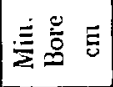 & 원 용 & 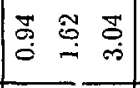 & : & 品 & 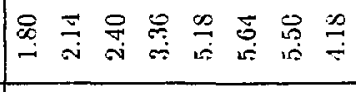 \\
\hline$\stackrel{8}{9} \overline{5}$ & 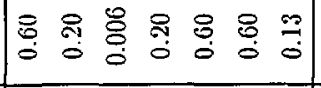 & 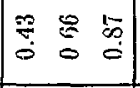 & \begin{tabular}{|l|l|} 
\\
0 \\
\end{tabular} & 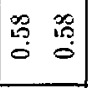 & 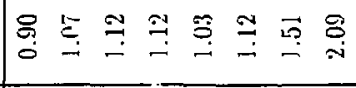 \\
\hline$\stackrel{0^{4}}{\Xi}$ & 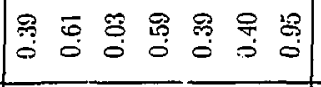 & 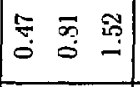 & \begin{tabular}{|c|}
5 \\
$i$ \\
\end{tabular} & $\stackrel{\square}{\leftrightarrows}$ & 施 \\
\hline & 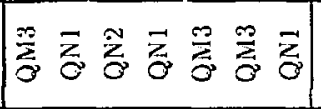 & {$\left[\begin{array}{lll}5 & 0\end{array}\right.$} & & $\overrightarrow{0}$ & 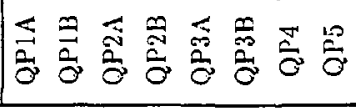 \\
\hline
\end{tabular}


Table III.IC FF'B Dipole Magnets

\begin{tabular}{|c|c|c|c|c|c|c|c|c|c|c|c|c|c|c|}
\hline \multirow{3}{*}{$\begin{array}{l}\text { Nagnet } \\
\text { II) }\end{array}$} & \multirow{3}{*}{$\begin{array}{l}10 \sigma_{x} \\
\operatorname{con}\end{array}$} & \multirow{3}{*}{$\begin{array}{c}10 \sigma_{y} \\
\mathrm{cln}\end{array}$} & \multirow{3}{*}{$\begin{array}{l}\text { Ninl. } \\
\text { (ialp } \\
\text { cull }\end{array}$} & \multirow{2}{*}{\multicolumn{2}{|c|}{$\begin{array}{c}\text { Selected } \\
\text { Gialp }\end{array}$}} & \multirow{3}{*}{$\begin{array}{l}\text { Selected } \\
\text { Magnet. } \\
\text { lype }\end{array}$} & \multirow{3}{*}{$\begin{array}{c}\text { Piffective } \\
\text { Length } \\
m\end{array}$} & \multirow{3}{*}{$\begin{array}{l}\int_{k i m}^{l i l l} \\
\text { lim }\end{array}$} & \multirow{3}{*}{$\begin{array}{l}\text { J'bool: } \\
\text { (ia) Flux } \\
\text { kei }\end{array}$} & \multirow{3}{*}{$\begin{array}{c}\text { Approx. } \\
\text { Current } \\
\lambda\end{array}$} & \multirow{3}{*}{$\begin{array}{c}\text { Magnot } \\
\text { Voltager } \\
V\end{array}$} & \multirow{2}{*}{\multicolumn{3}{|c|}{$\begin{array}{c}\text { Power Sinply } \\
\text { Output }\end{array}$}} \\
\hline & & & & & & & & & & & & & & \\
\hline & & & & cin & inch & & & & & & & $\Lambda$ & V & \\
\hline $\mathrm{BO} 1 \mathrm{~A}$ & 0.29 & 0.09 & 0.17 & 2.30 & 0.9 & 3.940102 .4 & 2.62 & 6.171 & 2.353 & 365 & (9.0) & 400 & 125 & $*$ \\
\hline BOII3 & 0.355 & 0.17 & 0.31 & 2.30 & 0.9 & 3.940102 .1 & $2.6 \%$ & 6.171 & 2.35 .3 & 3365 & 9.0 & 100 & 125 & * \\
\hline BOIC & 0.28 & 0.18 & 0.36 & 2.30 & 0.9 & $3.91 \mathrm{D} 102.4$ & 2.62 & 6.171 & 2.353 & 365 & 9.0 & 100 & 125 & * \\
\hline $\mathrm{BO} 1 \mathrm{D}$ & 0.10 & 0.10 & 0.20 & 2.30 & 0.9 & $3.94 D 102.4$ & 2.62 & 6.171 & 2.35 .3 & 365 & 9.0 & 400 & 125 & * \\
\hline BO1E & 0.19 & 0.10 & .3 & 2.30 & 0.9 & 3.940102 .4 & 2.62 & 6.171 & 2.35 .3 & 305 & 9.0 & 100 & 125 & $*$ \\
\hline BO1F & 0.28 & 0.18 & 0.36 & 2.30 & 0.9 & 3.940102 .4 & 2.62 & 6.171 & 2.35 .33 & 365 & 9.0 & 400 & 325 & $*$ \\
\hline$B O 2 \lambda$ & 0.57 & 0.17 & 0.34 & 2.30 & 0.9 & $3.94 \mathrm{D} 102.4$ & 2.62 & 6.171 & 2.353 & 365 & 9.0 & 400 & 125 & + \\
\hline 130213 & 0.31 & 0.09 & 0.18 & 2.30 & 0.9 & $3.94 D 102.4$ & 2.62 & 6.171 & 2.353 & 365 & 9.0 & 400 & 125 & + \\
\hline $\mathrm{BO} 2 \mathrm{C}$ & 0.29 & 0.09 & 0.18 & 2.30 & 0.9 & $3.94 \mathrm{D} 102.4$ & 2.62 & 6.171 & 2.353 & 365 & 9.0 & 100 & 125 & + \\
\hline $\mathrm{BO} 2 \mathrm{D}$ & 0.55 & 0.17 & 0.34 & 2.30 & 0.9 & $3.94 \mathrm{D} 102.4$ & 2.62 & 6.171 & 2.35 .3 & 365 & 9.0 & 400 & 125 & + \\
\hline $\mathrm{BO} 2 \mathrm{E}$ & 0.20 & 0.54 & 1.08 & 2.30 & 0.9 & $3.94 \mathrm{D} 102.4$ & 2.62 & 0.171 & 2.353 & 365 & 9.0 & 400 & 125 & + \\
\hline $\mathrm{BO} 2 \mathrm{~F}$ & 0.38 & 0.57 & 1.14 & 2.30 & 0.9 & $3.94 \mathrm{D} 102.4$ & 2.62 & 6.171 & 2.353 & 365 & 9.0 & 400 & 125 & + \\
\hline BO $3 A$ & 0.57 & 0.61 & 1.22 & 3.50 & 1.38 & $3.94 \mathrm{D} 102.4$ & 2.64 & 0.806 & 0.305 & 215 & 1.3 & 250 & 10 & $\triangle$ \\
\hline HO3B & 0.76 & 0.61 & 1.28 & 3.50 & 1.38 & $3.94 \mathrm{D} 102.4$ & 2.64 & 0.806 & 0.30 .5 & 215 & 1.3 & " & & $\triangle$ \\
\hline $\mathrm{BO} 4 \mathrm{~A}$ & 1.72 & 2.07 & 3.44 & 3.50 & 1.38 & $3.94 \mathrm{D} 102.4$ & 2.61 & 0.835 & 0.316 & 222 & 1.4 & 250 & 40 & $\nabla$ \\
\hline BO4B & 1.60 & 1.92 & 3.20 & 3.50 & 1.38 & $3.94 \mathrm{D} 102.4$ & 2.64 & 0.835 & 0.316 & 222 & 1.4 & $"$ & $"$ & $\nabla$ \\
\hline BO5A & 1.46 & 1.76 & 2.92 & 3.50 & 1.38 & $3.94 \mathrm{D} 102.4$ & 2.61 & 8.349 & 3.18 .3 & 710 & 18 & 800 & 50 & 0 \\
\hline BOSI3 & 1.30 & 1.61 & 2.60 & 3.50 & 1.38 & $3.94 \mathrm{D} 102.4$ & 2.64 & 8.349 & 3.183 & 740 & 18 & $"$ & $"$ & o \\
\hline
\end{tabular}


Table III.ıD FFTB Sextupole Magnets

\begin{tabular}{|c|c|c|c|c|c|c|c|c|c|c|c|c|c|c|}
\hline \multirow{2}{*}{$\begin{array}{c}\text { Magnet } \\
\text { ID }\end{array}$} & \multirow{2}{*}{$\begin{array}{c}10 \sigma_{x} \\
\mathrm{~cm}\end{array}$} & \multirow{2}{*}{$\begin{array}{c}10 \sigma_{y} \\
\mathrm{~cm}\end{array}$} & \multirow{2}{*}{$\begin{array}{c}\text { Min. } \\
\text { Bore } \\
\mathrm{cm}\end{array}$} & \multicolumn{2}{|c|}{$\begin{array}{c}\text { Selec ied } \\
\text { Bore }\end{array}$} & \multirow{2}{*}{$\begin{array}{c}\text { Selected } \\
\text { Mngnet } \\
\text { Type }\end{array}$} & \multirow{2}{*}{$\begin{array}{l}\text { Effective } \\
\text { Length } \\
\mathrm{cm}\end{array}$} & \multirow{2}{*}{$\begin{array}{l}\text { Strength } \\
\mathrm{kG} / \mathrm{cm}\end{array}$} & \multirow{2}{*}{$\begin{array}{c}\text { PF'ГBУOE } \\
\text { Pole Tip B } \\
k G\end{array}$} & \multirow{2}{*}{$\begin{array}{c}\mathrm{G}^{\prime} \\
\mathrm{kG} / \mathrm{cm}^{2}\end{array}$} & \multirow{2}{*}{$\begin{array}{c}\text { Approx. } \\
\text { Current } \\
A\end{array}$} & \multirow{2}{*}{$\begin{array}{c}\text { Magnet } \\
\text { Voltage } \\
v\end{array}$} & \multicolumn{2}{|c|}{$\begin{array}{c}\text { Power Supply } \\
\text { Output }\end{array}$} \\
\hline & & & & $\mathrm{cm}$ & inch & & & & & & & & $A$ & $v$ \\
\hline $\mathrm{SF} 1 \lambda$ & 0.95 & 0.12 & 1.90 & 2.06 & 0.813 & 0.8159 .44 & 25.00 & 307.6 & 6.558 & 12.30 & 160 & 10 & 250 & 40 \\
\hline SF1B & 0.95 & 0.12 & 1.90 & 2.06 & 0.813 & $0.81 S 9.44$ & 25.00 & 307.6 & 6.558 & 12.30 & 160 & 10 & $"$ & $"$ \\
\hline $\operatorname{SD} 1 \Lambda$ & 0.38 & 0.60 & 1.20 & 2.06 & 0.813 & $0.81 S 9.44$ & 25.00 & 325.1 & 6.931 & 13.00 & 170 & 10 & 250 & 40 \\
\hline SDIB & 0.39 & 0.60 & 1.20 & 2.06 & 0.813 & 0.8159 .44 & 25.00 & 325.1 & 6.931 & 13.00 & 170 & 10 & & $"$ \\
\hline
\end{tabular}


Table III.2. Large Magnet Types for the Final Focus Test Beam

\begin{tabular}{|c|c|c|c|c|}
\hline Magnet Type & $\begin{array}{c}\text { Aperture } \\
(\mathrm{m} m)\end{array}$ & $\begin{array}{l}\text { Length } \\
(\mathrm{cm})\end{array}$ & Number & $\begin{array}{c}\text { Harmonic Tolerance }{ }^{(\alpha)} \\
\qquad(\%)\end{array}$ \\
\hline Standard Quad & 23 & 46.1 & $29^{(b)}$ & 0.1 \\
\hline $1.38 \mathrm{Q} 17.7(\mathrm{QC} 3)$ & 35 & 46.1 & 3 & 0.05 \\
\hline $2.05 \mathrm{Q} 77.7(\mathrm{QC} 2)$ & 5.1 & 200.0 & 1 & 0.03 \\
\hline $0 . S 0 Q 11.1(\mathrm{QX} 1)$ & 20 & 30.0 & 1 & 0.03 \\
\hline $0.50 \mathrm{Q} 39.1(\mathrm{QC} 1)$ & 13 & 110.0 & 1 & 0.03 \\
\hline $2.13 \mathrm{Q} 38.3(\mathrm{QP})^{(c)}$ & 5.1 & 100.0 & 6 & 0.03 \\
\hline Standard Dipole & 23 & 262.3 & 18 & 0.03 \\
\hline Sextupoles & 20.7 & 25.0 & 4 & 1.0 \\
\hline
\end{tabular}

(a) Maximum nomprinciple field at $70 \%$ of full aperture.

(b) Includes one complete spare magnet.

(c) Two of this type may be used in place of $\mathrm{QC} 2$. 


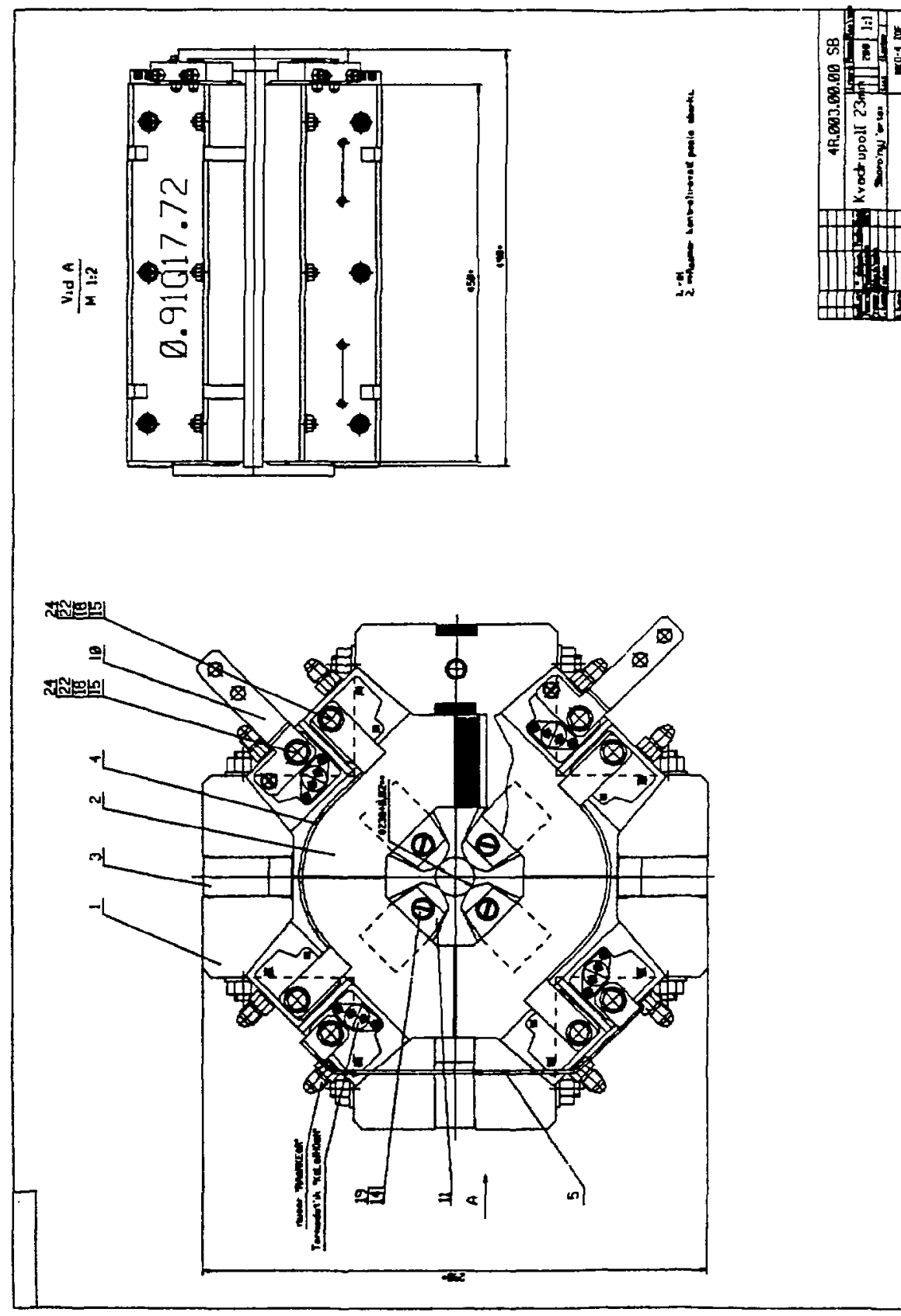

|II-1| 


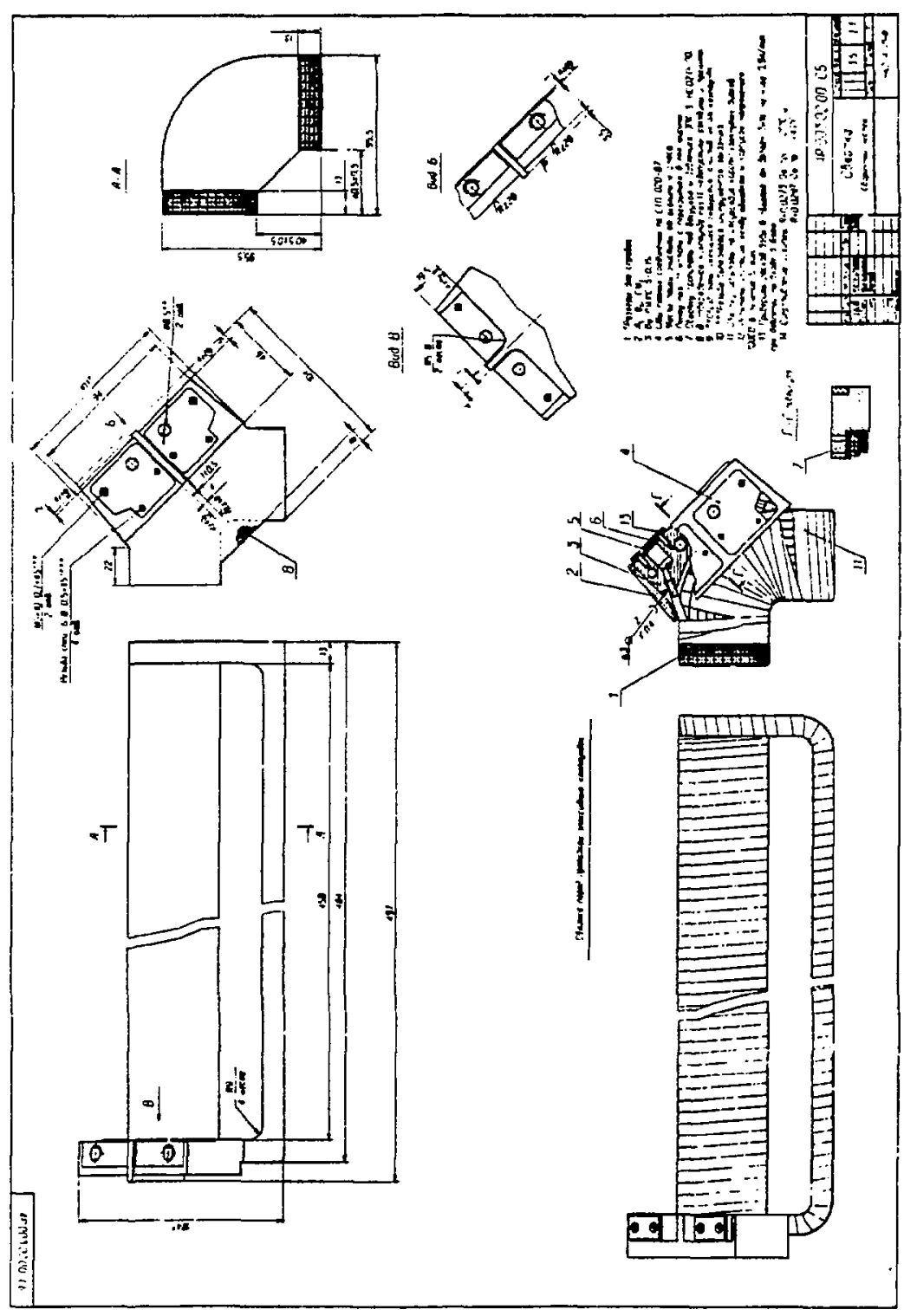

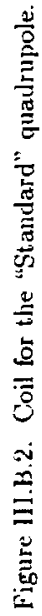




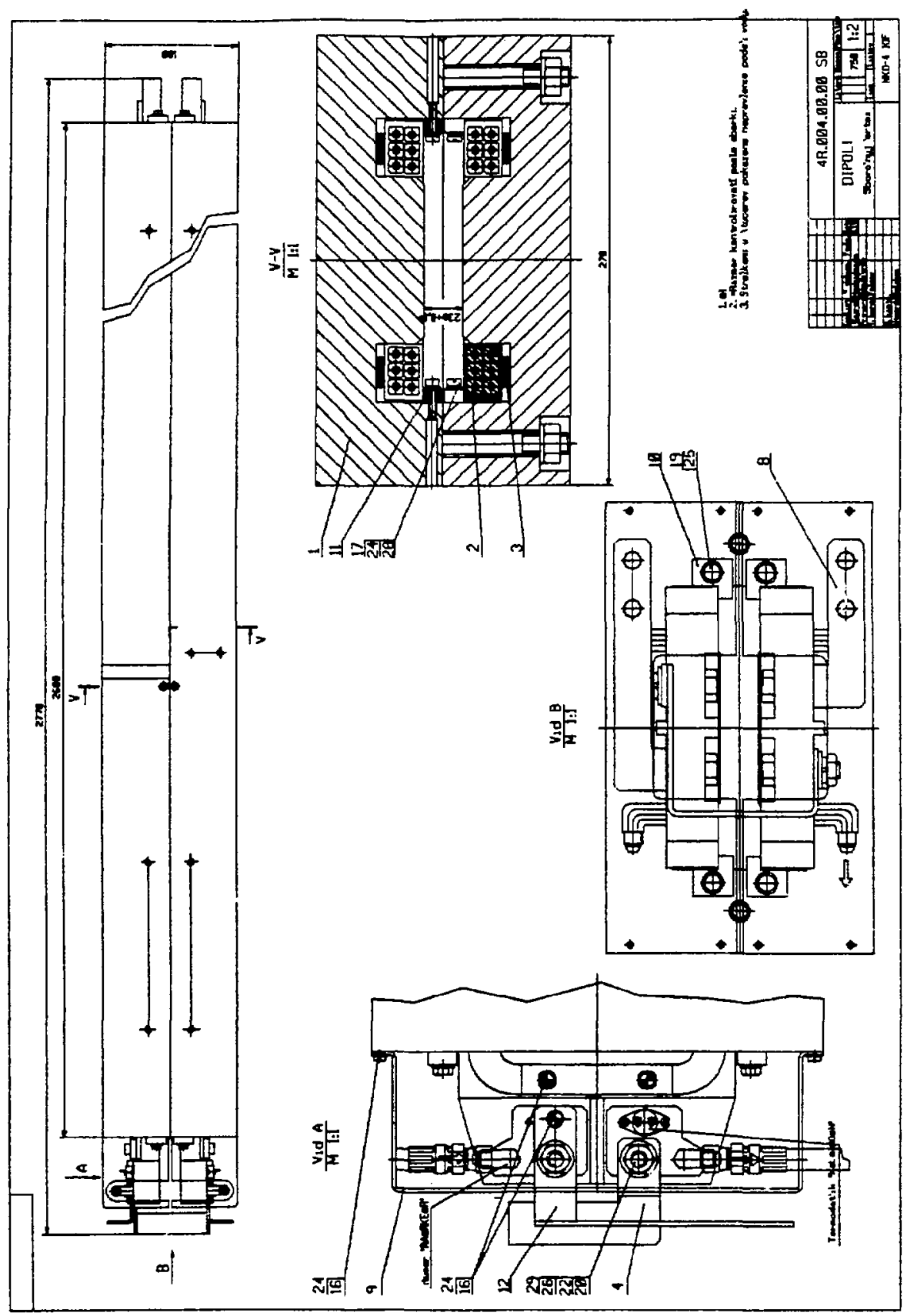

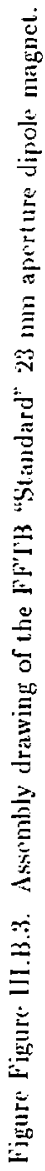

[I] - 1:3 


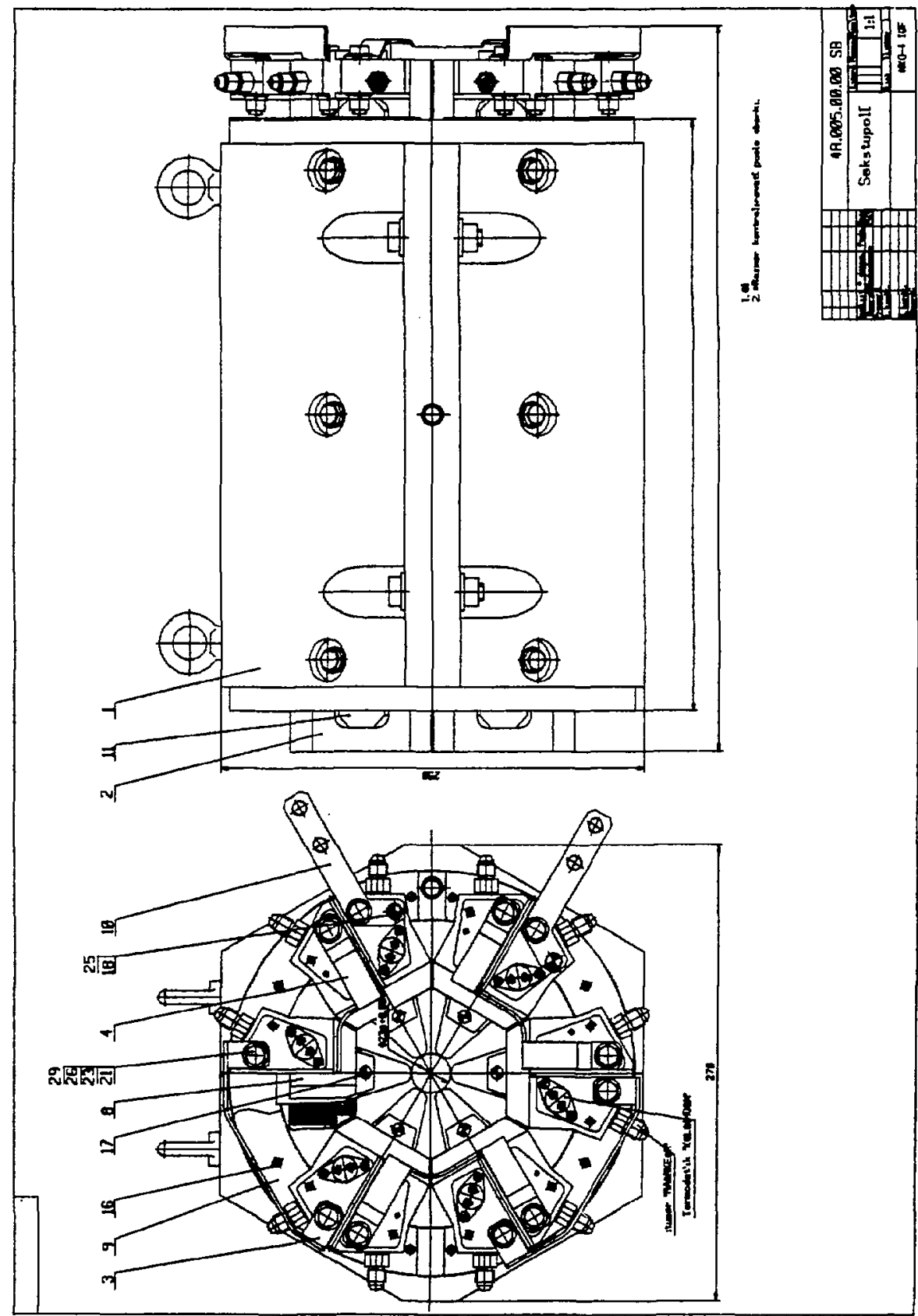

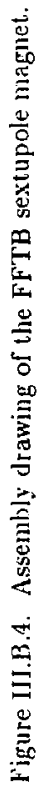

[1]-14] 
IV. Instrumentation 


\section{Instrumentation}

The beam-diagnostic instruments that will be used in the FFTB will be derived from devices already functioning in the SLC. This will include beam position monitors (BPMs), wire scanners, profile monitors, and 1.oroids, and a proposed beam size monitor.

\section{Bcam Position Monitors}

The beam position monitor's used in the FFTB will be copies of either the stripline monitors developed for the SLC ${ }^{1}$ Linac and Arcs, or will be based on the exposed-coax type used in the SLC Final Focus. Drawings of these devices are shown in Figure IV.l. A BPM will be inserted into the aperture of each quadrupole magnet. In order to maintain the inner diameter of the BPNs at more than ten times the expected beam size, the "strips" need to be moved into the space between the pole tivs of the magnets. This is done by extruding the bcan pipe into a four-leaf-clover cross section such that each BPM strip becomes the center conductor of a coaxial transmission line. Approximately $25 \%$ of the coax is exposed to the beam, so a sizable image charge flows on the center conductor. This generates a bipolar pulse at the end of the conductor whose amplitude is directly related to the intensity and position of the beam. The sum of all four amplitudes is proportional to the intensity alone, and is reasonably independent of position of the beam when it is within a few nillimeters of the center of the device.

The signal-processing electronics is similar to that used for the SLC ARC BPMs. A block diagram is show in Figure IV.2. The signal which emerges from the strips consists of two very narrow pulses of opposite polarity separated by twice the time it takes the beam to traverse the length of the strips-approximately $3 \mathrm{~ns}$. The signals are substantially wiclened by the time they pass through $200 \mathrm{ft}$ of coax with $2 \mathrm{Ghz}$ bandwidth. The first stage of the electronics is a low-pass filter which broadens the pulse to $10 \mathrm{~ns}$ or so. The signals are then digitized by a 14-bit ADC (13 bits per polarity). This compares to the 12-bit ADC used in SLC. The circuitry is being designed with a signal-to-noise specification of $1000: 1$, so the ADC granularity will not dominate the resolution of the system. The processing electronics resicles in CAMAC accessible to the FFTB data-acquisition network. See Section $\mathrm{IX}$ of this report for a discussion of the Control System for the FFTB. 
The arithmetic for calculating the bean centroid in the horizontal and vertical planes is given by:

$$
\begin{gathered}
I_{\text {bcam }}=T+B+N+S \\
X=k * \frac{(T-B)}{I_{\text {bcum }}}+X_{\text {offsct }} \\
Y=k * \frac{(N-S)}{I_{\text {beum }}}+J_{o \int f s c t}^{\prime}
\end{gathered}
$$

where, $T, B, N$, and $S$ identify the pulse height (less electronic pedestal) measured on the top, bottom, north and south strips of the BPM. The paraneters $X_{o f}$ set and $Y_{o f f s e t}$ are systematic offsets created by the mechanical and electrical properties of the BPM and cabling hardware. The constant $k$ converts the dimensionless ratio to displacement. typically millimeters. The value of $k$ can be derived analytically with reasonable accuracy. It is approximately equal to the radius of the strip from the centerline, and is a weak function of the displacement from the centerline. A constant value is sufficiently accurate for the purposes of the FFTB. Tle calibration fixture described in Section V of this report allows $k$ to be directly measured for each device.

There are two types of error involved in measurements of the beam position. The systematic errors dominate and are generated by clectrical imbalances caused by the: mechanical construction of the devices. attenuation differcuces between the four cables that bring the signals to the electronics, errors in the calibration of the signal-processing module, and mechanical offset errors of the whole derice. The sum of these errors will be held to less than 30 micjons on the average.

The second level of error is the pulse-to-pulse consistency of the measurement. These errors are dependent only on the noise in the signal-processing electronics and the least count of the ADC. Experience with the Final Focus BPMs in SLC' has shown that this pulse-to-pulse error can be below 2 microns. This will allow short term tracking of well-tuned beams. 
Experience with the SLC has also shown that the pulse-to-pulse relative resolution can be used to maintain good beam parameters on an hour-by-hour basis. The correlation plot in Figure IV.3 shows the horizontal coordinates from two neighboring BPMs in the SLC Final Focus. The range of the numbers demonstrates beam jitter and the narrowness of the plot shows the relative resolution of the two BPM -in this case less than 2 microns. Improved signal-to-noise, additional ADC bits, and smaller BPMs (smaller k) will yield even better relative resolution in the FPTB.

The calibration of BPMs used in the SLC has been done by measuring the electrical center of the BPM (i.e., where $T=B$ and $N=S$ ) relative to the mechanical center determined with a tooling surface. A new phitosophy is being adopted for the FFTB. Since the BPMs are placed inside the quadrupoles and it is ultimately desired to know where the beam is relative to the magnetic axis of the quadrupole it makes sense to measure BPM response when the beam is on the magnetic axis. Toward this end we have develojed a fixture which measures the axis of the field of the magnet, and simultaneously calibrates the response of the BPM. This is done by stretching a taut wire through the BPM after it is mounted in the quadrupole. If the wire is vibrated, then an $\epsilon m f$ will be induced across the ends of the wire if there is a net integral magnetic field along its length. The symmetry axis of the magnet can be found by moving the end of the wire in micron steps until the signal is minimized. The vibration can then be stopped and a voltage pulsed sent down the wire to simulate the passage of the bean. This provides a measure of the response of the BPM to a beam that passes at that wire position. The software can then use this BPM response as its reference for locating the beam relative to the axis of the magnet. An additional benefit of this method is that the BPM is calibrated in the quadrupole where it will stay when installed. This eliminates the adcled uncertainty introduced if a tooling fixture is used in internediate calibration steps. The calibration facility is further discussed in Section $V$ of this report. 


\section{Wire Scanners}

Wire scanners used in FFTB will be of the same design used in SLC. 'These consist of a 45-degree actuator capable of being moved in sub-micron steps across the beam. By moving one of three wires across the beam one can measure the horizontal and vertical profiles of the beam as well as a 45 -degree projection. The apparatus is shown in Figure IV.4.

The intensity of the beam striking the wire is observed with a phototube detector placed at 90 degrees from the beam. The signal amplitude is digitized by an ADC in C.AMAC attached to a MICRO. A typical sweep of the SLC beam is show in Figure IV.5.

\section{Profile Monitors}

Profile monitors based on luminescent screens have been in use at SLAC for many years. They are useful for getting a visual reading on the profile of the beam. They are. however, only useful at places in the optics where the bean is larger than $0.5 \mathrm{~mm}$ or so. as their resolution is limited by the grain size of the screen.

The devices consist of an insertable screen and a vacuum window observed by a wellshielded video camera. The video image can also be digitized for computer processing. There has been limited success with emittance measurements of beams in the SLC using digitized images. The primary benefit has cone fr - . le realtime visualization of the beam and its background tails.

\section{Toroids}

Toroids used in the Personnel Protection System (Section $X$ of this report) will also be used as beam transmission monitors. Thesr are useful for monitoring beam loss and to provide a calibration of the overall gains of the BPM electronics. 


\section{Stoppers and Beam Dump}

Stoppers will be utilized for both personnel protection and machine protection during parameter studies. A bean stop placed just downstream of the initial $\beta$-matching section of the FFTB, for example, will allow the incoming beam phase space to be matched to the remainder of the lattice before it is permitted to pass through the chromatic-correction sections and on to the focal point.

The final beam dump is made of aluminum and is water cooled. The sump is capable of absorbing $70 \mathrm{~kW}$ for a short period without suffering mechanical damage. Under normal operating conditions the beam power is limited to $1 \mathrm{~kW}$, but this dump will be capable of hanáling the full power of a single SLC beam in the case that such a beam is accidentally transmitted through the FFTB.

\section{Beam Size Monitors}

Conventional profile monitors would not work to measure the transverse bunch dimensions at the focus wheie they range from $1 \mu \mathrm{m}$ down to $60 \mathrm{~nm}$. A detailed description of the monitor to be built for the FFTB is given in Ref. 2 .

lons are created by the passage of an electron bunch in a pulsed gas target at the focus. Essentially, the information on the transverse dinensions is obtained through the kick givon to the ions by the space charge electric field of the bunch. After passage of the bunch, the ions are emitted in the transverse plane and are collected by an ion detector.

ln the case of lieavy argon ions that move only slightly during the bunch passage, their maximum velocity is proportional to the maximum electric field. The latter is inversely proportional to the largest transverse beam climension, namely the horizontal dimension. The measurement of the minimum time-of-flight of the ions from the focus to the detector will give this horizontal dimension (see Figure IV.6).

In the case of light helium ions that are trapped by the electron bunch and oscillate in its potential well, their oscillation amplitude depends on their initial position. In a horizontally flat bunch, the horizontal amplitude and the final horizontal ion velocity are. on an average, larger than the vertical ones. The angular distribution is peaked 
along the horizontal direction of the transverse plane (see Figure IV.7). On the contrary: the angular distribution is isotropic lor a round beam. The measurement of the angular distribution will give the bunch aspect ratio. It will also be used to correct the measurement of the horizontal dimension with heavy ions that have a relatively weak dependence on the aspect ratio. This measurement requires the lFETB to be operated with electrons only.

It is worth noting that these measurements are not biased by any beam jitter. This also means that they cannot give any useful inlormation on the beam position.

The proposed setup of the Beam Size Monitor is made of a pulsed gas target and an array of ion detectors. Only the princjple of the experimental setup is given here.

To iuject a pulse of gas (about $100 \mu$ s long). a smali hole $(0.4 \mathrm{~mm}$ in dianeter) the wall of the pipe is opened by a shut ter just before the bunch passage. In a molecular segime. the structure of the gas jet is rapidly destroyed by rellections on the walls of the beam pjpe ( $t \mathrm{~cm}$ in diameter). One has more a gas target--filling the pipe section and extending a lew centimcters longitudinally-than a gas jet.

A simulation, assuming diffusive reflection and negligible attachment on the walls. shows that the gas density at the focus is maximum $25 \mu$ salter the shutter closing. It reaches $2 \times 10^{12}$ argon atoms per $\mathrm{cm}^{3}$ for an inlet pressure of 2 Torr. The gas target extends longitudinal: over $2.5 \mathrm{~cm}$ FWHM and over $\pm 4.5 \mathrm{~cm}$ total at that time.

A second hole allows injection of pulses of helium. As the latter has a larger mean velocity, it diffuses through the hole laster than argon does. A larger gas density at the focus $\left(4 \times 10^{12}\right)$ can be reached with the same inlet pressure and hole cross section. The maximum density is reached at the closing of the shutter and extencls longitudinally over $3 \mathrm{~cm}$ FWHM and over $\pm 74 \mathrm{~cm}$ total.

The pulse of gas is pumped before the passage of the next bunch (100 ms later) through the beam pipe. by two pumps ( $50 \mathrm{l} / \mathrm{s}$ at $10^{-6}$ Torr) connected to $25 \mathrm{~cm}$ upstream and downstream of the focus. The level of the resiclual pressure will be reached in a few milliseconds. 
The ion detector consists of an array of eight pairs of microchannel plates (MCP) surrounding the beam focus at a distance of $6 \mathrm{~cm}$ (see Figure IV.T). Before reaching the MCPs, the ions will pass through a narrow slit in a Wolfram shielding $2 \mathrm{~cm}$ thick.

A small potential difference $(=100 \mathrm{~V})$ across the slit in its second part gives a longitudinal kick to the argon ions. The longitudinal displacement $z$ (a few millimeters) of the ion impact on a MCP is proportional to its charge $Z$ and inversely proportional to its kinetic energy. The simultaneous measurement of $z$ and of the time-of-flight for cach ion allows us to discriminate between argon ions of different charges.

The NCPs have a rectangular shape. $40 \times 50 \times 0.5 \mathrm{~mm}$, with a hole diameter of $12 \mu \mathrm{m}$. The gain reaches about $3 \times 10^{3}$ at $1 \mathrm{kV}$. A pair of MCP insures an overall gain of $10^{\top}$.

Each pair of MCPs is read by nine parallel collectors (anode strips), as shown in Figure IV.9. The relatively large number of strips ( 72$)$ is chosen to reduce the pileup of signals, allowing fast measurements of the beam size. They give an angular resolution of 87 mrad. The number of strips is increased near the horizontal direction, where the counting rate is peaked for flat beams and where one needs higher angular resolution.

To measure the longitudinal position of the ion impact, each anode strip is of a resistive type $(1 \mathrm{k} \Omega$ ). allowing a charge division for this measurement. A resolution of $0.4 \mathrm{~nm}$ is expected.

Signals collected on each side of the strips are anplified and analyzed in time and amplitude by Time Digital Converters (TDC) and fast Amplitude Digital Converters (ADC), respectively. The comparison in amplitude of the two signals from one strip will determine the charge division between them. and will give the longitudinal position $z$ of the ion impact.

The ion counting rate can be estimated from the gas target density and the ionization cross section. For a gas density of $10^{12}$ atoms per $\mathrm{cm}^{3}$ (i.e., a pressure $P=6.310^{-4}$ Torr) and a $2.0 \mathrm{Mb}$ cross sertion. $2000 \mathrm{Ar}^{+}$ions are created per millimeter along the beam. Considering a $\pm 200 \mu \mathrm{m}$ target length and a $20 \%$ detection efficiency, about 150 ions would be detected per burst. enough to measure the beam radius with less than ten bursts. 
Figure IV.10 shows the time-of-flight spectrum that can be obtained with 500 jons according to a simulation code.

The helium ionization cross section is about 7.5 times smaller than the argon cross section. One would need to increase the gas density for a measurenent in a few bursts. The production of $\mathrm{He}^{2+}$ is negligible and there is no need to separate it out.

Finally, the $\mathrm{He}^{+}$time-of-flight is much smaller than the $\mathrm{Ar}^{+}$one, as its mass is ten times smaller. The fastest $\mathrm{He}^{+}$will arrive $60 \mathrm{~ns}$ after the bunch passage for a $6 \mathrm{~cm}$ flight path, and in the case of flat beam, $R=16$.

The mode of operation could be first a few electron bursts witl a pulse of argon gas, followed by several others with pulses of helium gas. 


\section{References}

1. J. C. Denard, G. Oxoby, J. L. Pellegrin, and S. Williams, "Monitoring of the Stanford Linac Microbunches," SLAC-PUB-305S (1983), Proceedings of the Particle Accelerator Conference, Santa Fe, New Mexico, 1983.

2. J. Buon et al., "A Beam Size Monitor for the Final Focus Test Beam," LAL/RT/90-1: (1990). 
(a)

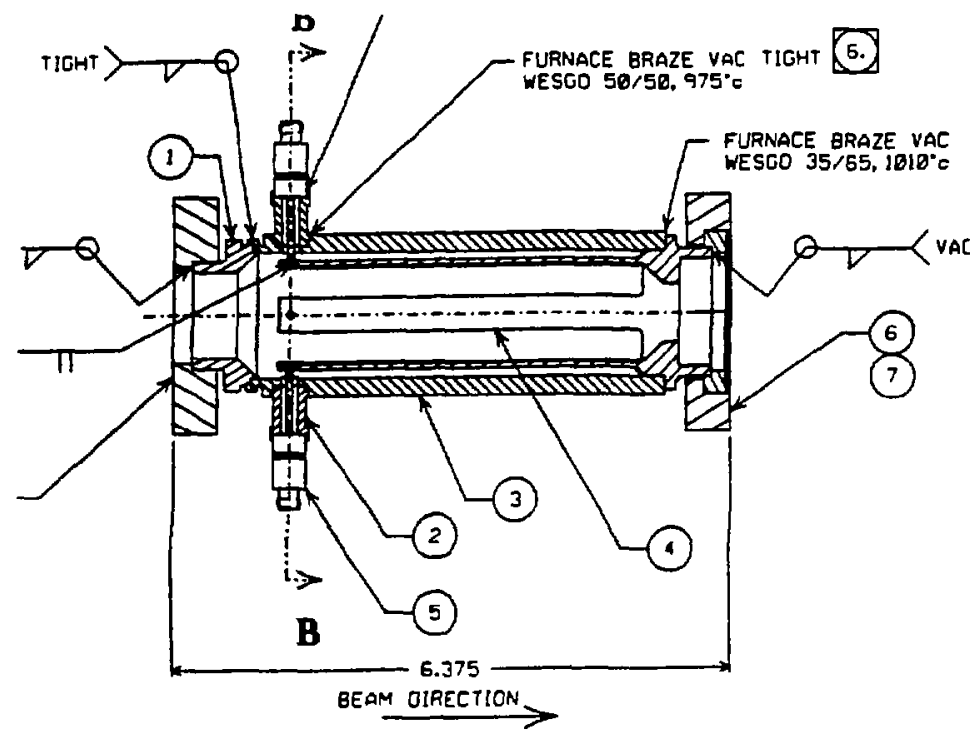

(b)
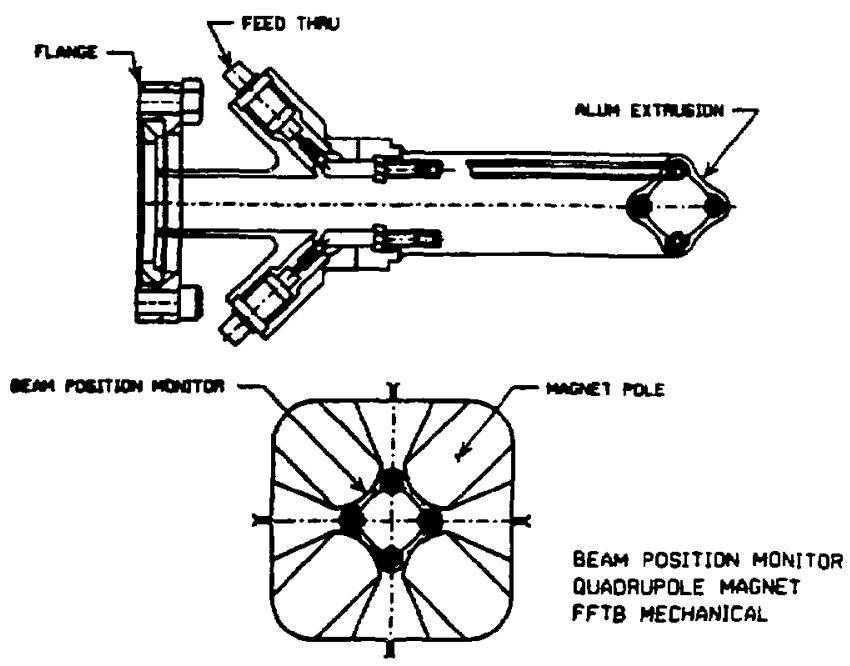

Figure IV.1. (a) Assembly drawing of a stripline BPM used in the SLC. (b) Assembly drawing of an exposed-coax BPM for the FFTB. 


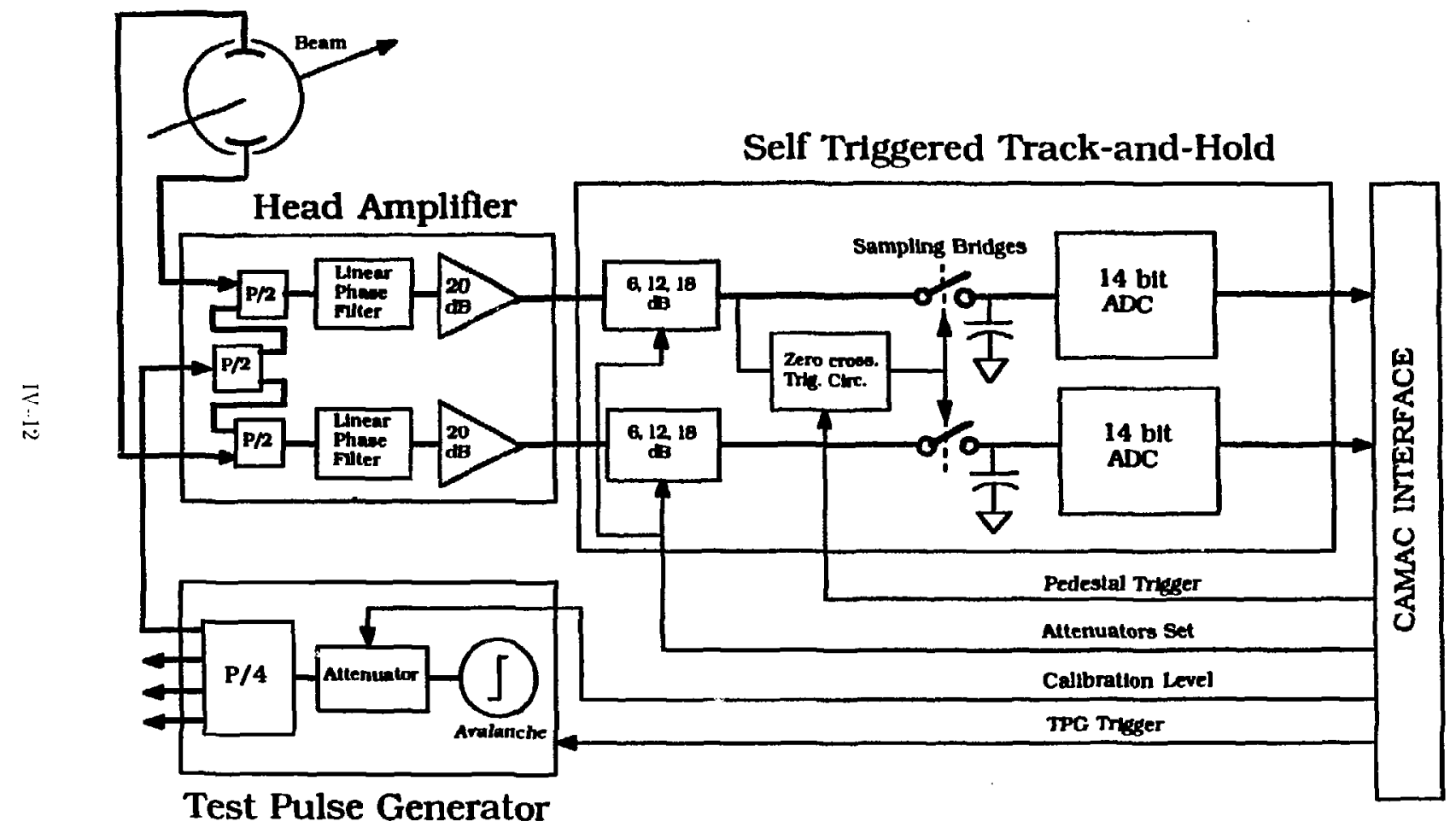

Figure IV.2. Schematic of the signal-processing electronics used to make beam position measurements. 


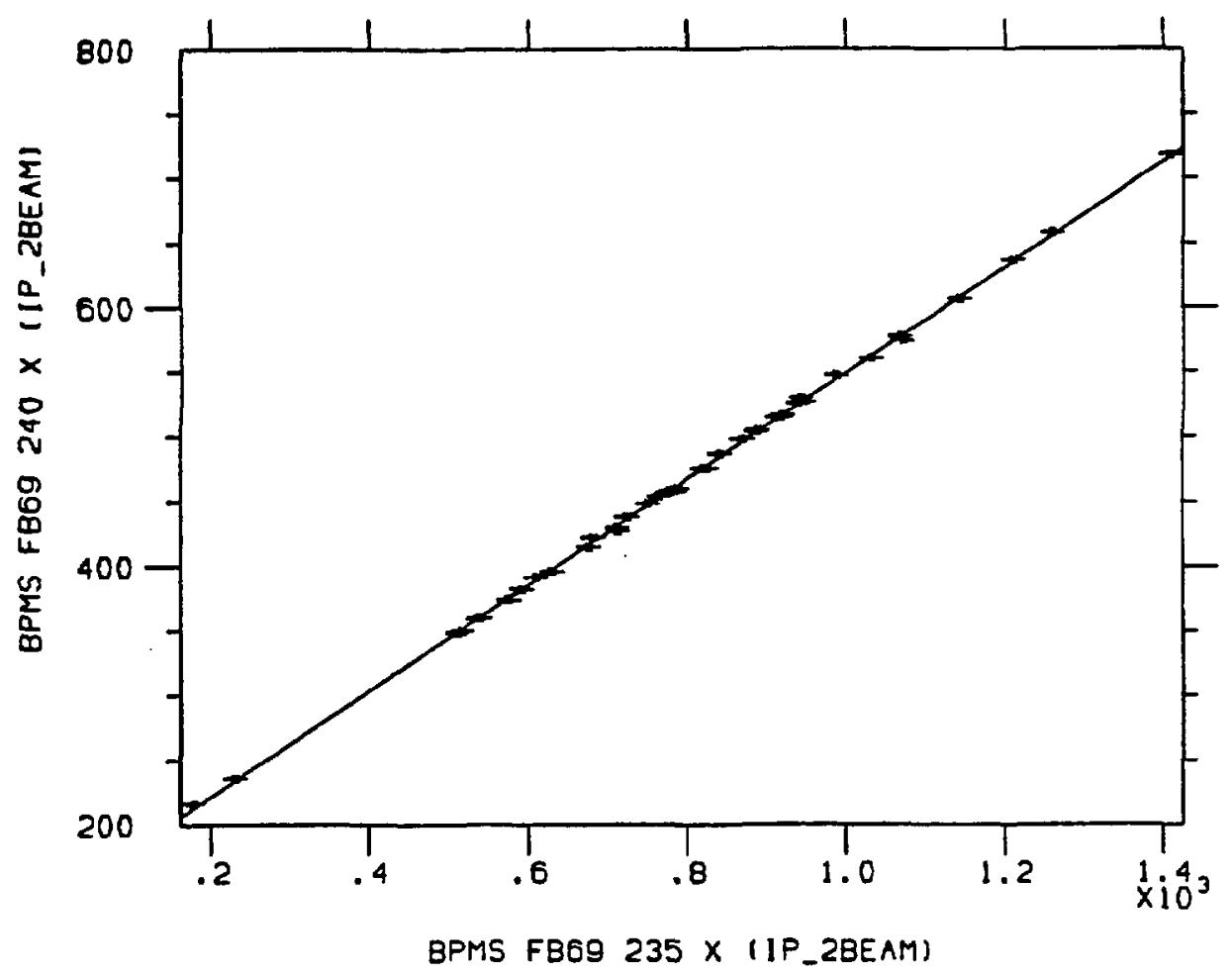

Figure IV.3. Correlation plot of measurements of the horizontal beam position made by two different exposed-coax BPMs in the SLC Final Focus quadrupole triplet. 


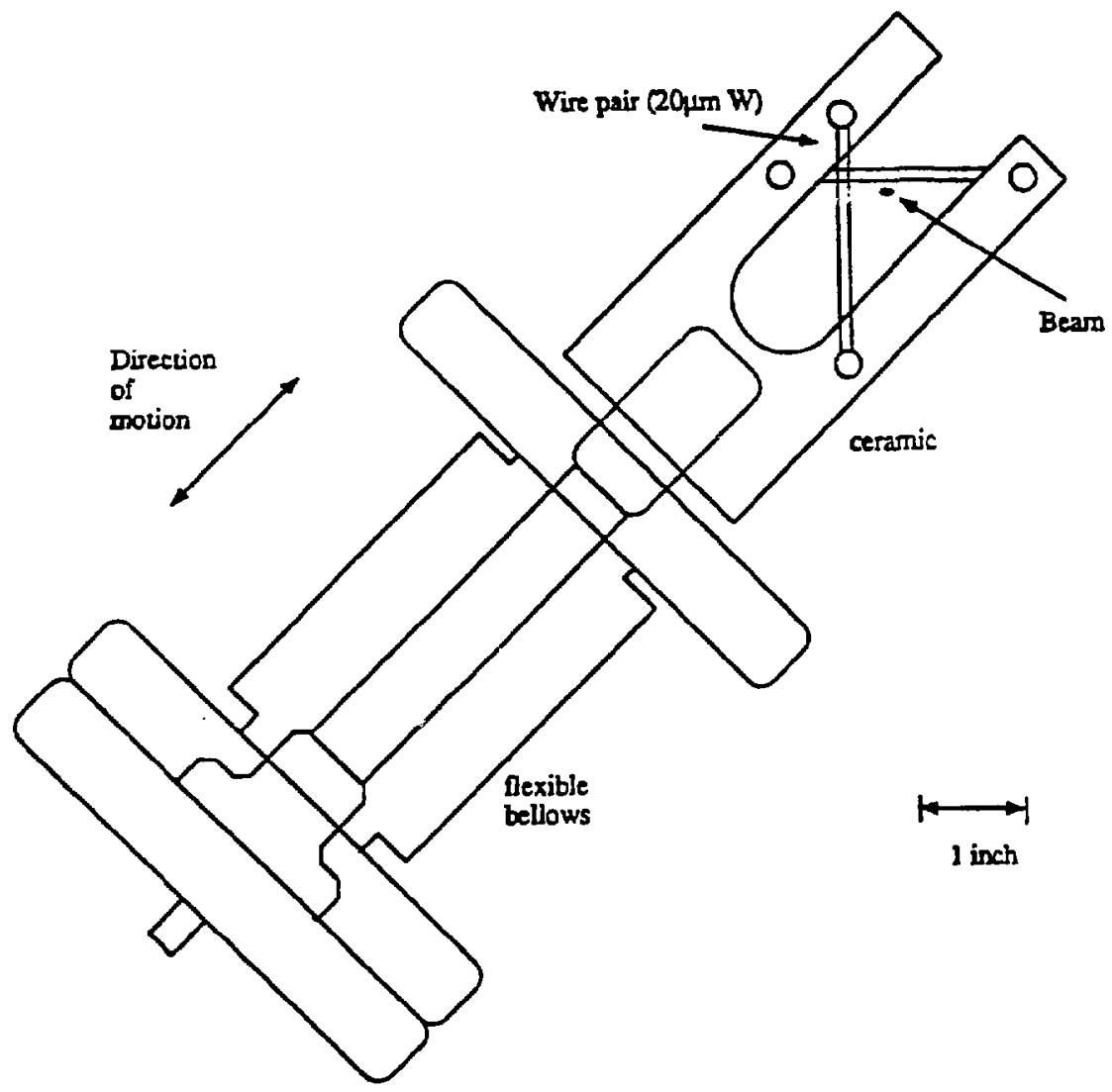

Figure IV.A. Schematic drawing of a wire scanner used in the SLC. 


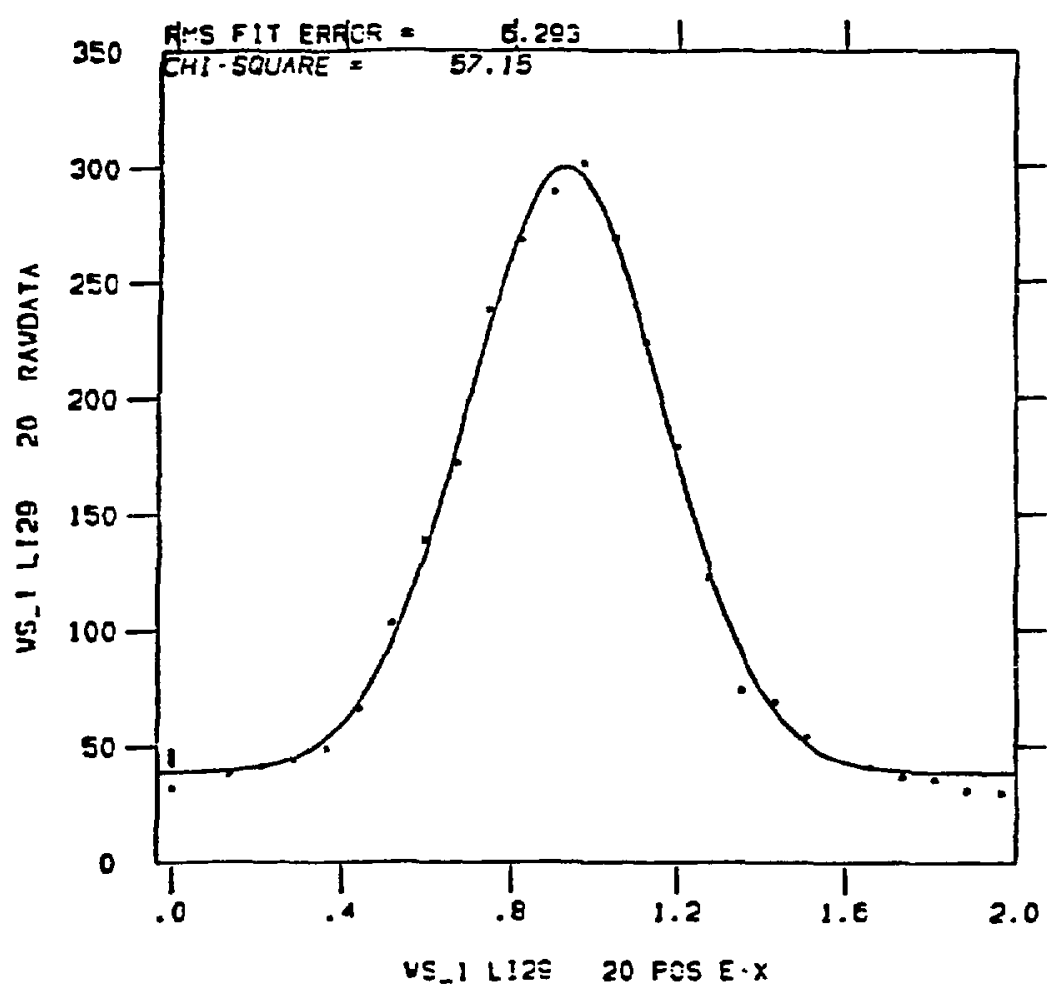

Figure IV.5. Profile of the SLC electron beam as measured with a wire scanner at. the end of the linac. Such data will also be avalable and useful during operation of the FFTB. 


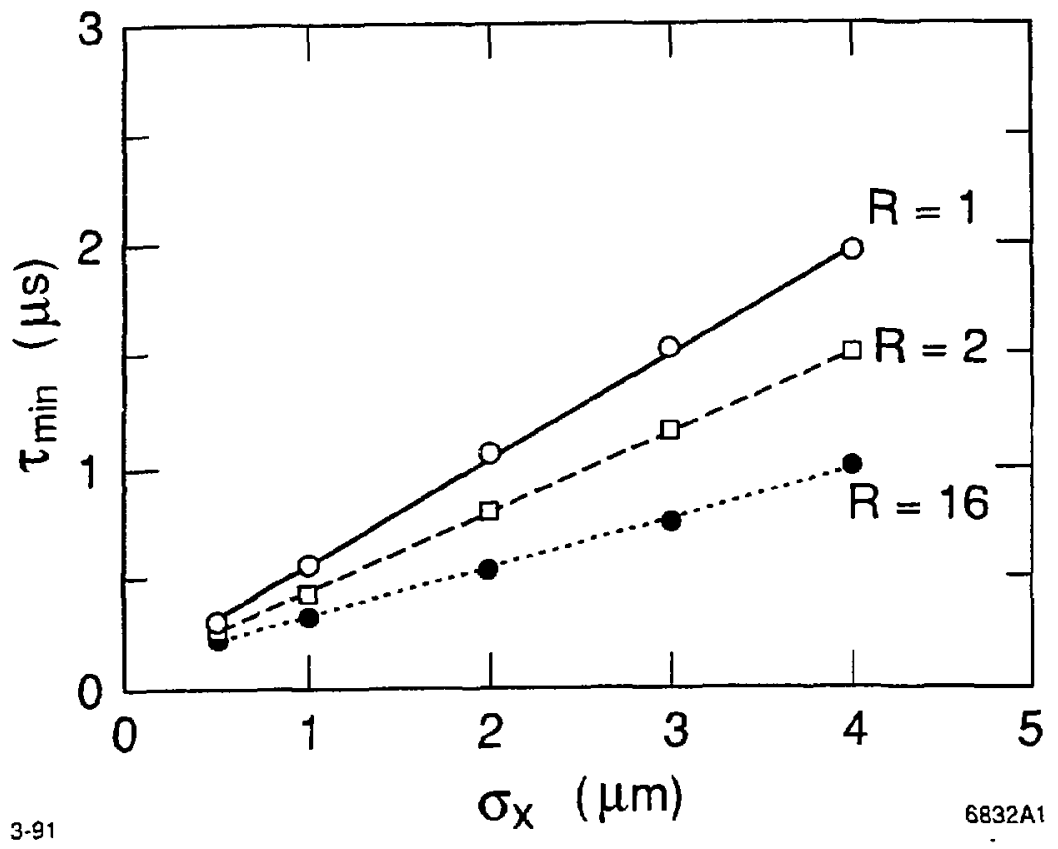

Figure IV.6. Varjation of the minimum $\mathrm{Ar}^{+}$tine-of-flight $\tau_{\min }$ for a $6 \mathrm{~cm}$ flight path as a function of the horizontal bunch dimension $\sigma_{x}$ for different aspect ratios $R=\sigma_{x} / \sigma_{z}$ $\left(\sigma_{z}=0.5 \mathrm{~mm}\right.$ and $\left.N_{e}=10^{10}\right)$. 


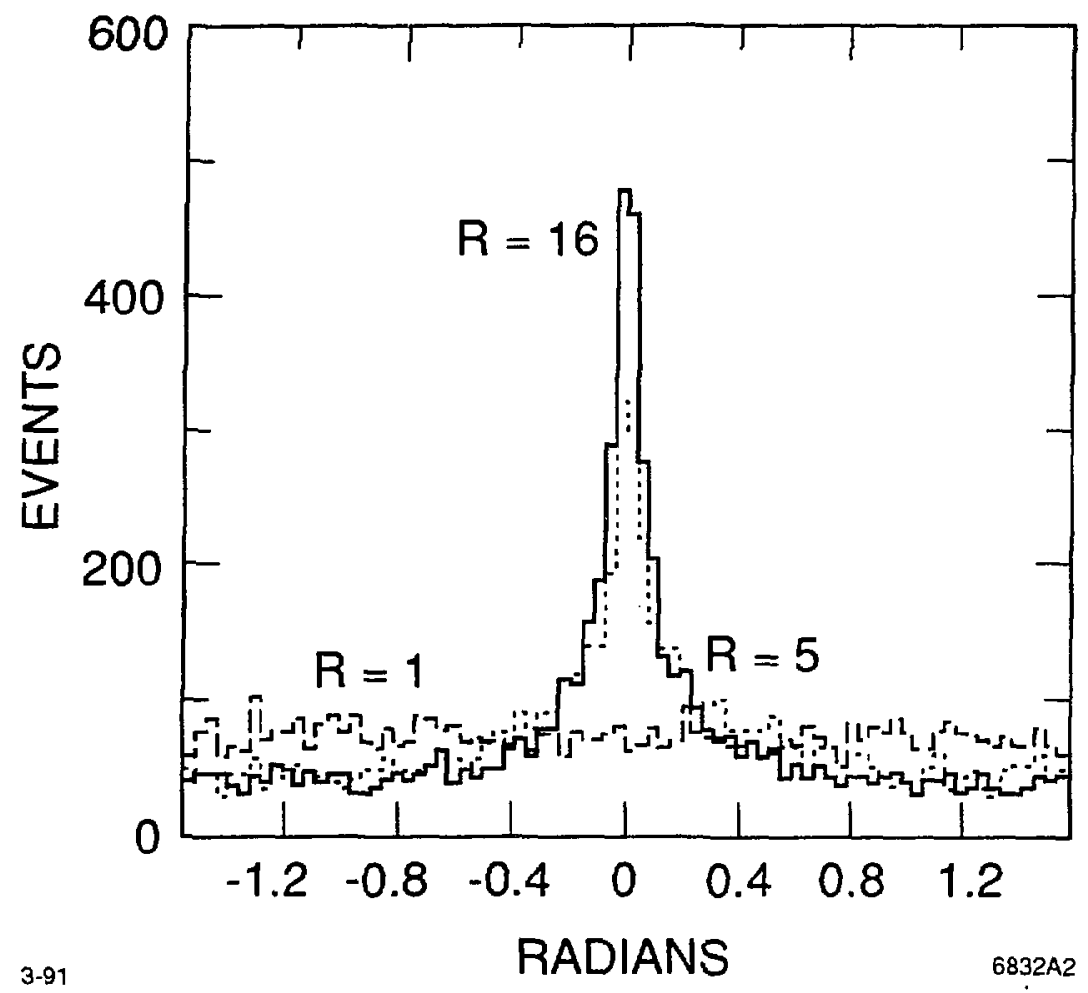

Figure IV.7. A simulated distribution of $\mathrm{He}^{+}$ions for three different beam aspect rations $R$ (athef angle 0 corresponds to the hoizontal direction). The beam dimenstions are: $\sigma_{x}=1 \mu \mathrm{m}, \sigma_{z}=0.5 \mathrm{~mm}$, and $\left.N_{e}=10^{10}\right)$. 

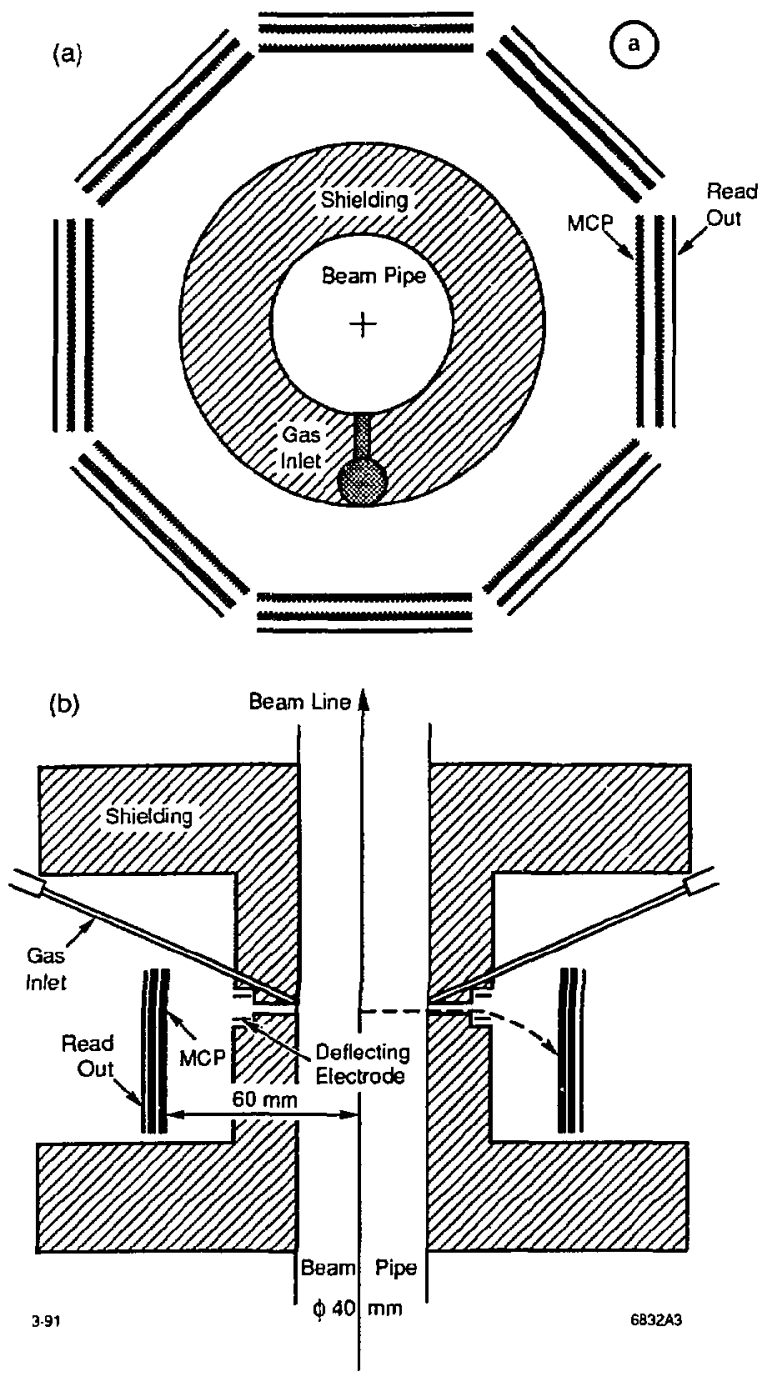

Figure IV.8. Schematic view of the ion detector: (a) Transverse section at the FFTB focus. (b) Longitudinal section along the beam line. 


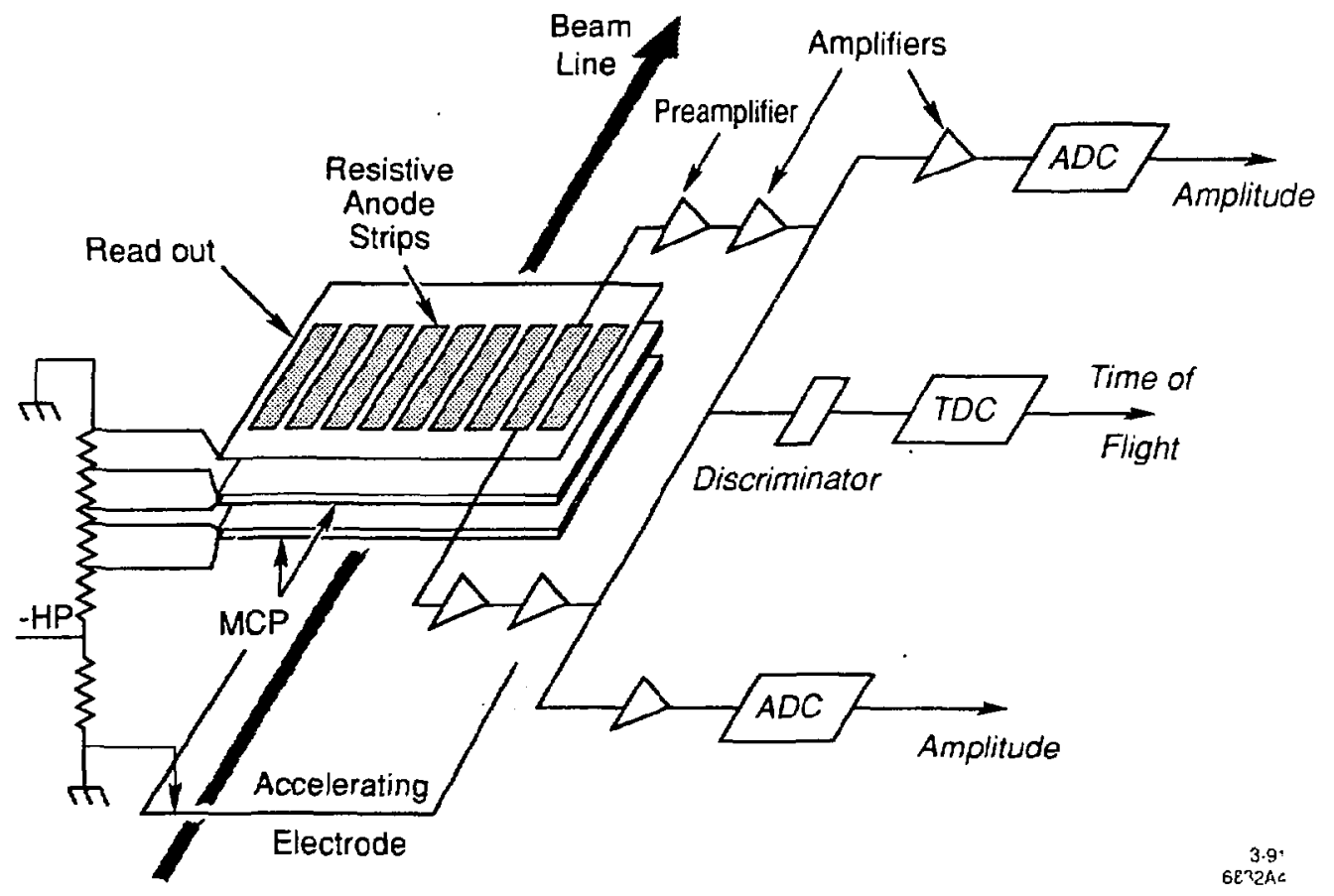

Figure IV.9. Schematic view of an MCP pair with the anode sips and the associateri electronics. 


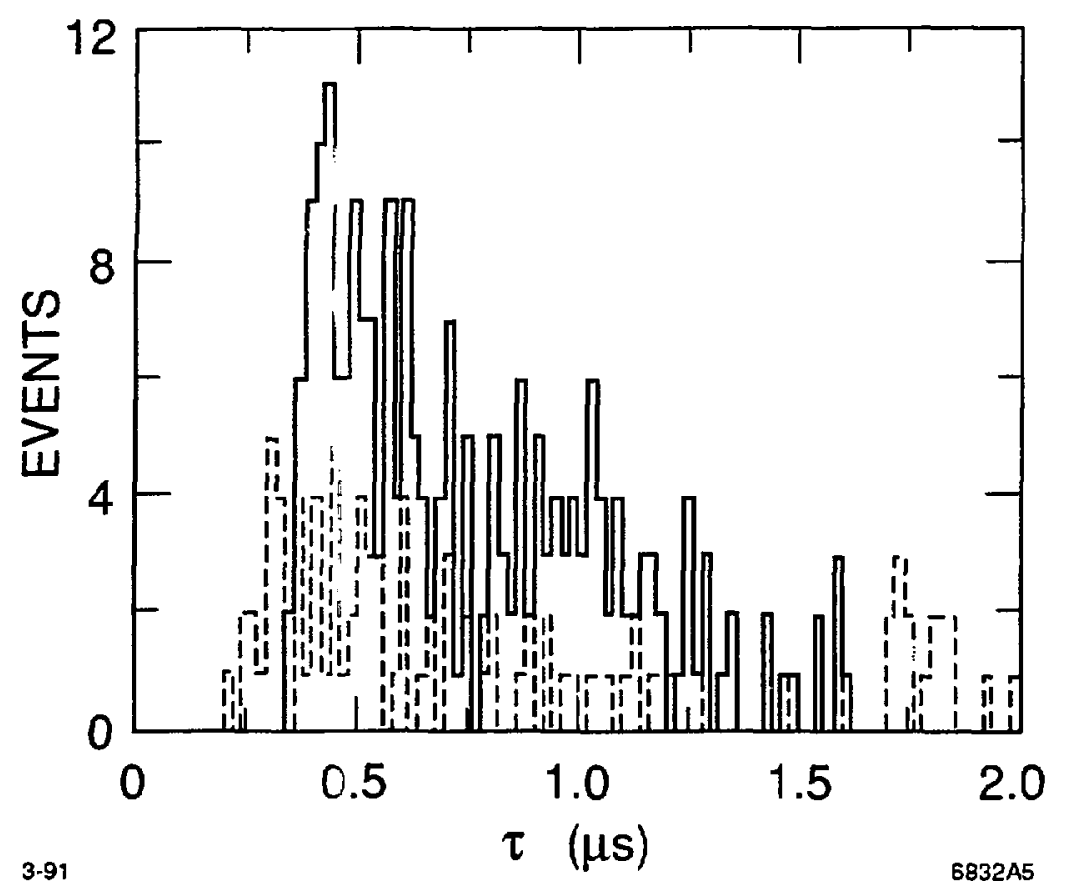

Figure IV.10. A simulated time- f-flight spectrum of $500 \mathrm{Ar}^{+}$ions produced by an electron beam $\left(\sigma_{x}=1 \mu \mathrm{m}, \sigma_{y}=. \mu \mathrm{m}, \sigma_{z}=0.5 \mathrm{~mm}\right.$, and $\left.N e=10^{10}\right)$ taling account al the experierl experimental lesintution. 
V. Magnetic Measurement and BPM Calibration 


\section{Magnetic Measurement and BPM Calibration}

An entirely new calibration facility has been developed for FFTB magnet and BPM calibration and is shown in Figure V.1. In the past, magnetic fields were measured independently of BPM calibration and in separate fixtures which necessitate disassembly of the guadrupole and insertion of the BPM AFTER each was separately calibrated. These measurements were made with respect to the mechanical axis of the devi.es. We now hare developed a method to make the measurements with respect to the magnetic axis, which is the important axis for bean optics. This new fixture enables us to measure both the magnetic axis of the field and the BPM response in a single fixture in such a way that it is no longer necessary $($ ) disassemble them before final installation.

The calibration fixture is mounted on a seismically isolated table. The method is accomplished by stretcining a taut wire through the assembled quad and BPN. The lightweight wire is tensioned with abcut 100 grams and made to vibrate at a frequency in the range of $50 \mathrm{~Hz}$. The ends of the wire are held by two precision stages which can be moved in two dimensions in sub-micron steps. In order to locate the magnetic axis of the energized quadrupole the wire ic vibrated by an audio speaker's voice coil which is mechanically attached to the wire. The $\mathrm{emf}$ generated across the wire can be measured and minimized by moving the stages holding the ends of the wires. Figure V.2 shows the generated emf as a function of the displacement of one end of the wire.

The vacuum flanges on each end of the BPM will be the tooling surfaces to which the wire location will be referenced. This is accomplished by the device shown in Figure V.3. A ring gauge is mounted on the BPM fiducial flange located by three pads (one is spring loaded). It can be rotated in a plane perpendicular to the quadrupole's axis. A micrometer is mounted on the ring gauge and run in until it barely touches the wire; this can be detected electrically. This is repeated after rotating the gauge 180 degrees. The average of the two positions gives the location of the wire relative to the flange tooling surfaces. 
Once the magnetic axis is found with the vibrating wire, the vibrations are stopped and a voltage pulse that simulates the beam is sent down the wire from the upstream side. Average voltages are read out from the track-and-hold circuitry applied to each strip of the BPM. One set of electronics will be switched from one strip to the next using a coaxial switch. This will eliminate need to calibrate four sets of electronics. These four voltage readings will be used in the FFTB feedback system to relate beam positions to the magnetic axis measured in this apparatus.

The effects of wire sag will be minimal. A 2-meter wire stretched with 100 grams will sag about 25 microns from center to end. However, the ring gauge will locate the wire at either end of the BPM/quad-a distance of about 0.5 meter. Over this distance the sag is about 5 microns. Further, since the sag affects both the magnetic axis measurement and the BPM measurement, the effect of sag tends to cancel.

We expect to calibrate the BPMS used in the FFTB quadrupoles, and to determine the null axis of the quadrupole fields well enough to center the two to within 30 microns of each other. In addition, we also will use the apparatus to measure the excitation functions of all magnets used in the beamline, and to check the harmonic properties of their fields. 


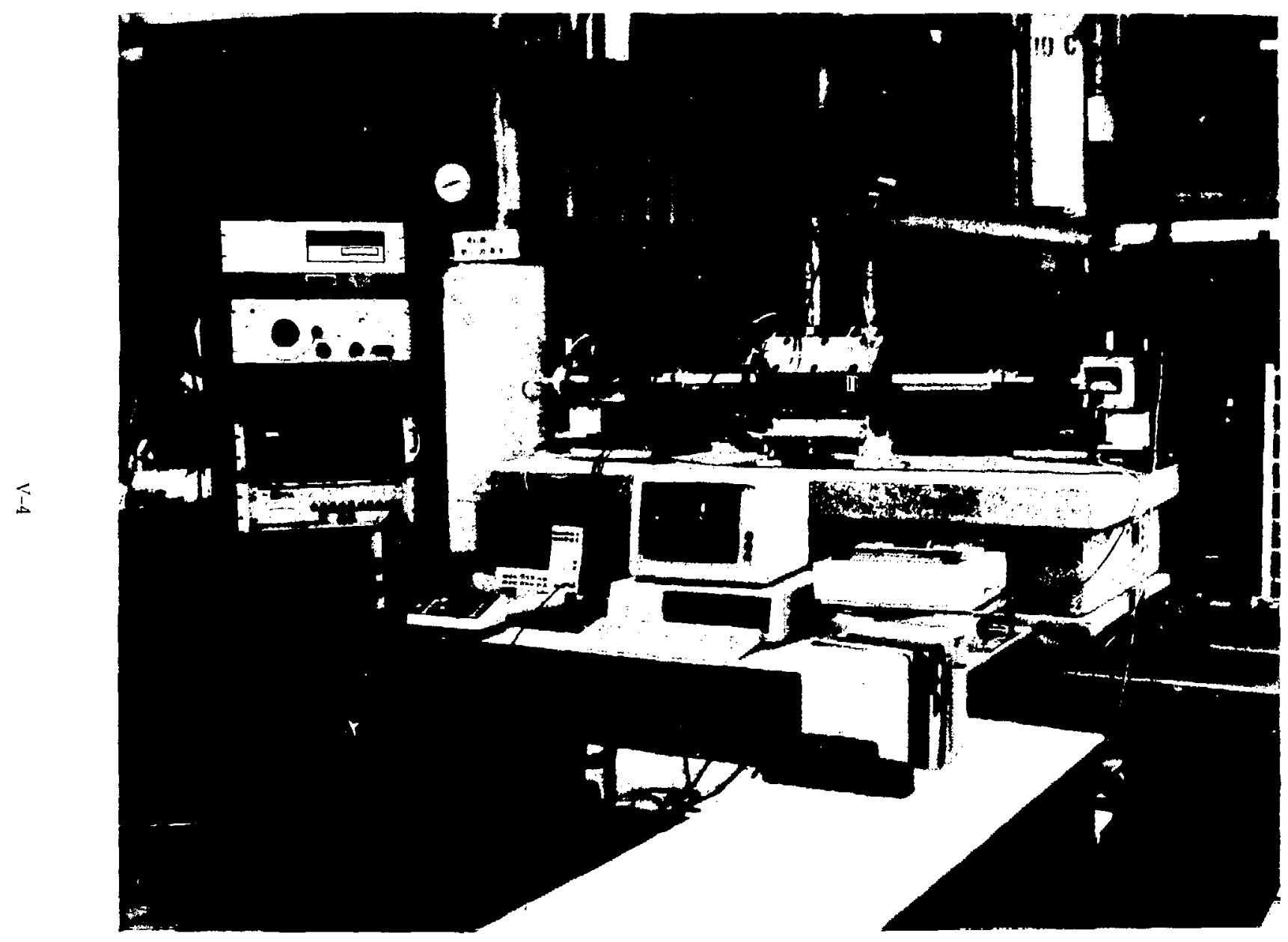

Figure V.1. Photograph of the BPM/Quadrupole calibration facility. 


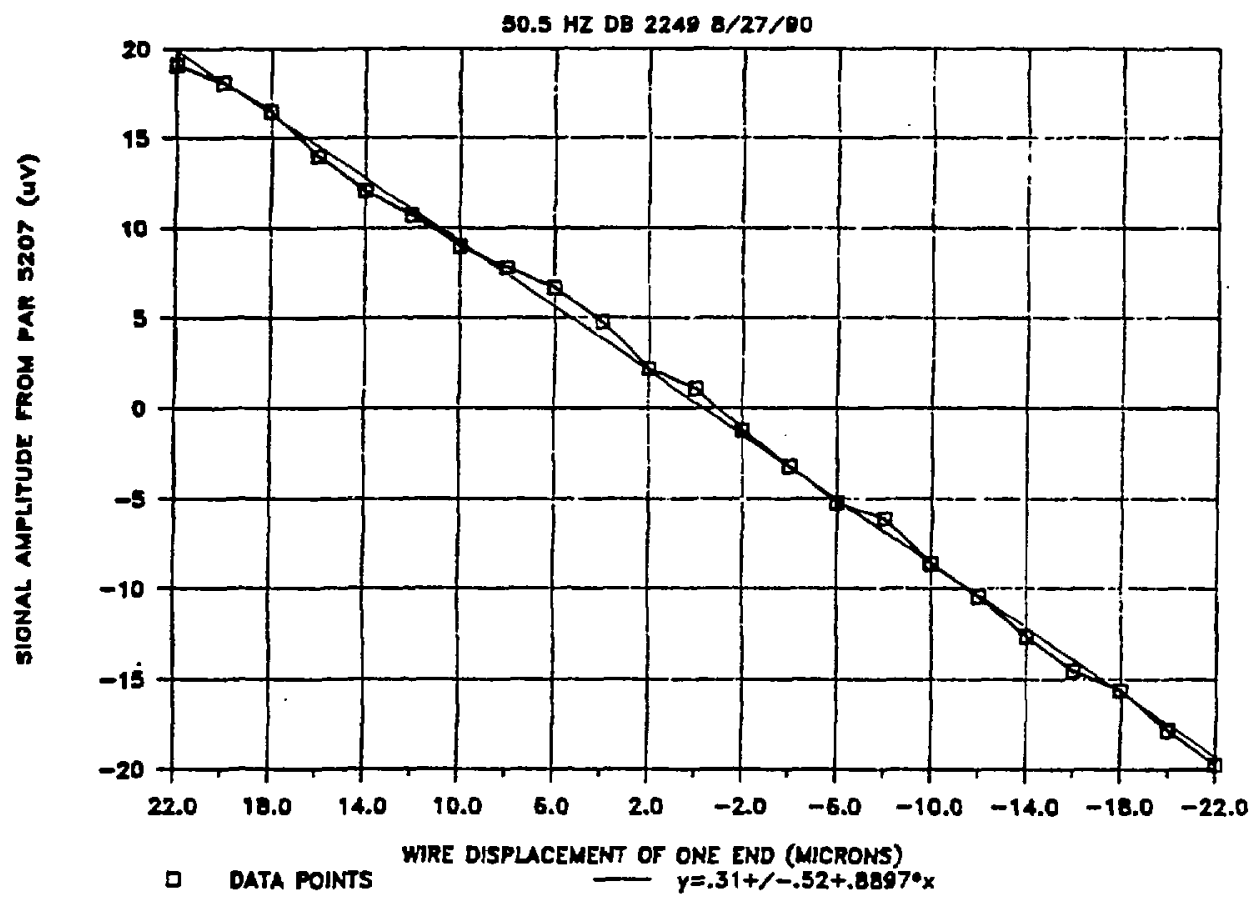

Figure V.2. Example of the emf induced on the vibrating wire as the position of the wire is scanned through the aperture of a quadiupole maguet. 


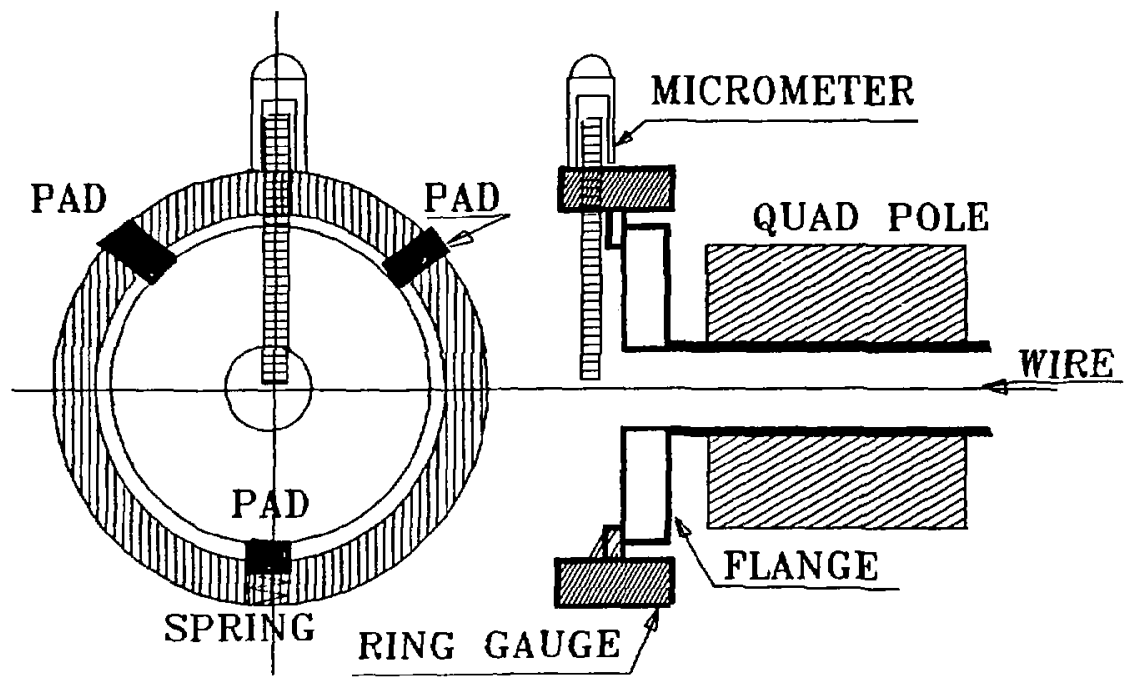

Figure V.3. Conceptual schematic of the tooling fixture used to locate the position of the vibrating wire. 


\title{
VI. Mechanical Alignment
}

\author{
and Stabilization
}




\section{Mechanical Alignment and Stabilization}

\section{VI.A Introduction}

Errors in the positioning or orientation of the magnetic elements of the FFTB (with respect to the ideal beam line coordinates) degrade the performance of the system by introducing anomalous dispersion and/or coupling into the beam phase space. These errors can occur as misplacements in the initial settings of the beam line elements, or as motions of the elements after their installation. Detection and correction of alignment errors must be done either by additional alignment hardware designed specifically for this purpose, or by deduction from on-line measurements of the beam orbit and phase space at various points along the beam line.

The relative alignment of the beam line will suffer from finite motions that occur with a range of frequencies from $\approx 100 \mathrm{~Hz}$ down to the static limit. Alignment errors that are within certain tolerances (typically a few tens of microns for the FFTB) can, in principle, be compensated by tuning the parameters of the lattice. This method will be successful if the errors are static, or at least develop slowly compared to the time required to accomplish the tuning. To completely tune the lattice may require several hours. Motions of beam line elements that produce microns of displacement in shorter times must be monitored and elininated by additional hardware. With a few exceptions, "vibrations" that occur at frequencies above the bandpass of the alignment hardware can not be corrected in any fashion. In some cases it may be possible to observe and accurately determine motions from neasurement of beam parameters. These can be corrected electrically by adjusting the strengths of trim magnets, but this technique will fail at frequencies above one sixth of the repetition rate of the machine. Fortunately, propagation of acoustical waves with frequencies above a few tens of Hertz is significantly damped in materials such as concrete and earth. With proper care in design, it is possible to isolate the beam line elements so that motions at these frequencies are negligible.

In this section of this report, we discuss the hardware and techniques that will be used to align and monitor the positions of the elements of the FFTB. Data will also be presented from studies of the stability of the ground and structures on which the FFTB 
will be constructed. Tuning and alignment strategies that are based on measurements of the beam properties are discussed in the FFTB Optical Handbook.

\section{VI.B Alignment Philosophy and Tolerances}

The FFTB may be considered to be composed of five straight segments as shown in Figure VI.B.1. These sections are defined by the bend magnets that create the dispersion used in the chromatic correction of the beam line. Each of the sections, except region 1 , is between 30 meters and 50 meters in length. The first segment lies along the SLAC linac axis. We define hinge points to be at the intersections of these straight segments. As we see below, the absolute positioning of the elements of the beam line need not be extremely accurate, but the elements must be held in fixed locations to within a few microns to allow tuning of the beam line optics and to maintain the spot size at the focal point.

\section{Initial Alignment Tolerances}

If the positions of the magnets of the FFTB are assumed to be stable, then simulations of the optical tuning have shown that the spot size at the focal point can be reduced to differ from its design value by only several per cent as long as the magnetic centers (nodal points) of the cuadrupole and sextupole magnets are initially displaced from the local straight line along which they lie by amounts

$$
\delta x_{\mathrm{rms}}<100 \mu \mathrm{m} \quad \text { and } \quad \delta y_{\mathrm{rmus}}<30 \mu \mathrm{m}
$$

Each local line segment must intersect the adjoining segments with a distance of closest approach no greater than $30 \mu \mathrm{m}$ (i.e., the beam line must be locally smooth through the hinge points), but the hinge points need only be within

$$
\Delta x<2 \mathrm{~mm} \text { and } \Delta y<0.2 \mathrm{~mm}
$$

of the design trajectory. Bending magnet power supplies can be adjusted to compensate for errors in geometric angles. 


\section{Stability During Tuning}

Shown in Figure VI.B.2 is the contribution that would be made to the vertical height of the spot at the focal point of the FFTB if any given single quadrupole magnet in the system where moved vertically by 10 microns from its ideal position. As indicated in the previous section, adjustments can be made to correct or compensate for each of these errors, but to do so, the position of the magnets must not change during the tuning procedure. If we demand that the magnet positions be sufficiently stable that no individual motion change the spot size by more than $2 \%$ (either larger or smaller), then we must maintain the position of each magnet to within \pm 2 microns of a location set with respect to the remainder of the magnets in the beam line. Similar calculations show that the roll angles of several elements about their magnetic centers need to be maintained to within 0.2 milliradian. There is one lens (QN2) that may require feedback from measured properties of the beam if it cannot be passively stabilized to $\sim 0.3$ micron.

The stretched-wire alignment hardware, described in Section VI.D below, is designed to monitor changes in the positions of the elements of the beam line with accuracy \pm 2 microns with respect to a line between the hinge points. The system is being designed with overlap between the wires of neighboring straight sections so that the relative motions of the ends of the wires can similarly be determined. Data from this system can be acquired continuously on a minute-to-minute basis.

\section{Long Term Stability}

We anticipate that during periods of use, the optical tuning procedure will yield configurations of magnet positions that produce small spots. We would like to be able to return the magnets to positions that are close to one of these configurations after periods of extended interruption of the operation of the beam line. Our philosophy is that, on these occasions, the calibration of the stretched-wire system may drift substantially, or be compromised by work on the beam line. We are also concerned about the stability of the foundation of the structures that house the beam line. This is particularly true of that part of the line that extends onto the concrete surface of the Research Yard. (See Section VI.F below.) 
We have chosen to provide an "absolute" reference for the FFTB by extending the $3 \mathrm{~km}$ long Fresnel-lens system used to monitor alignment of the SLAC Linac/BSY. The positions of the wires that define the last straight section of the beam line will be monitored with respect to this reference line. Changes in the wire positions need to be determined with accuracies that are substantially smaller than the tolerances on their initial absolute positions. Our goal is to be able to stabilize the wire system to remain within 10 microns of its initial horizontal position and within 5 microns of its initial vertical position.

\section{Magnet Movers}

Each of the quadrupole and sextupole magnets will be placed on remotelycontrollable supports that are capable of translating their lateral (horizontal and vertical) positions over a range of $\pm 1 \mathrm{~mm}$ in steps of 0.3 micron. Trim coils on the backleg flux return of each quadrupole may also be used to move the magnet centers of the focusing elements by small amounts (up to 10 microns). Control of the magnet positions will be done by human operators at the outset of FFTB operation, but as we gain confidence in the alignment system, this operation may be transferred to a microprocessor that will run without human oversight.

\section{Vibration Tolerances}

The alignment system for the FFTB is not intended to be able to correct movements of beam line components that occur faster than can be followed with mechanical magnet movers controlled by a microprocessor-i.e., vibration at frequencies above $\approx 0.01 \mathrm{~Hz}$. To avoid dilution of the final spot size, the critical beam line components will have to be isolated from high-frequency mechanical vibrations with amplitudes larger than a micron.

\section{VI.C Alignment. Techniques}

In this section we present a sequenced approach to achieving the initial placement tolerances for the beam line components and tlieir subsequent maintenance. This approach recognizes the fact that, for radiation shielding considerations during the construction period, it will not be possible to bring a laser alignment path through the iron 
muon shielding block in the BSY and out onto the research yard pad before all construction activitjes are complete.

STEP 1: Extend a conventional network of temporary monuments through the downstream part of the BSY and onto the Research Yard pad. Locations are indicated on the layout (Figure VI.B.1b) by the symbol $\Delta$. Since neither the emerging electron beam direction nor the path of the BSY laser light axis is in a horizontal plane, (they dip about 5 mrad), it is important at the outset to establish the longitudinal coordinates to better than $0.5 \mathrm{~mm}$ over the 300 -meter length of the system. To accomplish this task, a modern Mekometer. ${ }^{1}$ is the instrument of choice

STEP 2: Install Fresnel target stations to form a network that will define the "absolute" reference for the beam line. The Fresnel network is indicated by the symbol $\bigoplus$ in Figure VI.B.1b. One target station will be installed in the vicinity of each "hinge point" to monitor relative motions of the stretched wires with respect the laser reference line. Additional target stations will be installed to complete the network necessary for initial alignment of the beam line. A given target station will provide useful constraints on the alignment of any component if the distance between the two is no greater than ten meters. Each Fresnel station has reference tooling outside its vacuum enclosure, and is equipped with fine adjustment screws that allow final positioning when the laser system is evacuated and activated.

STEP 3: Align by conventional techniques all elements and Fresnel stations within the monument network established in STEP 1.

STEP 4: Install the stretched-wire system. Two wires will be stretched along each segment of the beam line, and at least three read-out sensors will be placed on each quadrupole and sextupole magnet. This will be sufficient to determine changes in the horizontal and vertical positions of each magnet as well as possible changes in the pitch and roll of the magret orientation. 
A laser-tracker ${ }^{2}$ is an instrument that combines a theodolite system to measure angles ( $\delta \theta \approx 2$ arcseconds) with an interferometer-retroreflector system to $\mathrm{F}$ "vide range information. We will use such a device to align the beam line elements well enough that the stretched-wire system can be installed. The laser-tracker is capable of measuring linear distances with resolutions of 5 to $10 \mu \mathrm{m}$, and we estimate that its use at this step will align the elements along each segment of the beam line to $\approx 50$ microns of a straight line in three dimensions.

If the initial network were good enough and stable enough, then the ends of the straight segments would be within specifications, and in the absence of daily ground motions and thermal deformations, the FFTB Fresnel system would not be necessary. However, we believe that the conventional network will not be good enough to meet the initial alignment specificaions of $30 \mu \mathrm{m}$ in the vertical plane. Therefore we must wait until Flesnel targets in the Linac can be used in conjunction with the FFTB targets before we perform this alignment.

STEP 5: The wires serve to detect changes in the alignments of the beam line elements with respect to the wire positions. To fully stabilize the beam line, it is necessary to also monitor movements of the wires themselves. This is to be done in two ways. Each wire pair is to be extended so as to overlap with its neighboring wires. Sensors mounted to fixtures in the overlap regions will monitor the relative displacements of the neighboring wires. This is not, of itself. sufficient to guarantee that the overall layout of the beam line remains fixed, but will provide an internal constraint and monitor. The wire sensors call be read-out continuously minute by-minute.

We will use the Fresnel system to keep track of "absolute" motions of the beam line. Movements of the wires with respect to the Fresnel network will be 
monitored with wire-position sensors mounted on small invar platforms similar to the tooling frames used on the beam line magnets. A Fresnel target station will be located near to each wire-position platform. It is assumed that deep within the BSY channel conditions will be stable enough that the positions of beam line components with respect to nearby

Fresnel targets will not change by more than 10 microns on a daily basis and not more than 20 microns on a monthly basis. Measurements indicate that distortions of the Linac housing are within these bounds. On the other hand, we know that the concrete pads that make up the surface of the Research Yard move with respect their surroundings by considerably greater amounts. (See Section VI.F below.) A schematic plan of the system that will be installed to monitor the alignment of the last straight section of the bearn line is shown in Figure VI.C.1. "Bridges" will be used to track the motion of each wire-position platform with respect to an adjacent Fresnel target station. The Bridge hardware is described below. 'The Fresnel system can be read-out only slowly since only one lens at a time can be inserted into the laser beam. A complete survey requires an hour or more to acquire, although partial surveys can be obtained more rapidly.

STEP 6: Once all parts of the FFTB including the shielding are in place, and the plugs in the iron wall removed, Fresnel targets in the linac can be used to define the reference for the FFTB. The Fresnel stations are then to be used as a network for the "absolute" alignment c " bean line. We will use the laser-tracker to measure and correct the locations and orientations of all eiements along the beam line. The locations of the wires can also be recorded at this point for future reference. This process is shown in Figure VI.C.2. The errors that we expect to incur in this process are summarized in Table VI.C.1. The elenents of the beam line should be easily within the required positioning accuracies of $30 \mu \mathrm{m}$ in the vertical plane and $100 \mu \mathrm{m}$ in the horizontal plane. 
STEP 7: The stretched-wire system is activated by nulling all wire sensor readings and beginning to track magnet and wire positions. The accuracy with which online moaitoring and reconfiguration of the beam line can be done is summarized in Tables VI.C.2-4.

\section{VI.D Alignment Hardware}

\section{The Magnet Reference Tooling Frame}

The effective magn-ic center of a focussing element, or the electrical center of a beam position monitor: - not a physical location that can be touched with alignment tools. Some form of practical reference tooling has to be provided. Traditionally this has been in the form of tooling balls mounted in some convenient place on the object with the traditional confusion of just where these balls are relative to where the beam thinks the center of the object is. Moreover, thermal expansion of the object changes the location of these tooling balls. The frame, shown sketched in Figure VI.D.1 overcomes some of ihese problems. The legs are brought to bear on the split planes of the magnet, which being symmetry planes, should not change their location with expansion. The legs are made of invar or other material of very low expansion coefficient.

\section{The Stretched Wire System}

Stretched wires have been used since Egyptian times to align large structure. ${ }^{3}$ Development of high precision wires for this application has been undertaken by the DESY laboratory. 4

A spring steel wire of $0.5 \mathrm{~mm}$ diameter has been stretched over a distance of $45 \mathrm{me}$ ters using 42 Kilogram tension. The observed sagitta was an acceptable $6 \mathrm{~mm}$. Interestingly it was found that the wire sag was better fit with a parabolic function than with the theoretically predicted catenary. Most importantly, the wire's natural oscillations (frequency $6 \mathrm{~Hz}$ ) are damped with a time constant of 16 seconds. An amplitude-vs-time display lasting 2.5 minutes is shown in Figure VI.D.2. The residual motion due to nataral microseismic motion of the supports and/or air drafts is shown in Figure VI.D.3. Long termi measurements of the vertical and horizontal positions of the wires showed 
very slow drifts of less than 20 microns over days. These drifts are thought to be more indicative of the tunnel stability and its environment than of the wires themselves. All measurements were carried out using shadows of the wires cast on CCD arrays.

A sensor for the wire, based on BPM-style electrical pickups, is being designed. These devices will have physical apertures of at least $3 \mathrm{~mm}$ to allow reasonable clearance about the $\pm 1 \mathrm{~mm}$ range of the magnet movers. Either short current pulses or continuous $r f$ waves will be sent along the wire to induce signals in the monitor electrodes. Electrical read-out and digitization with 12 -bit $A D C$ s will provide a least-count no larger than 0.5 micron, a full range of $\pm 1 \mathrm{~nm}$, and a range over which the system is linear to within one least-count of greater than \pm 100 micron. The wire, detection elements, and electronics in this alignment system should be free of internal drifts of magnitudes comparable to the sesolution of the system over the period of a day.

\section{The Fresnel Target Laser System}

An exploded view of an existing laser station showing its saddle, vacuum housing, leveling plate and actuator assembly is shown in Figure VI.D.4. The laser itself and its mount will be mounted in the research yard outside the new housing so that, in contrast to its previous location, it could be manipulated during beam operation. We intend to maintain the existing geometry in which the light enters the housing at right angles to the linac axis and note that since this location is likely to move about during operation, we will employ readings from Fresnel lenses chosen from each end of the linac to define the reference line for the FFTB.

On April 25, 1990, it was possible to reactivate the original BSY laser system and to take data with it, as well as with the main Linac Laser Alignment system. The positions of images were marked on a ground glass screen, rather than with the usual trolley, because they were much displaced with respect to preliminary observations taken in February 1989. The analyzed data (corrected for station pedigree only in the linac) and referring to a straight line with the laser itself on one end and the center cf the glass port in the detector room as the other end, is shown in Figure VI.D.5.

Figure VI.D.6 displays a magnified view of the switchyard data. Ignoring the points from the monument (which was never used for BSY alignment) and station 11 (which 
was moved during SLC construction) the horizontal readings average to -1 mils and have an rms spread of 10 mils. The vertical readings cluster around -57 mils and have a spread of 17 mils. Our recollection from February 1989 is that the vertical data was tighter then but it should be mentioned that the new data was not only post earthquake but also after many tons of iron shielding were rearranged in the switchyard. These results are very encouraging with respect to the stability of the old BSY tunnel floor.

\section{The Bridge}

The purpose of the "Bridge" is to reference the ends of the wires to the absolute coordinates of the laser stations. A cross section depicting this function is shown in Figure VI.D.7 Since the coordinates of these locations are vastly different in both the horizontal and vertical planes, it is important to maintain the local coordinate frame plumb. Other parts of the bridge include the extensometer, mercury height transfer liquid levels and tiltmeters.

For the past year we have been attempting to develop a precision mercury level to measure height differences in the micron range. The principle requirements on the device are that it provide reproducible results over long periods without drift and be reasonably radiation hard. When it was pointed out to us that oxygen, a constituent of air, attacks the mercury surface, thereby altering surface tension and hence changing the meniscus determined height of the liquid, we returned to an all metal (stainless steel) system that can be evacuated and backfilled with dry nitrogen. Various height sensors were examined. The one used in the current version is a capacitance sensor ${ }^{5}$ which is housed outside the cell volume and which measures the liquid level by sensing through a thin plastic window (polycarbonate). The assembly is shown in Figure VI.D.8. For these tests the usable range had been set to $\pm 0.5 \mathrm{~mm}$ per cell. The height of the mercury pool is $12 \mathrm{~mm}$. No attempt has yet been made to compensate for lieight changes due to cell temperature variations. These are expected to be $0.5 \mathrm{microns} / \mathrm{deg}$.C. The free period of the instrument is expected to be about 120 seconds.

A one week portion of a two month trial of two cells (connected 2 meters apart) resting un the concrete floor of the Central Laboratory Annex basement are shown in Figure VI.D.9. Data was logged every ten minutes. A tilt of 1 microradian represents 
one cell rising, the other falling by 1 micron. The data shown in this figure was taken after covering the cells with thermal insulation and shows that after several days the cell temperature stabilized at the ground temperature. The other temperature trace represents the air temperature in the room near its east wall. A check made with the aid of a third sensor measuring a fixed distance showed that the signal processing electronics was insensitive to ambient changes, varying no more than one digitizer count for the ambient changes observed. One digitizer count is 179 microvolts and represents an 18 nanometer liquid height difference.

Our current interpretation of the data is as follows. Since ambient temperature changes of the instrument cannot explain the apparent tilt of the floor, we will assume that it is real. The measured period of the oscillation is much closer to 24 hrs than 24.8 hirs (the period of the moon) and we therefore ascribe the motion to thermal effects outside the experiment rather than to the earth tides. The ambient air temperature in the room follows the temperature outside of the building. We therefore speculate that the sun heats the East wall of the building preferentially and thereby tilts the basement floor. (The south wall of the basement is underground.) One notices that the swings of temperature and motion are smaller on cooler days which have overcast skies. Recent tests conducted in the sector 10 test bench show similar daily fluctuation in tilt. (See Figure VI.D.10.)

We are encouraged and work of this kind continues on smaller more practical cells, differential eddy current sensors, ${ }^{6}$ other liquids for the level and temperature compensated mountings.

\section{Inclinometers and Rotation Tables}

Using a high resolution tilt sensor mounted on a rotation table, it is possible to compute the absolute, gravity relative, tilt of a plat form or tooling frame upon which wire position monitors and/or proximity sensors are nounted. The Final Focus Test Beam alignment system will use wire position nonitors and proximity gauges to track motions of the magnetic centers of focusing magnets at the nicron level with respect to an absolute reference frame. The "tracking" measurements are performed at some distance, tens of centimeters, from the points being traclied. Assuning that the distance between 
the points being tracked and measurement devices is constant, or can be computed, one must nevertheless know the angular orientation between the measurement devices and the points being tracked. The inclinometer system provides this information. The rotation tables allow to balance systematic errors (bias, zero offset).

The planned for resolution of the sensors is 1 arc seconds ( 4.85 microradians).

\section{Controls}

The style and architecture of the controls system is constrained by the fact that FFTB operation is part of a much larger accelerator system, namely the SLC whose controls are CAMAC based and VAX driven. The very complexity of SLC also imposes certain uniformity standards on hardware and particularly software issues. In order to be operationally compatible with SLC the designs of magnet controllers, beam position and profile monitors, magnet mover controls and the like, are carried over directly to the new system; their overhead has already been accounted for. Less clear have been the issues connected with devices that have no clear precedence such as the readout of the laser alignment system images, wire position monitors, proximity sensors tiltmeters and the like. The first cut on their integration is shown in Figure VI.D.11. Those systems requiring local processing are provided with micros that then communicate with the VAX employing standard protocol.

\section{VI.E Magnet Movers}

All magnetic lenses will be equipped with "magnet movers" to mechanically translate them back into line when their position has drifted by unacceptable amounts. It is important that mechanical translations can be carried out on-line, that is wlile the beam is in operation. While it is relatively easy to move objects as heavy as magnets horizontally on precision slides, vertical motion is harder to achieve.

An elegant system of "cam actuators" has been developed to position the final lenses of the SLC final focus. ${ }^{7}$ A sketch of the original device, providing two translations and three rotations of a rigid body is shown in Figure VI.E.1. A new design, suitable for most FFTB components but having only three degrees of freedom $x, y, \theta_{z}$ has been completed. It is shown in Figure VI.E.2. Interesting features include a "harmonic drive" 
for the gear reducer box and precision $(<1 \mu)$ LVDT based coordinate readout. Coarse adjustment of its base is provided for at the $\pm 5 \mathrm{~mm}$ level. We assume that the final triplet assembly and the four quad-sextupole-quad packages will be mounted on the five degree of freedom type mover. The group at KEK will design and fabricate the mounts for the magnets that make up the final lens doublet.

\section{VI.F Some Recent Measurements on the Research Yard Site}

In contrast to the heretofore mentioned stability of the $\mathrm{C}$ line under the mountain of shielding that covers the BSY, the research yard is in the open and subject to the effects of the weather.

\section{Pier Thermal Motion}

An experiment to determine the thermal stability of the research yard base, carried out in the summer of 1989 , concluded that portions of the concrete pad moved with respect to the base of End Station $A$ by as much as $0.6 \mathrm{~mm}$ on a daily basis as a result of pad heating by the sun. It was therefore recommended that concrete piers be sunk into the bedrock which were to be suitably decoupled from the surface of the concrete pad and therefore immune to surface temperature effects.

Three piers, each $3 \mathrm{ft}$ in diameter and $11 \mathrm{ft}$ deep were subsequently poured in November 1989. They were designed to simulate the supports of the final focus test beam final quads. Measurements on their lateral position (ie., w.r.t. End Station A) were begun March 24, following a four month waiting period-long enough after pouring to ensure that the concrete had fully cured.

In contrast to the earlier experiment in which a Kern Mekometer was used at the limits of its stability, changes in length of the 33 meter distance were now measured using a Hewlett-Packard interferometer. As before, the flight path was evacuated by means a 4 -inch diameter tube to make the readings insensitive to changes in atmospheric temperature and pressure. The piers in the yard were shielded from the sun by a large tin roof, its sides were loosely covered with a tarpaulin. The wind could blow through the structure freely. 
Figure VI.F.1 shows results of the first data set. The data is smooth (in comparison to that taken with the Mekometer) but shows almost as much motion as the previous experiment. Although the figure shows a complete daily cycle it is stitched together from three separate days because the interferometer would on occasion "loose count" when something caused the laser beam to become misaligned. The total amplitude of motion is 300 microns (as opposed to 600 in summer 1989) however the temperature swings in March were not as extreme as last July.

A series of experiments were conducted next to ascertain whether the results were real or instrumental. Even though both mekometer and interferometer results are very similar, one might suspect that the interferometer head is sensitive to temperature changes. For this reason the apparatus was moved further away from the door sill of and into ESA by extending the vacuum pipe another $15 \mathrm{ft}$. The fixed retroreflector on the pier (insulated against temperature changes) was replaced by one mounted on a servo-controlled frame which could track minute changes in laser direction so that the instrument would not loose count for days at a time. The tilt of the door sill was monitored with a resolution of a milligon. The results are displayed in Figures VI.F.2. They are not easy to interpret. As before the motion lags the temperature of the concrete by about three hours when the temperature rises. However, just prior to the rise, the motion is reversed as if the floor of ESA suffers a hysteretic excitation. The data clearly shows that when the sky is overcast and lesser concrete heating occurs, the motion can be as low as 50 microns.

The notion that the evacuated pipe had a leak (in both experiments) and that temperature changes of the residual gas were responsible for the observations was checked by shielding the pipe from the sun. The results were unaffected by the shielding. Changes in temperature of the port windows also cannot explain the observation. At that moment we did not know what moved, the pier or the end-station but it became clear that our simple notion of decoupling the two by pouring a pier into the miocene bedrock was not yet a satisfactory answer.

The next experiment in this series was to measure variations in the distance between the piers and End Station B. The walls of the building face in the opposite direction to 
the sun from those of End Station A. The results are shown in Figure VI.F.3. As the temperature climbs with the rising sun the distance between ESB and the pier becomes smaller and one notes by a similar amount that the distance to ESA gets larger. This fact leads one to the interpretation depicted in Figure VI.F.4. The changing azimuth of the sun causes differential heating of the surface and some small fraction of the resulting motion is transmitted to distort the miocene below.

Recent Mcasurements of Vibration Levels on Central Beam Piers

In order to ascertain whether or not an active vibration isolation system ${ }^{8}$ would be required for use in stabilizing the last lenses of the FFTB, a series of vibration level measurements were carried out.

Vertical and Horizontal velocity transducers were placed on the outer piers. A typical vertical velocity spectrum is shown in Figure VI.F.5. The most prominent peak occurs at $29.875 \mathrm{~Hz}$. This frequency is known to be related to LCW cooling water pumps. The minor peaks at 11.6, 12.25 and 13 are believed to be associated with machinery in the nearby cryogenics facility. With a sensitivity of $1 \mathrm{~cm} / \mathrm{sec} \Longleftrightarrow 1$ volt, the 55 microvolt rms signal translates to a rms coherent amplitude of 0.003 microns. The lower frequency peaks contribute comparable amounts. The rest of the spectrum is barely above the noise of the analyser. These measurements must be repeated when the new rapid cycling injector synchrotron for SSRL operations of the SPEAR storage ring becomes operational.

Figure IV.F.6 depicts a real time trace of a time integrated signal proportional to real time amplitude. With an integrator gain and time constant yielding 0.8 microns/volt calibration we see peak to peak East West amplitudes up to 0.5 volts, i.e., 0.4 microns. The period of these oscillations is very long (about 3 seconds) and therefore not considered particularly dangerous. (For discussion see below) Note: The roof and wall of the shed covering the apparatus was subjected to a fair amount of wind while this measurement was made. Figure VI.F.7 depicts the Probability Density Function of this real time horizontal motion to be equivalent to about 0.1 microns. By 17:55 hours the wind had died down and the EW motion had fallen a factor of two. The veritical motion (See Figure VI.F.8) remained equivalent to about 0.05 microns rms. 
A measurement of the shear wave velocity in the bedrock is afforded by dropping a lead brick and measuring the time of arrival at vertical sensors mounted on the outer piers as shown in Figure VI.F.9. The distance between the piers is 5.5 meters and the arrival time difference which can be read from Figure VI.F.10 is 14 milliseconds yielding a not unreasonable shear wave velocity, for this material at this depth, of about 400 meters/second. Since all disturbances with wavelengths greater than say 75 interpier distances will be $92 \%$ coherent $(\sin 2 \pi / 75 \approx .08)$ flequencies below $1 \mathrm{~Hz}$ will not be dangerous to this extent. However larger distances require the absence of disturbances above still lower frequencies. Generally speaking, the larger amplitudes (up to 1 micron) due to ocean wave activity on the Pacific rim occur only during heavy winter storms at sea and have periods in the many seconds. 


\section{References}

1. For example, the ME5000 made by Kern, Switzerland.

2. For example, Chesapeake Laser Systems, Inc., 4473 Forbes Blvd., Lanham, MD 20706.

3. Some more recent applications may be found in "Alignment and Vibration Issues in TeV Linear Collider Design," G.E. Fischer, Particle Accelerators 1990, Vol. 31, p. $47-55$.

4. "Wire Measurements for the Transversal Control of tice FFTB-Magnets," W. Schwarz, Paper D4, Second International Workshop on Accelerator Alignment DESY, Hamburg, Germany, September 10-12, 1990.

5. Model HPT-375G-A-12-100-B-D, Capacitec, P.O. Box 819, 87 Fitchburg Rd., Ayer, Mass 01432, (508) 772-603, with serjes 4000 signal processing.

6. Kaman Instrumentation Corporation, 1500 Garden of the Gods Rd., P.O. Box 7463, Colorado Springs, CO 80933, Model KD-5100 Series, used to steer telescope mirrors in the microradian range.

7. Final Focus Memo "A Positioning Mechanism for the Final Quadrupole Triplet," G. Bowden, G. Putallaz, March 10, 1985.

8. See for example, N.Ishihara et al., Particle Accelerators 1990, Vol. 31, p. 57-62. 
Table VI.C.1 Accuracy of the Laser-Tracker Alignment of the FFTB

\begin{tabular}{lc}
\hline \multicolumn{1}{c}{ Item } & Error (microns) \\
\hline Change in position of magnetic center: & 5 \\
Node-finding wire to magnetic center: & 2 \\
Fiducial flange to node-finding wire: & 3 \\
Tooling frame to fiducial flange: & 7 \\
Tooling frame to actuator: & 10 \\
Tilt of actuator: & 3 \\
Actuator tooling surface to mechanical center of grating: & 7 \\
Mechanical vs optical center of diffraction grating: & 5 \\
Center of diffraction image: & 3 \\
Actuator nonrepeatability: & 5 \\
Z position of lens stations: & 2 \\
Movement of laser over one cycle: & 7 \\
& \\
& Quadrature Sum:
\end{tabular}


Table VI.C.2 Accuracy of the Stretched-Wire System (Short Term)

\begin{tabular}{ll}
\multicolumn{1}{c}{ Item } & Error (microns) \\
\hline Change in position of magnetic center: & 1 \\
Nonsystematic rtotion or distortion of wires: & 1 \\
Wire position sensor resolution: & 2 \\
Thermal gradients in tooling frame: & 1 \\
Response time of the control system: & 1 \\
(Motion which is too fast to be corrected) & \\
\hline & Quadrature sum: \\
\hline
\end{tabular}

Table VI.C.3 Accuracy of Daily Alignment of the FFTB

\begin{tabular}{|c|c|}
\hline Item & Error (microns) \\
\hline Tilt of beam pipe platform $(20 \mathrm{~min})$ : & $<1$ \\
\hline Drift of bridge sensors $(20 \mathrm{~min})$ : & $<1$ \\
\hline Tilt of actuator $(20 \mathrm{~min})$ : & $<1$ \\
\hline Center of diffraction image: & 3 \\
\hline Actuator nonrepeatability: & 5 \\
\hline Movement of laser over one cycle: & 7 \\
\hline Quadrature sum: & 9.1 \\
\hline
\end{tabular}


Table VI.C.4 Accuracy of Weekly Reconfiguration of the FFTB

Itenı Error (microns)

Change in position of magnetic center: 5

Change in position of tooling frame (weekly): 3

$\begin{array}{ll}\text { Wire position monitor drift (weekly): } & 10\end{array}$

Nonsystematic motion / distortion of wires (weelly): $\quad 3$

Tilt of beam pipe platform (weekly): 3

Drift of bridge sensors (weekly): 5

Tilt of actuator (weekly):

Center of diffraction image: 3

Actuator nonrepeatability: 5

Movement of the laser over one cycle: $T$

Quadrature sum: $\quad 16.4$ 


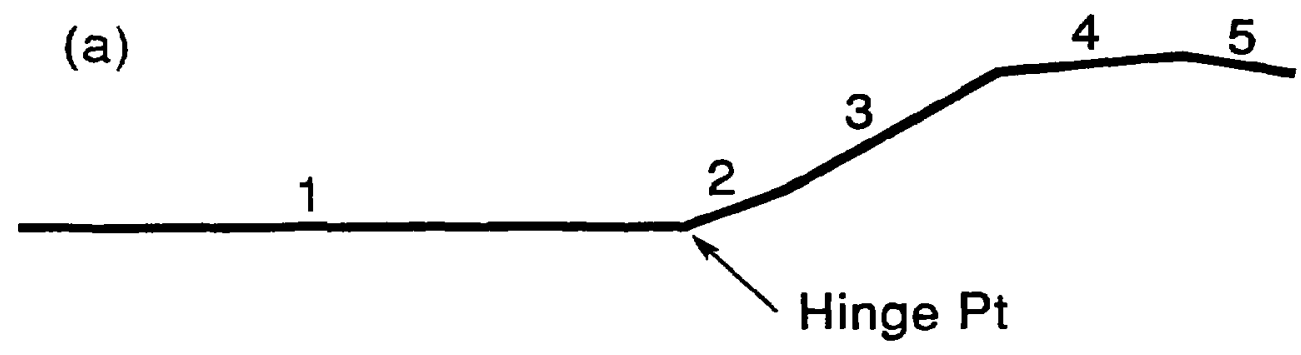

(b)

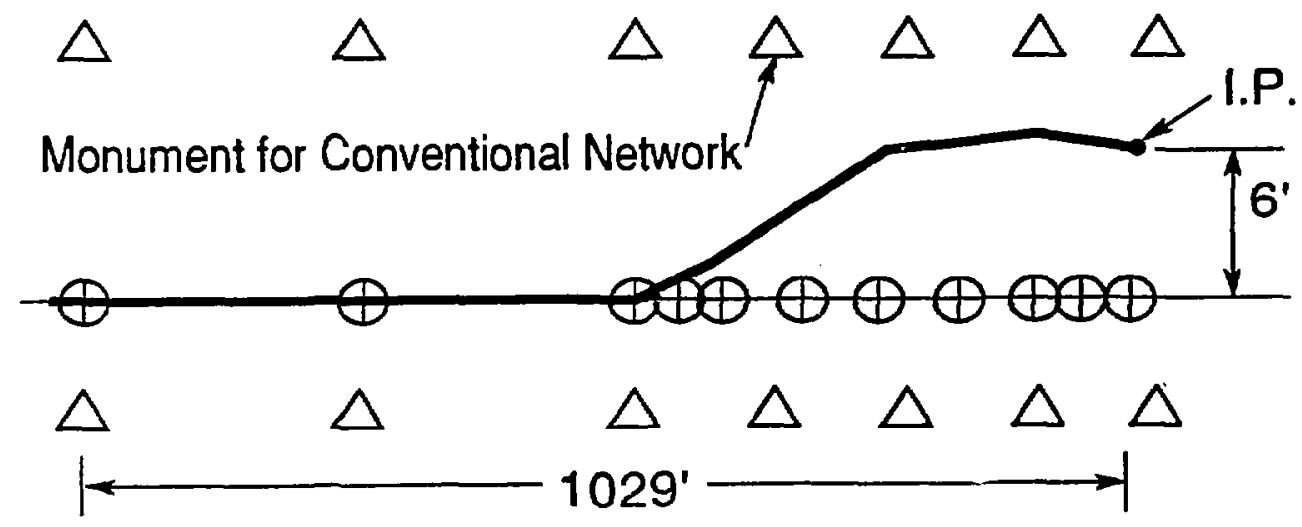

Figure VI.B.1. (a) Equivalent FFTB Geometry in terms of straight line segments. (b) Layout of the conventional alignment and Fresnel target networlis for the FFTB. 


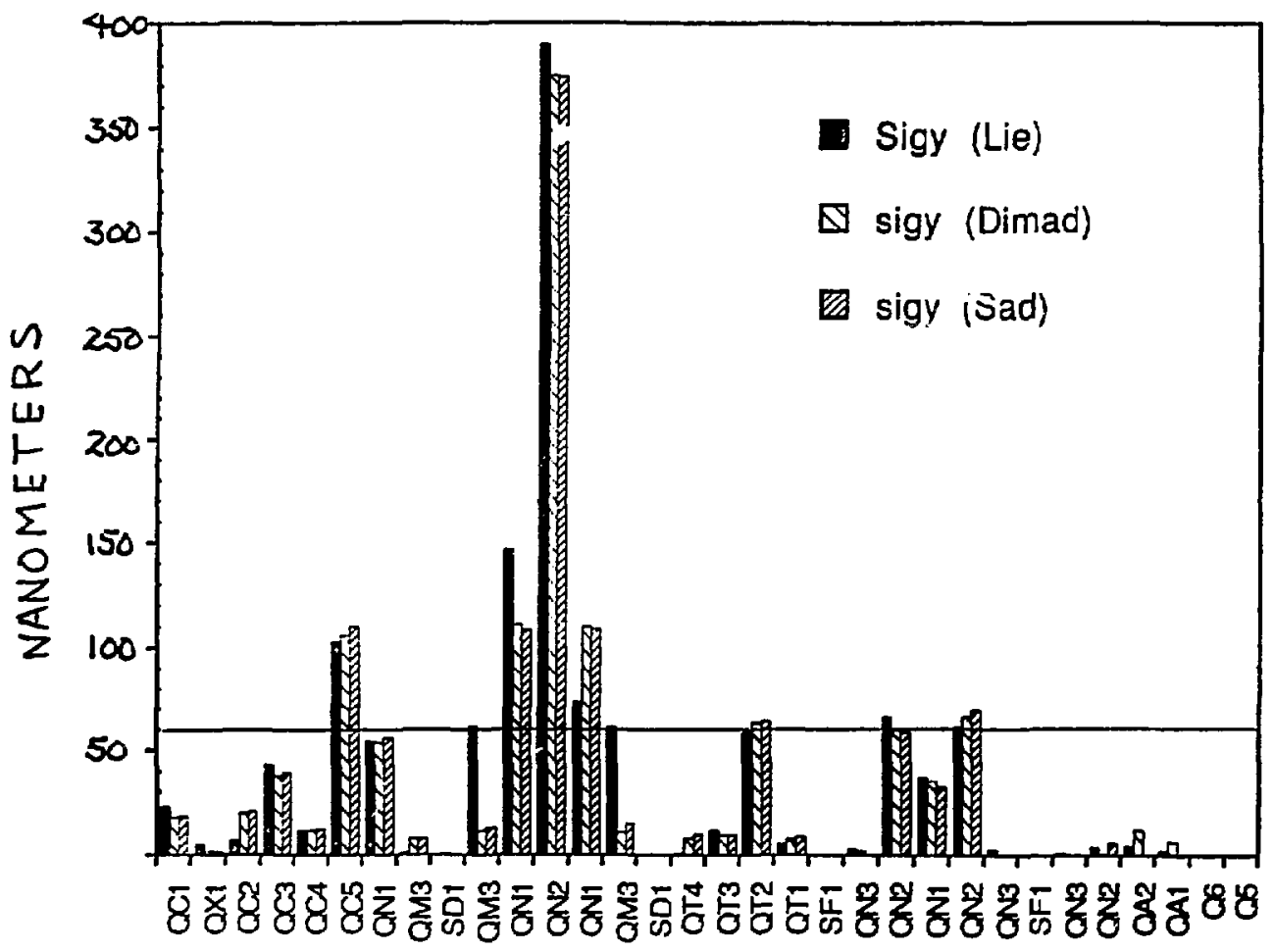

Figure VI.B.2. Contribution to the vertical height of the beam spot at the focal point generated by 10 microns of vertical motion of various magnets. 


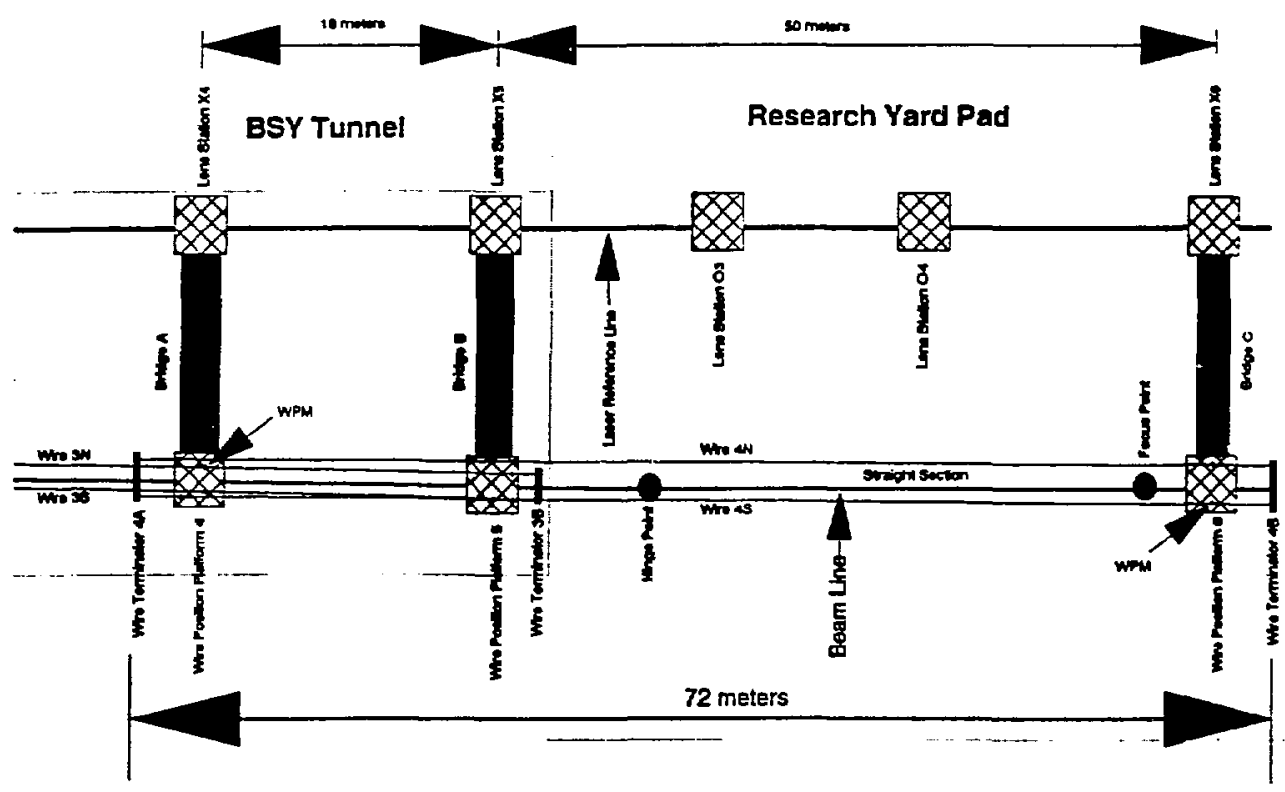

Fjgure VI.C.1. Layout of the alignment apparatus in the last straiglit section of the FFTB beam line. 


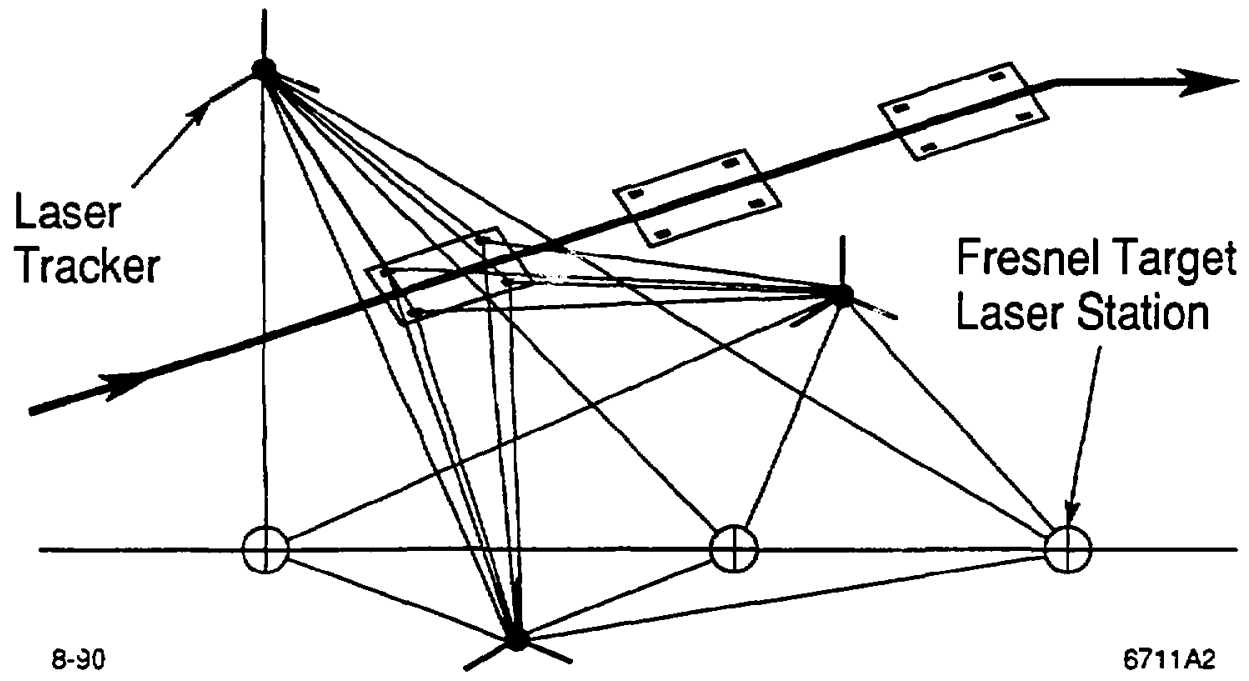

Figure VI.C.2. Use of a Laser Tracker and Fresnel network for initial alignment of the beam line elements. 


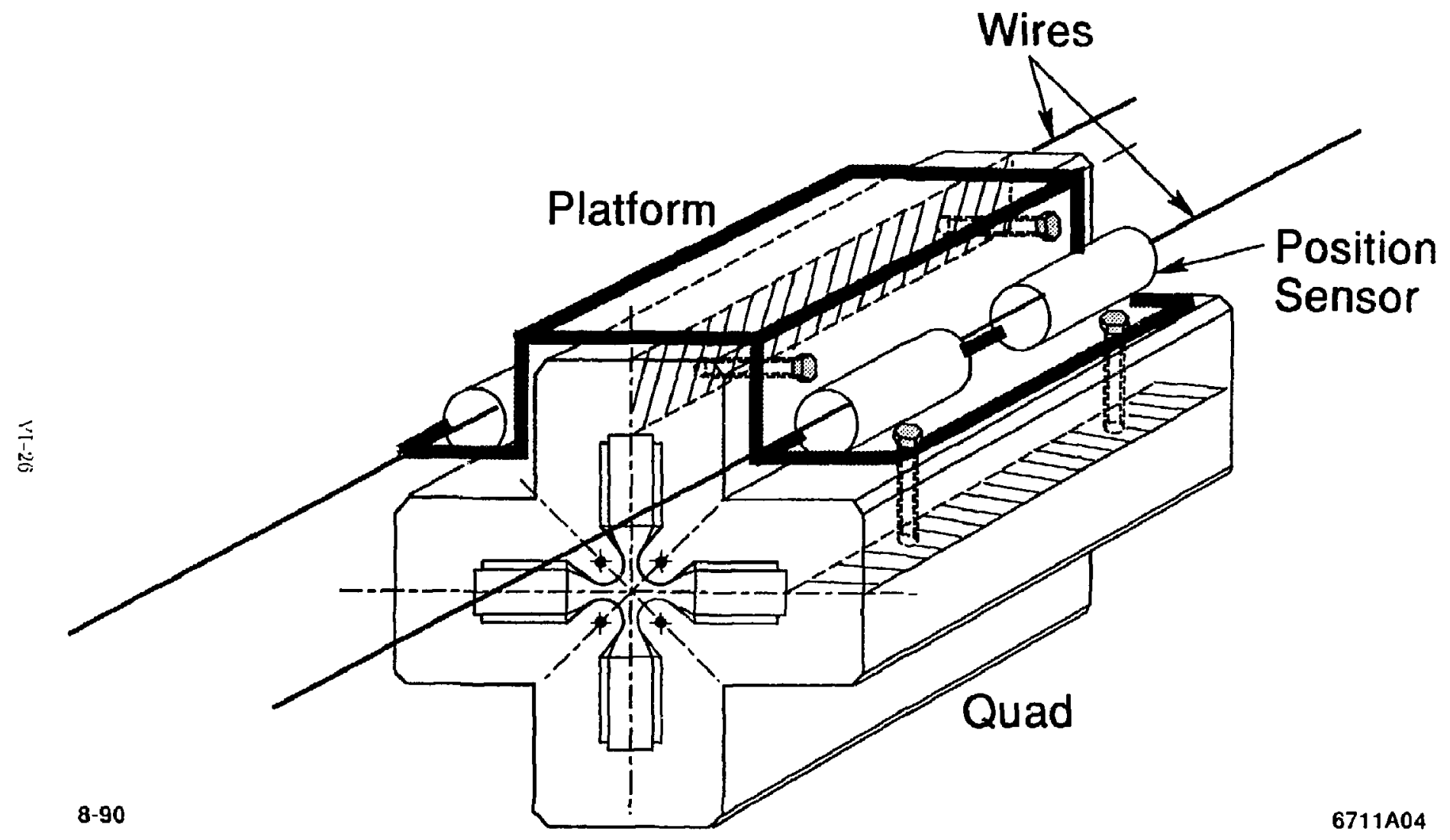

Figure VI.D.1. Artists Conception of the Magnet Reference Tooling Frame. 


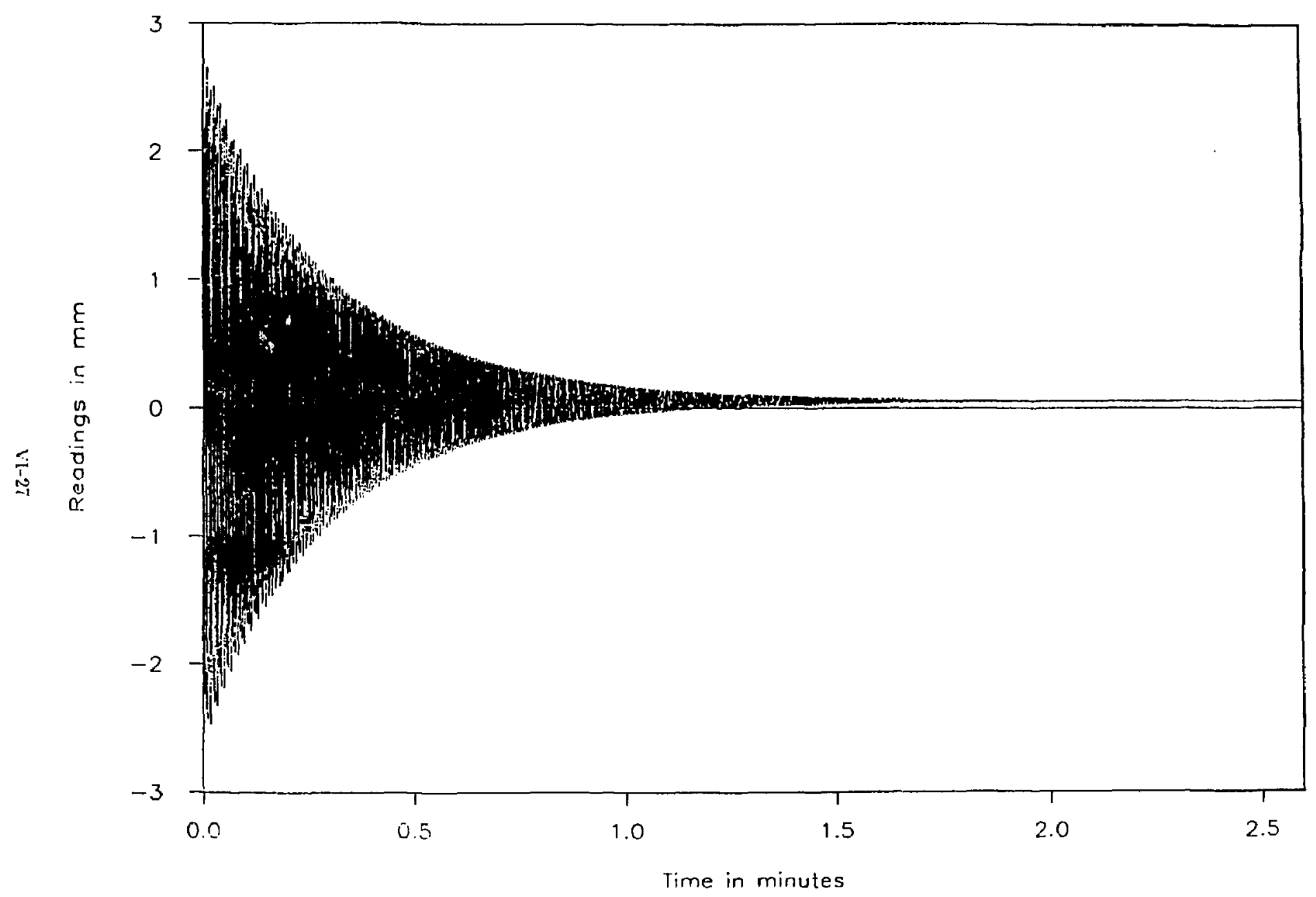

Figure VI.D.2. Decay with time of the transverse amplitude of oscillation of a plucked spring steel wire. 


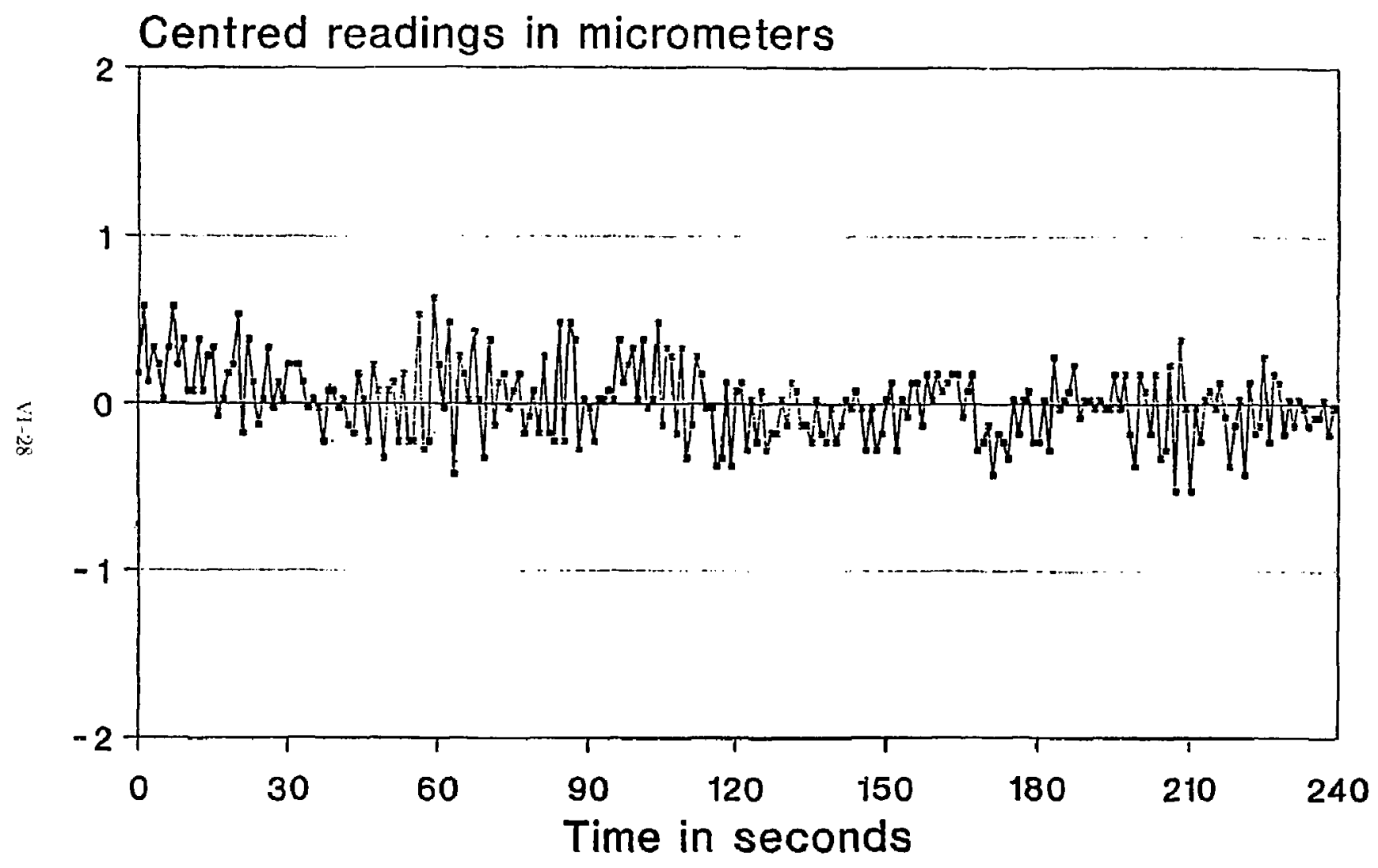

Figure VI.D.3. Residual motion of the test wire in microns. 


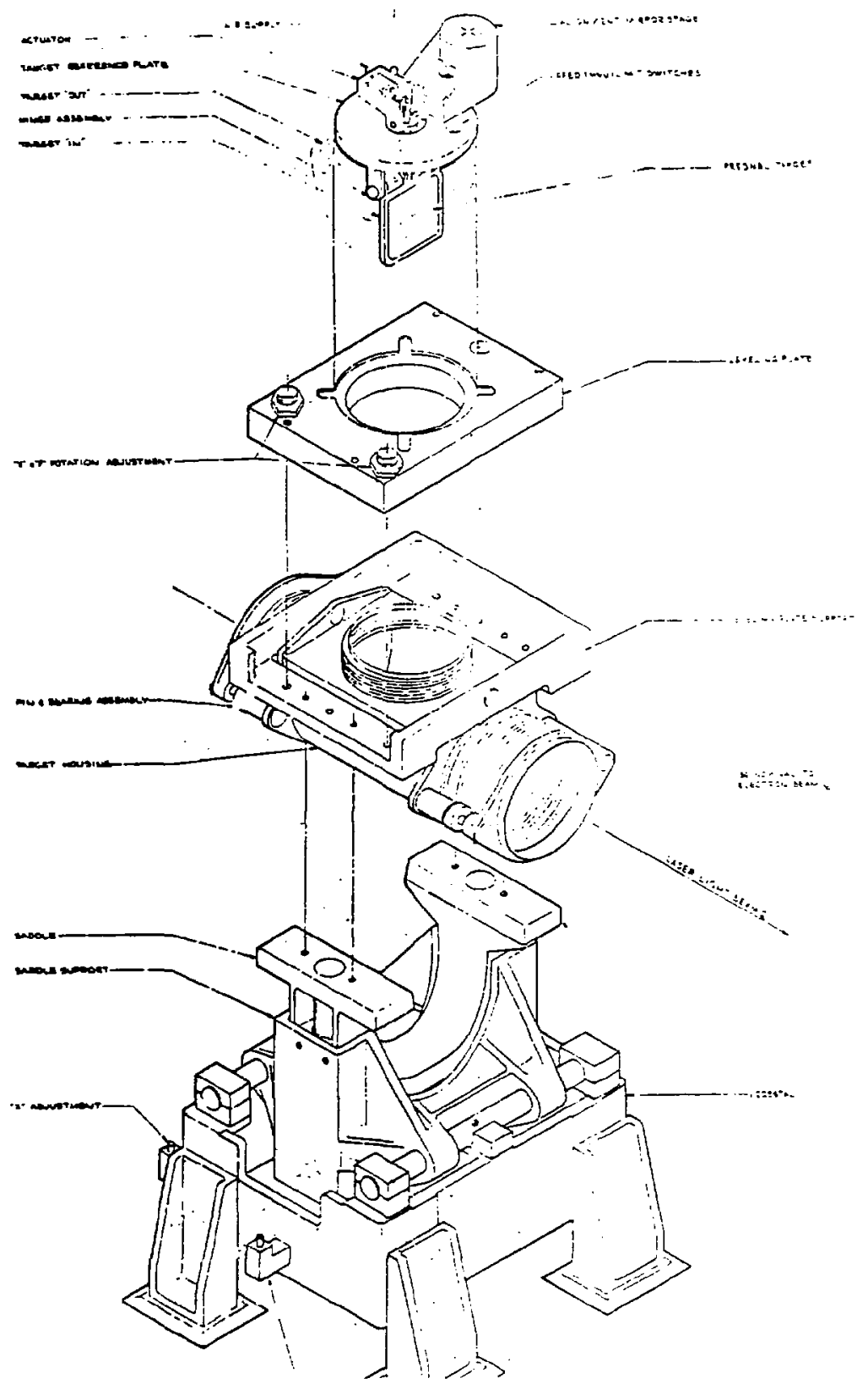

Figure VID.4. Explorled riew of a BSY laser station. 


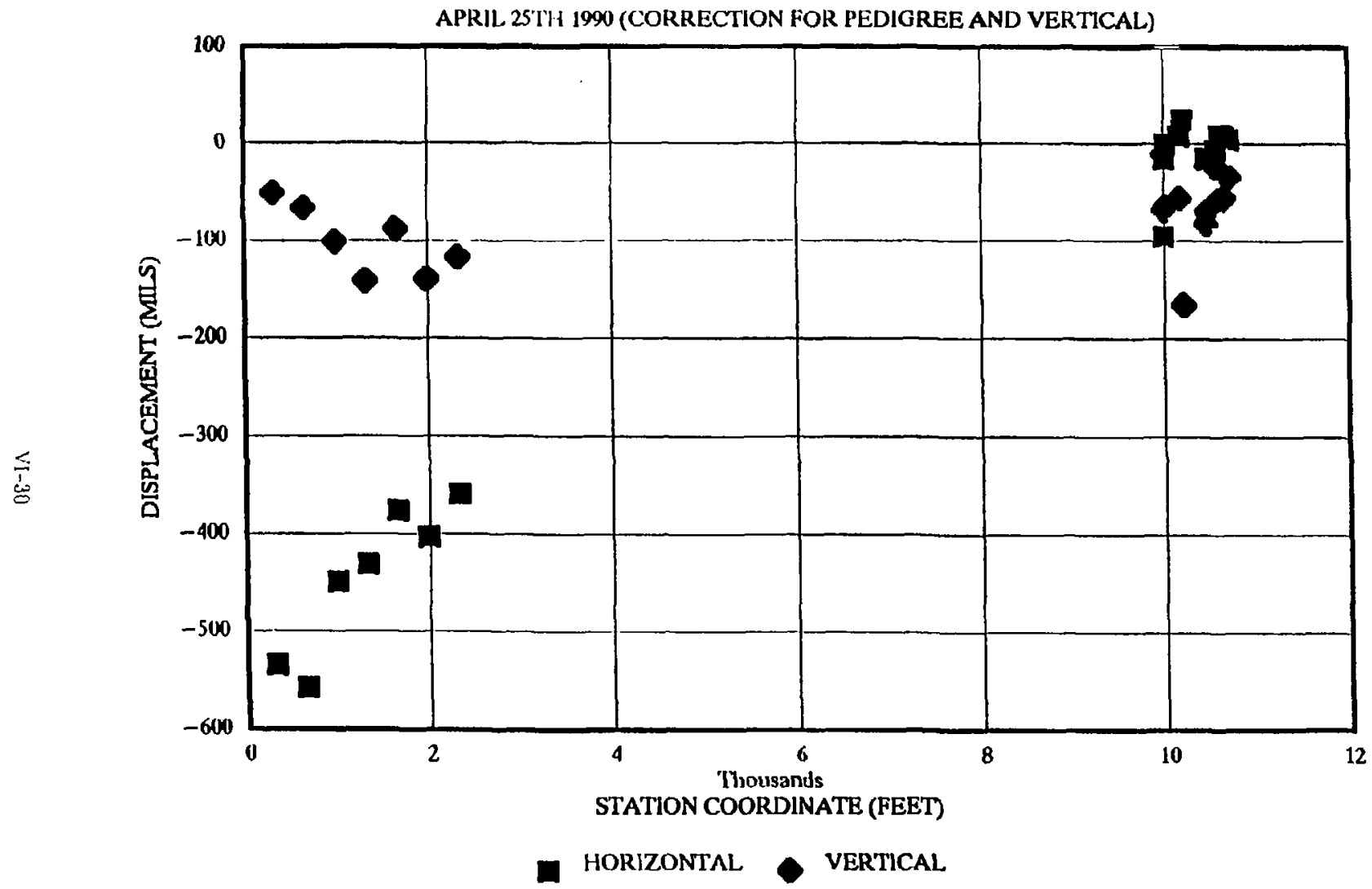

Figure VI.D.5. Relative alignment of Laser Stations in the BSY (last moved in 1966). 


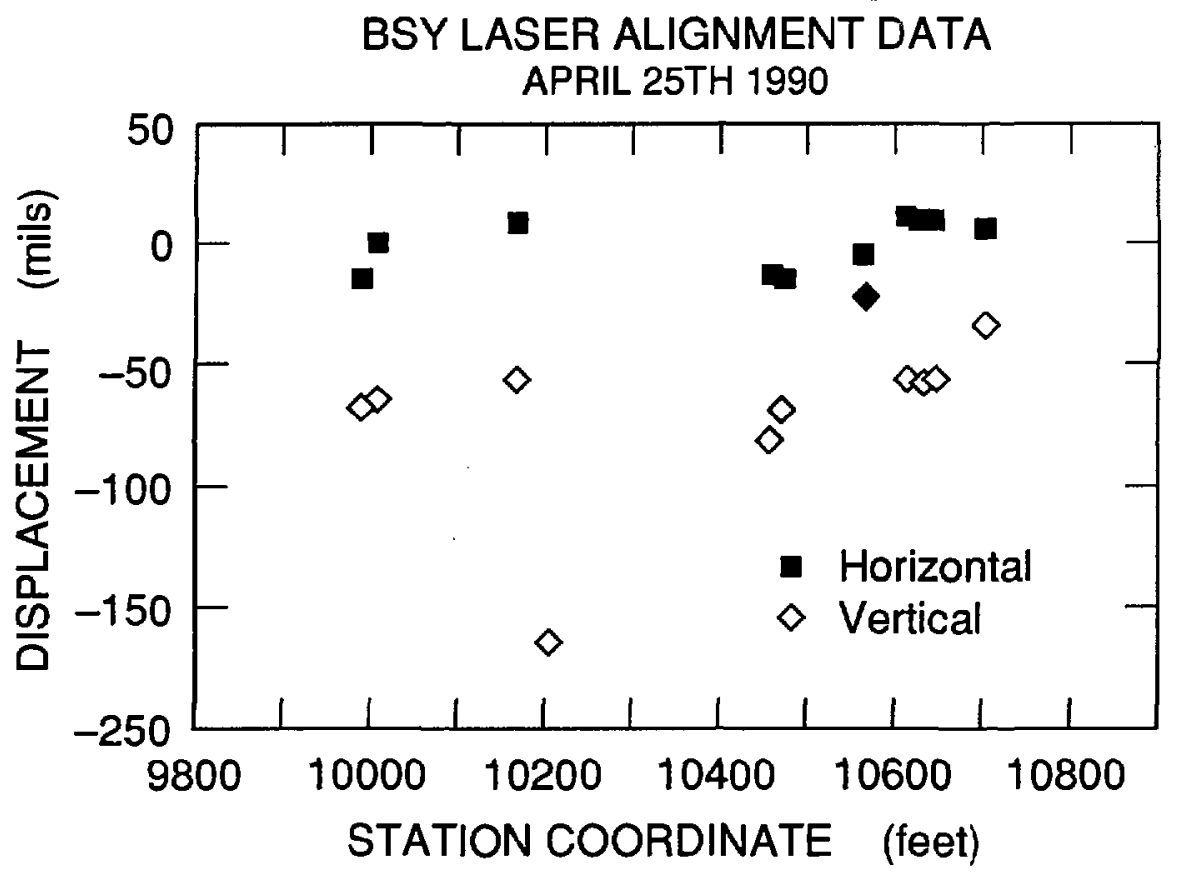

Figure VI.D.6. Magnified view of laser lens positions in the beam switchyard. 


\section{BEAM SWITCHYARD CROSSECTION}

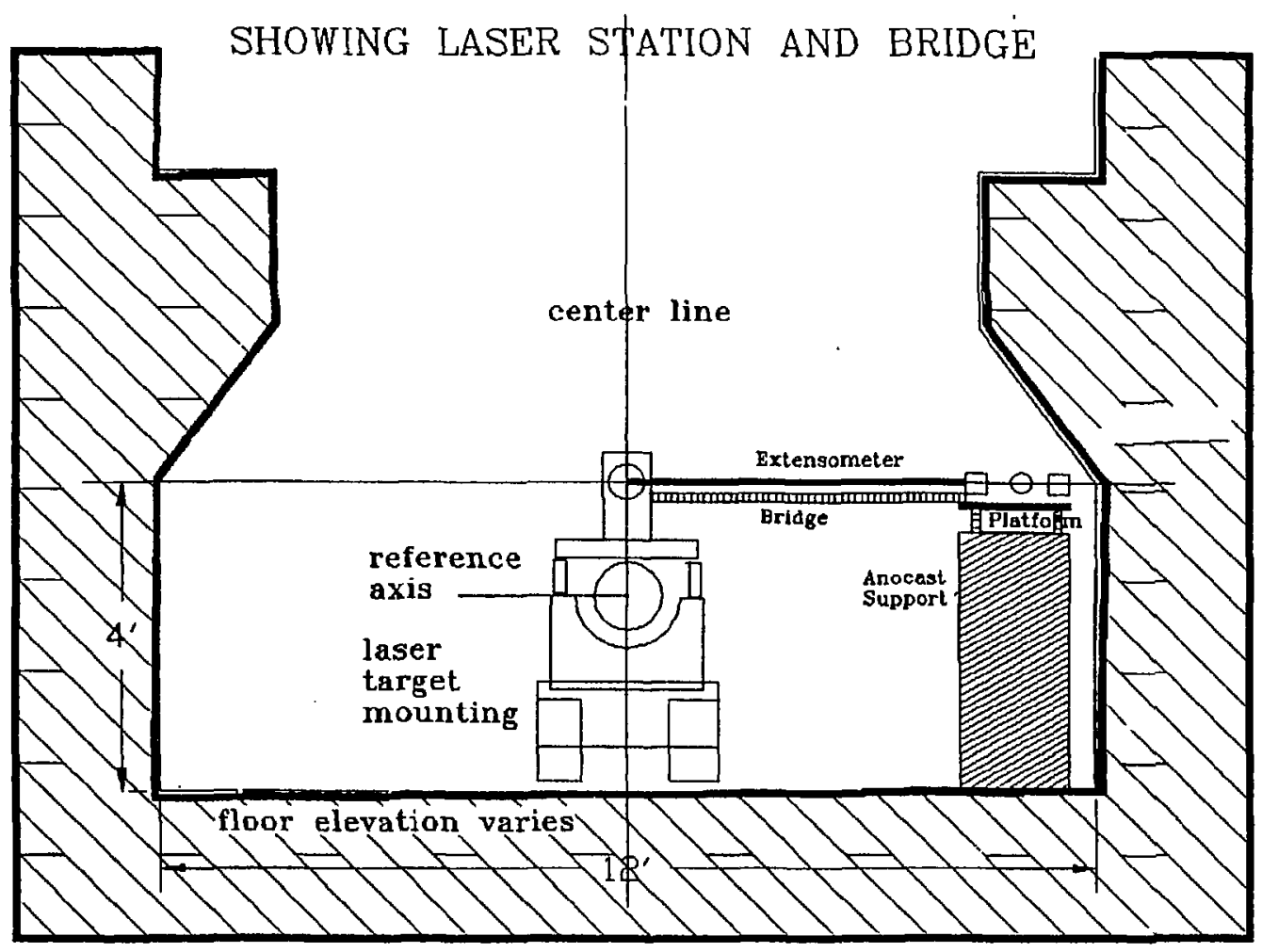

Figure VI.D.7. The Coordinate Transfer Bridge (View: along the beam direction). 


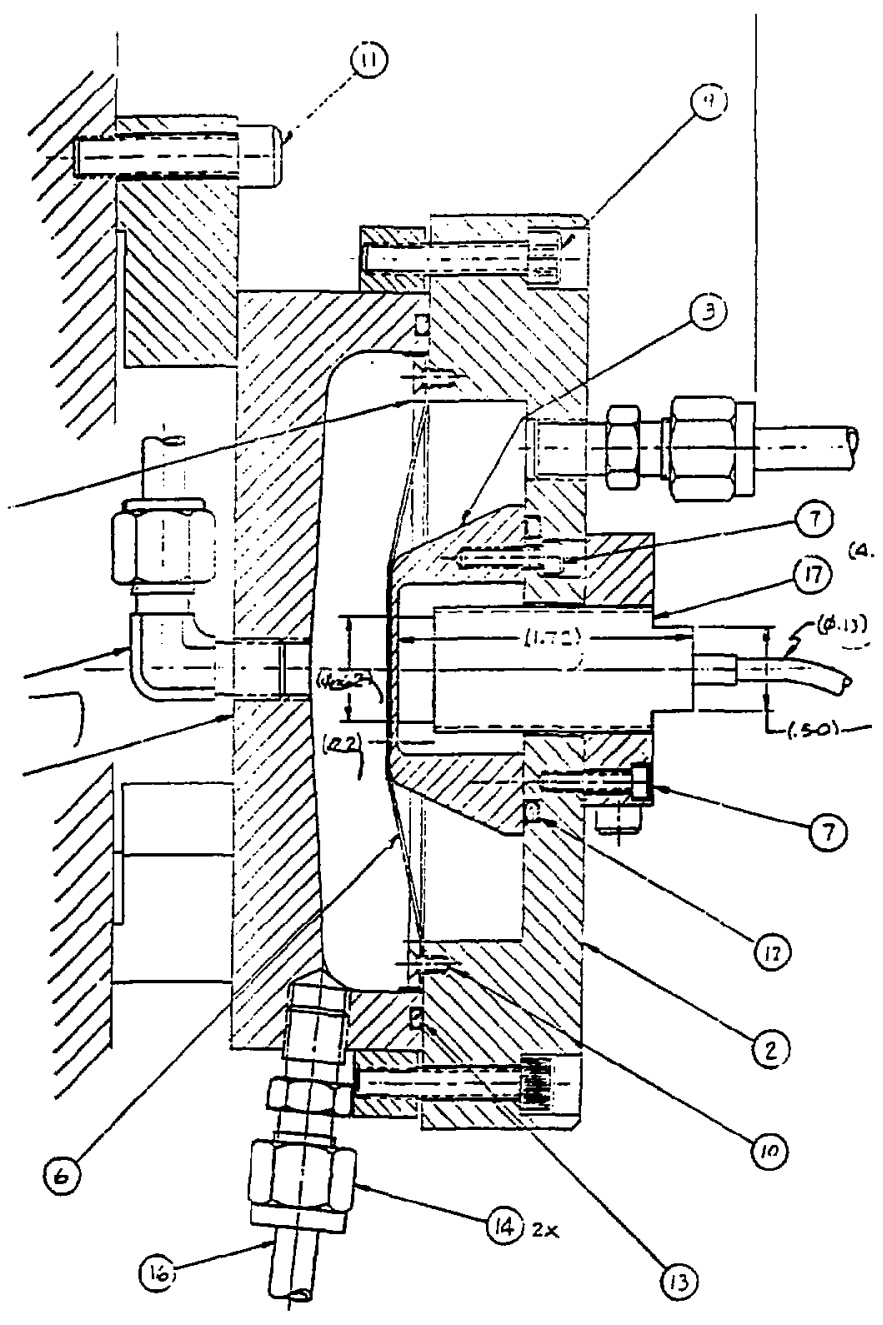

Figure VI.D.8. Assembly of precision mercury level cell. 
EW TILT IN MICROR DDLANS

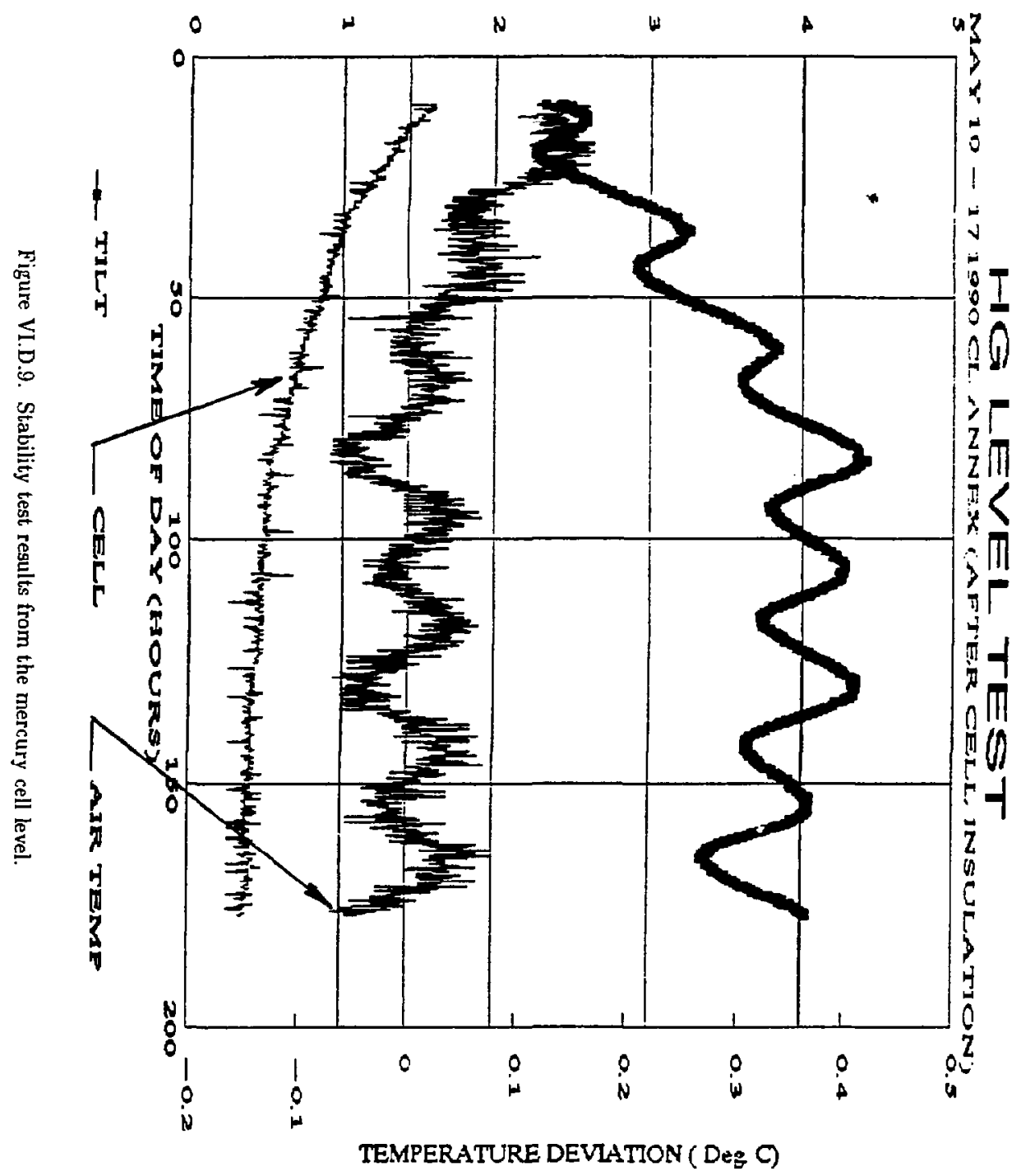




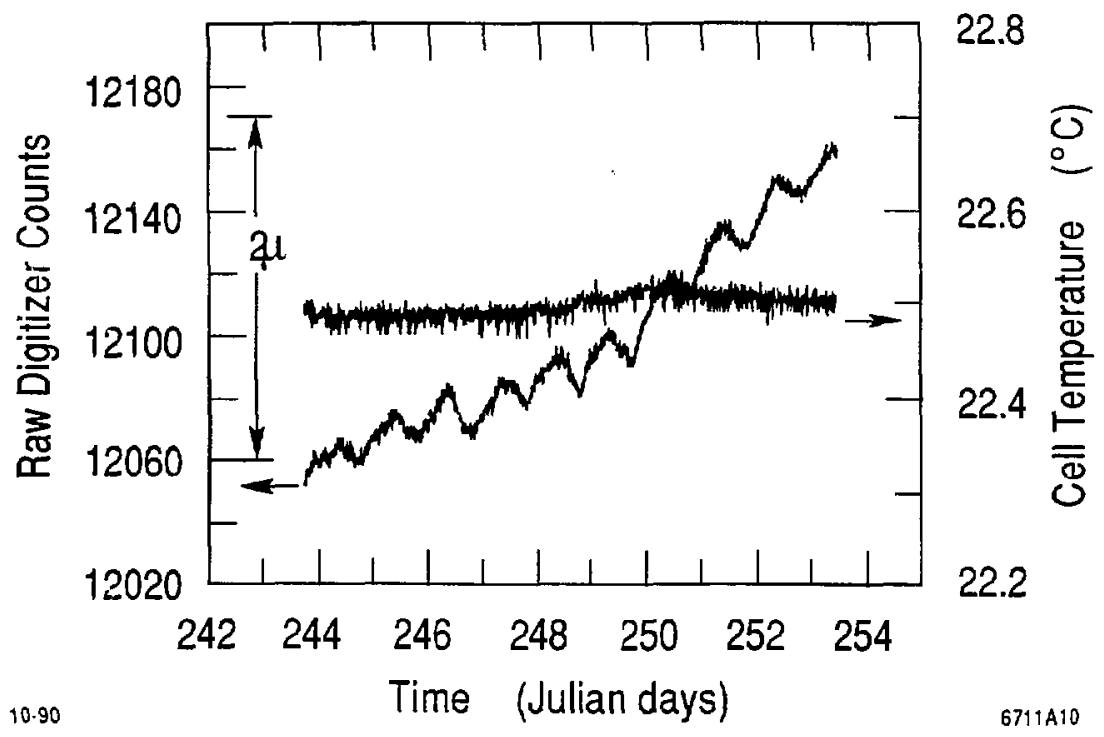

Figure VI.D.10. Sector 10 tilt stability results. 


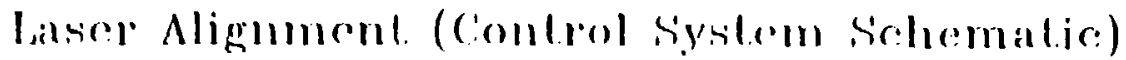

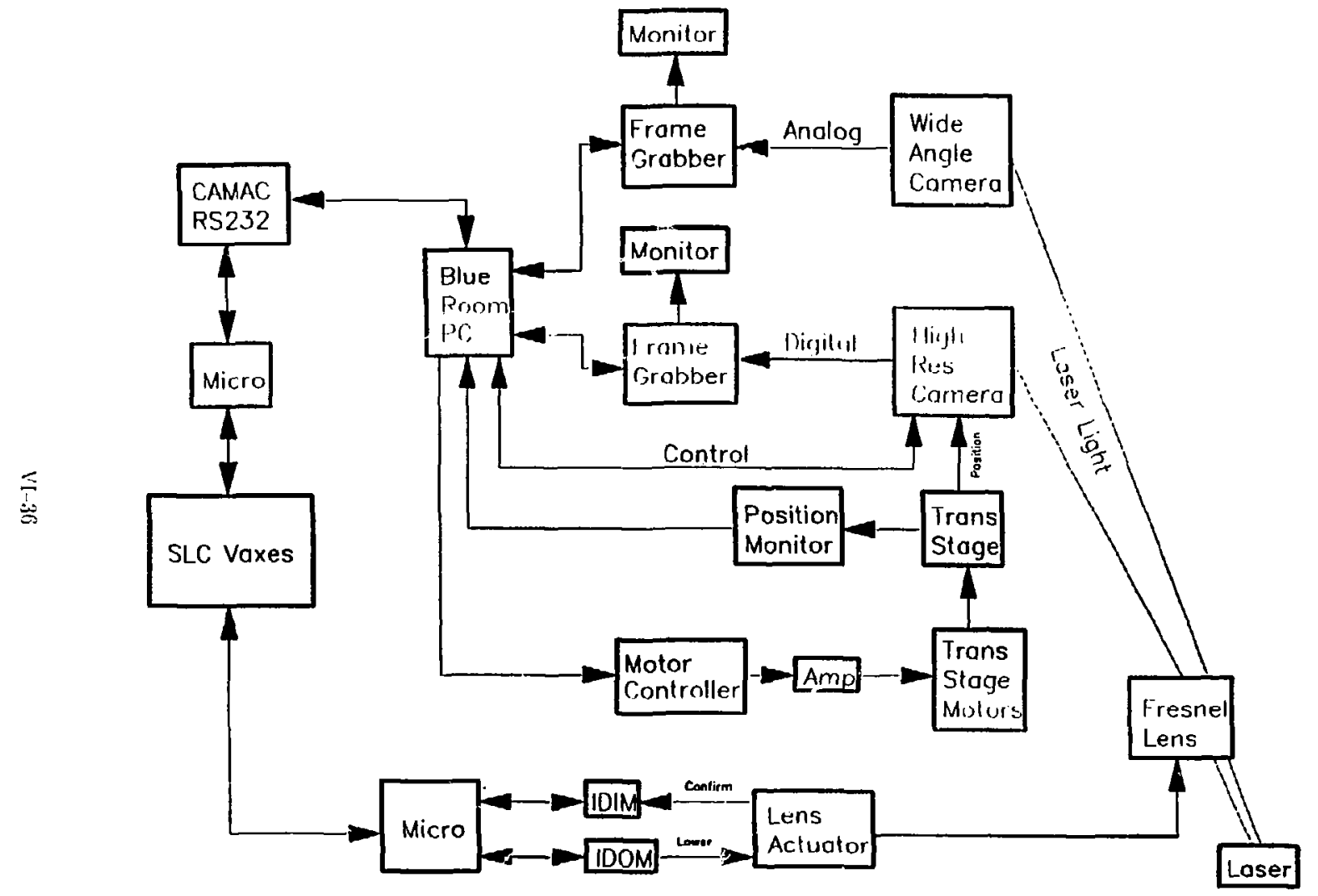

Figure VI.D.11.1. Schematics for the Integration of various Alignment Components into the overall Control System: Laser alignment. 
Tilt Measurement Device (Control System Schematic)

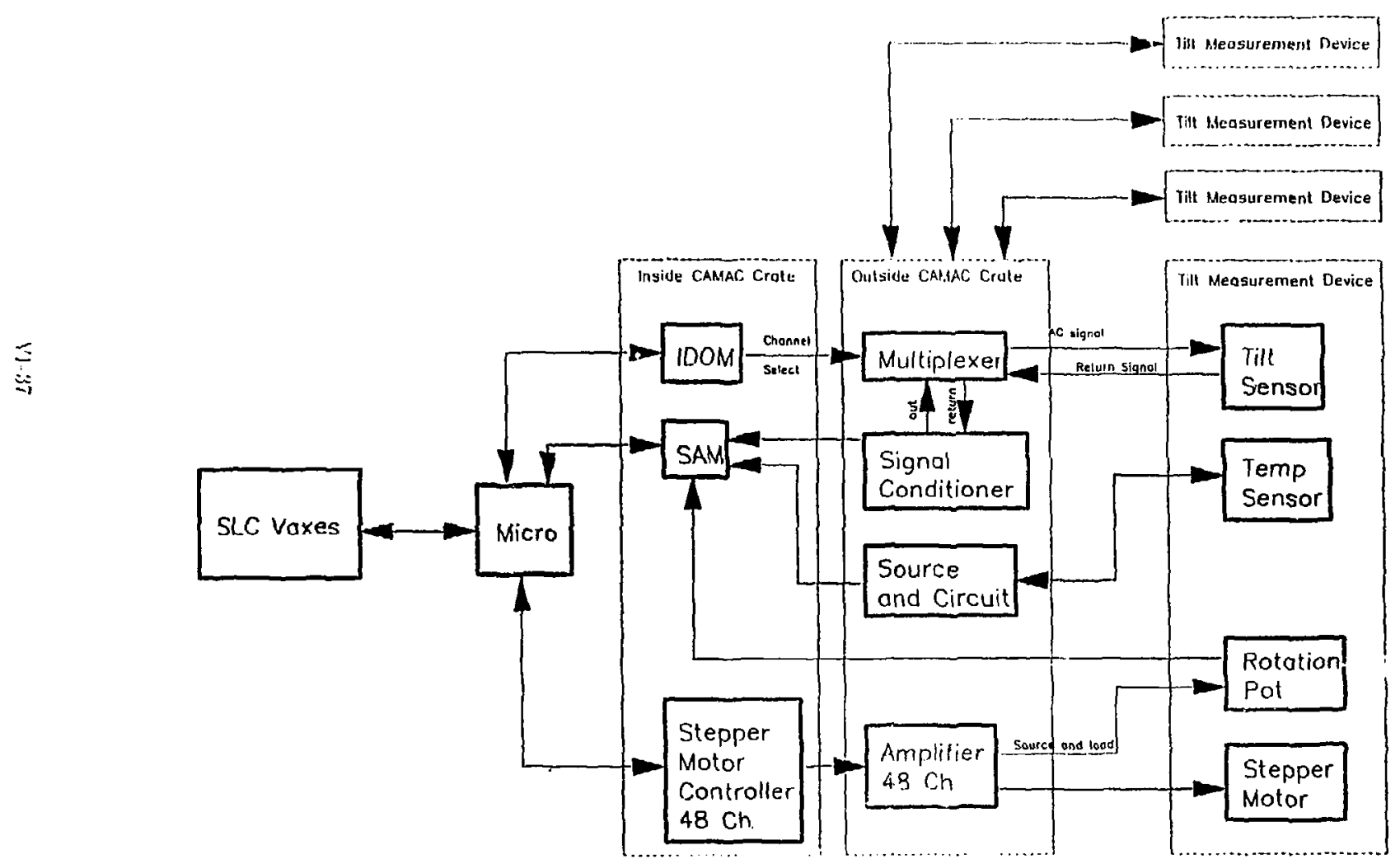

Figure VI.i 11.2. Schematics out the Integration of various Alignunent Components into the overall (Oontrol Systent: 7ill measurement device. 


\section{Proximity Sensor (Control System Schematic)}

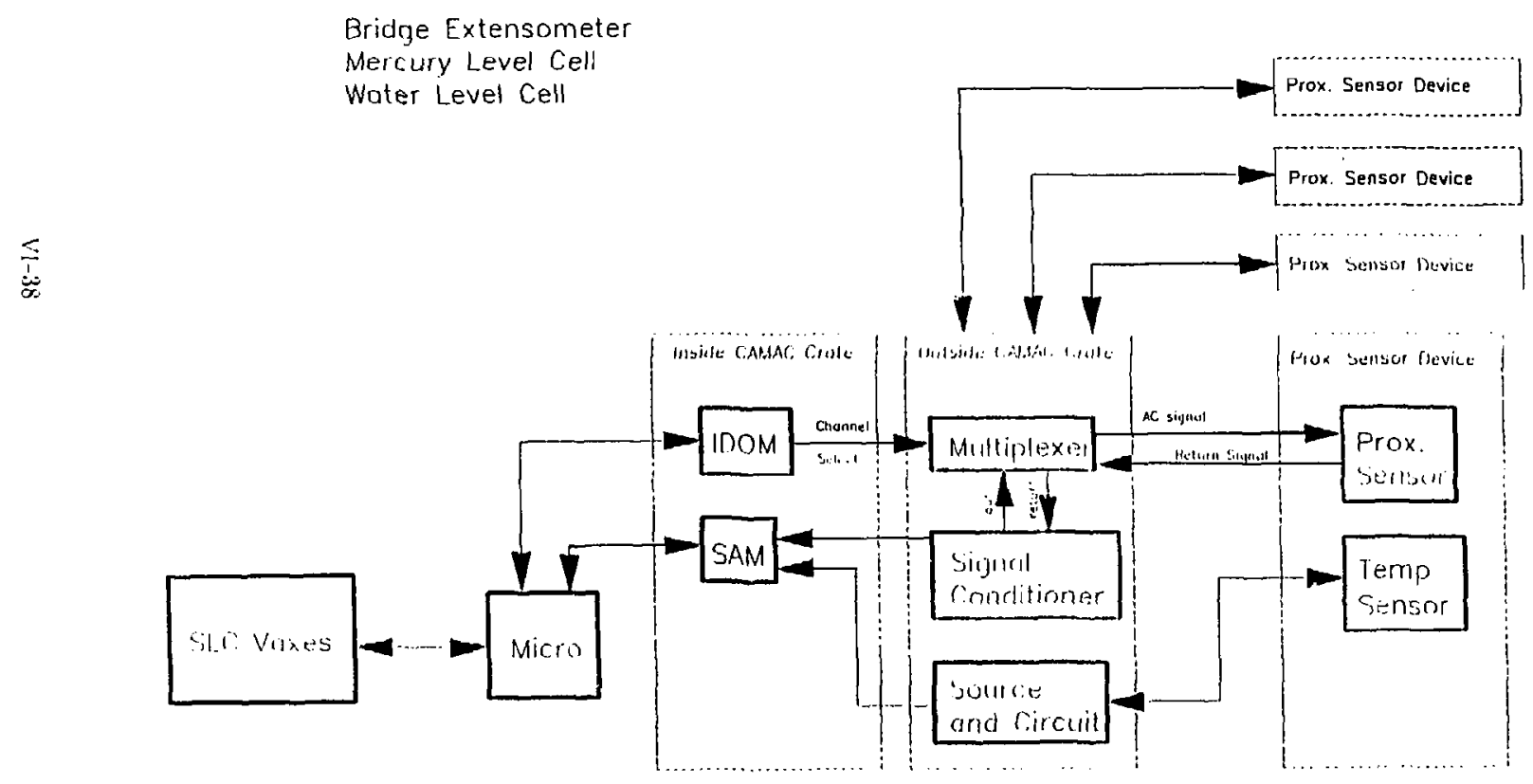

Figure VI.D.11.3. Schematics for the Integration of various Alignment Components into the overall Control Systern: Proximity sensor. 


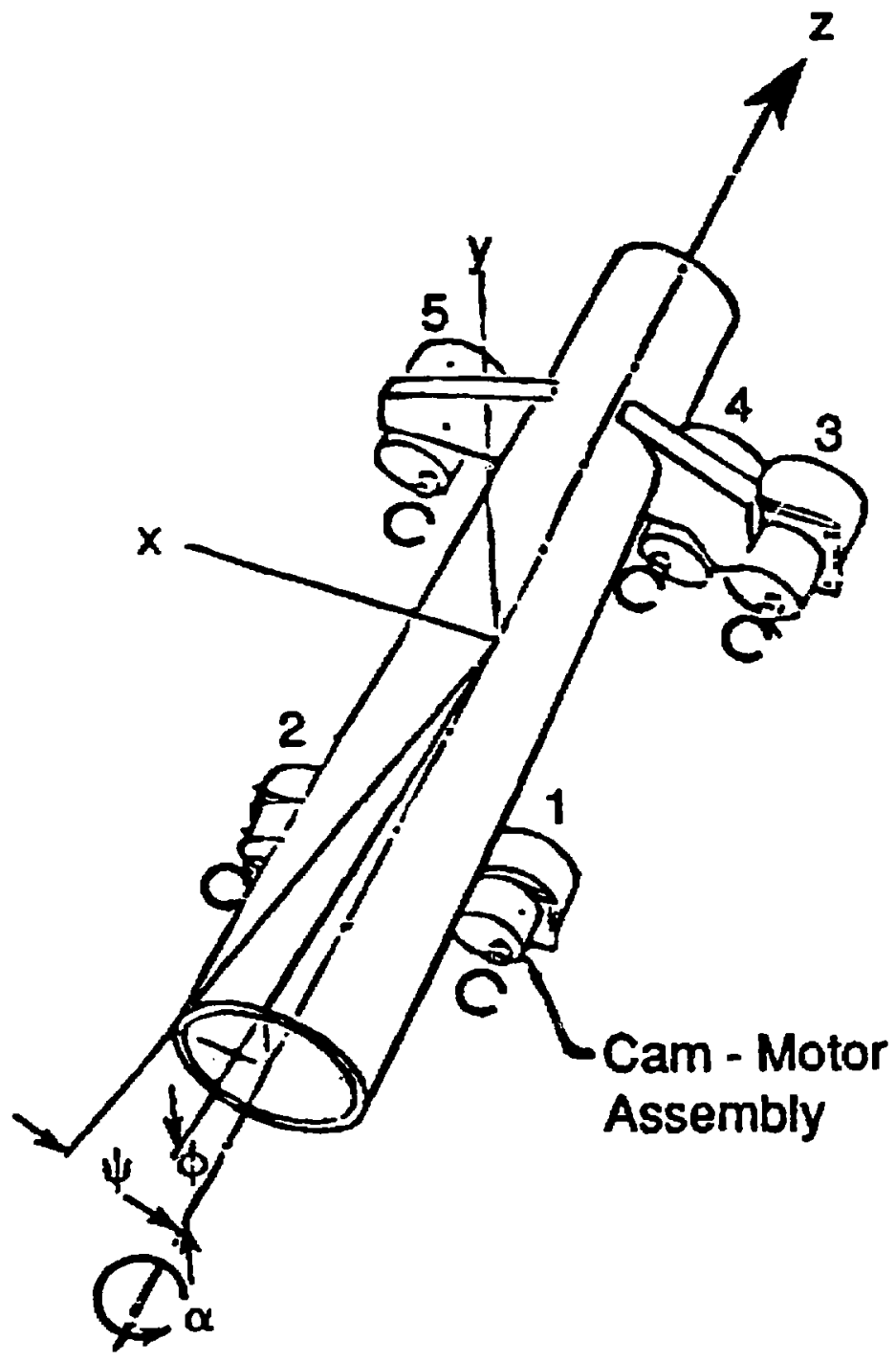

Figure VI.E.1. Sketch of a cam-operated actuator controlling five degrees of freedom. 


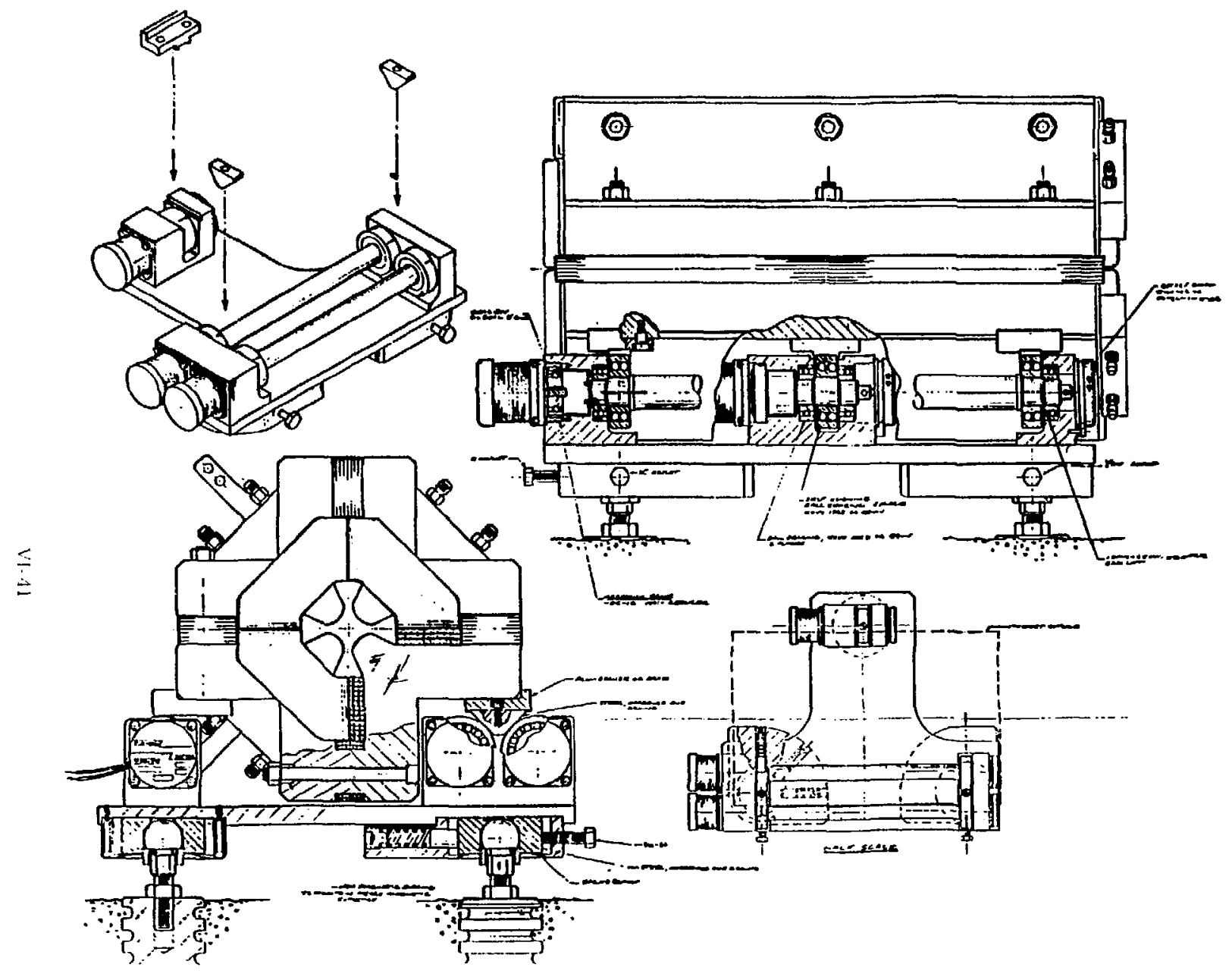

Figure VI.E.2. Assembly drawing of a cam-actuated magnet mover with three degrees of freedom. 


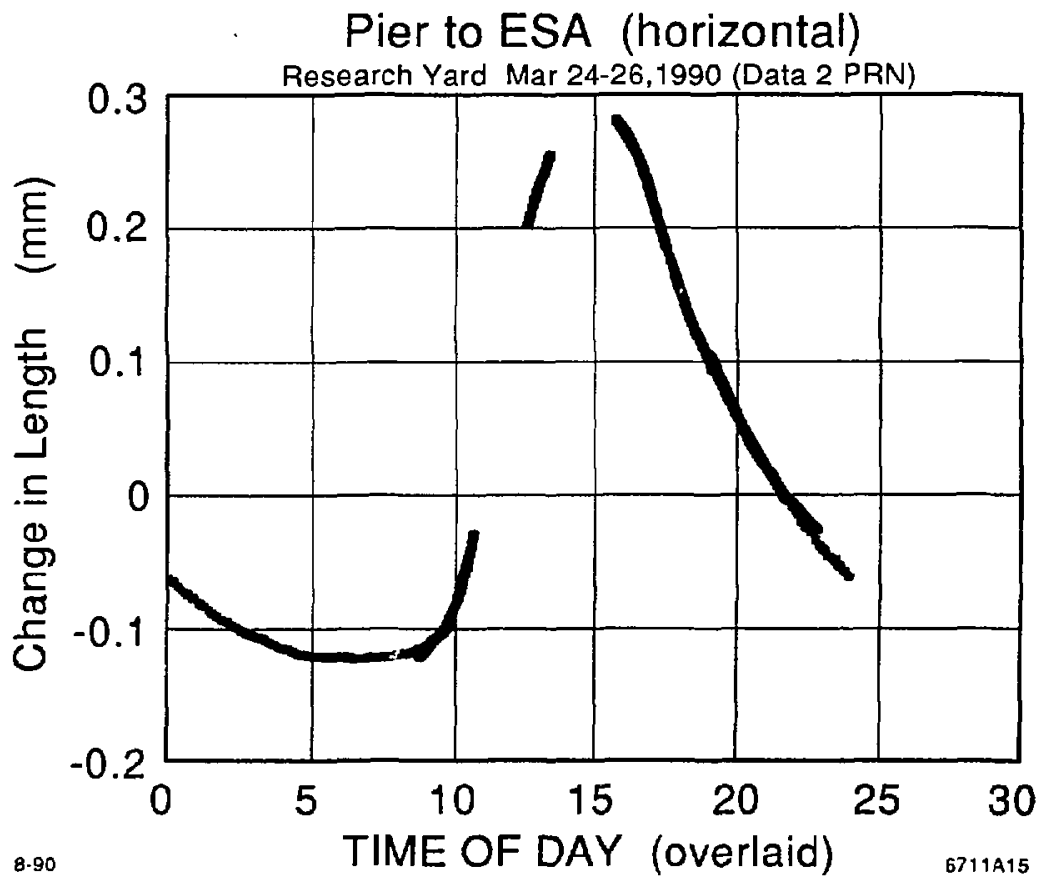

Figure VI.F.1. Change in the 33 meter distance between ESA and Pier $\mathrm{A}$ as a function of time of day. 
CHANGE NN DISTANCE (MM)

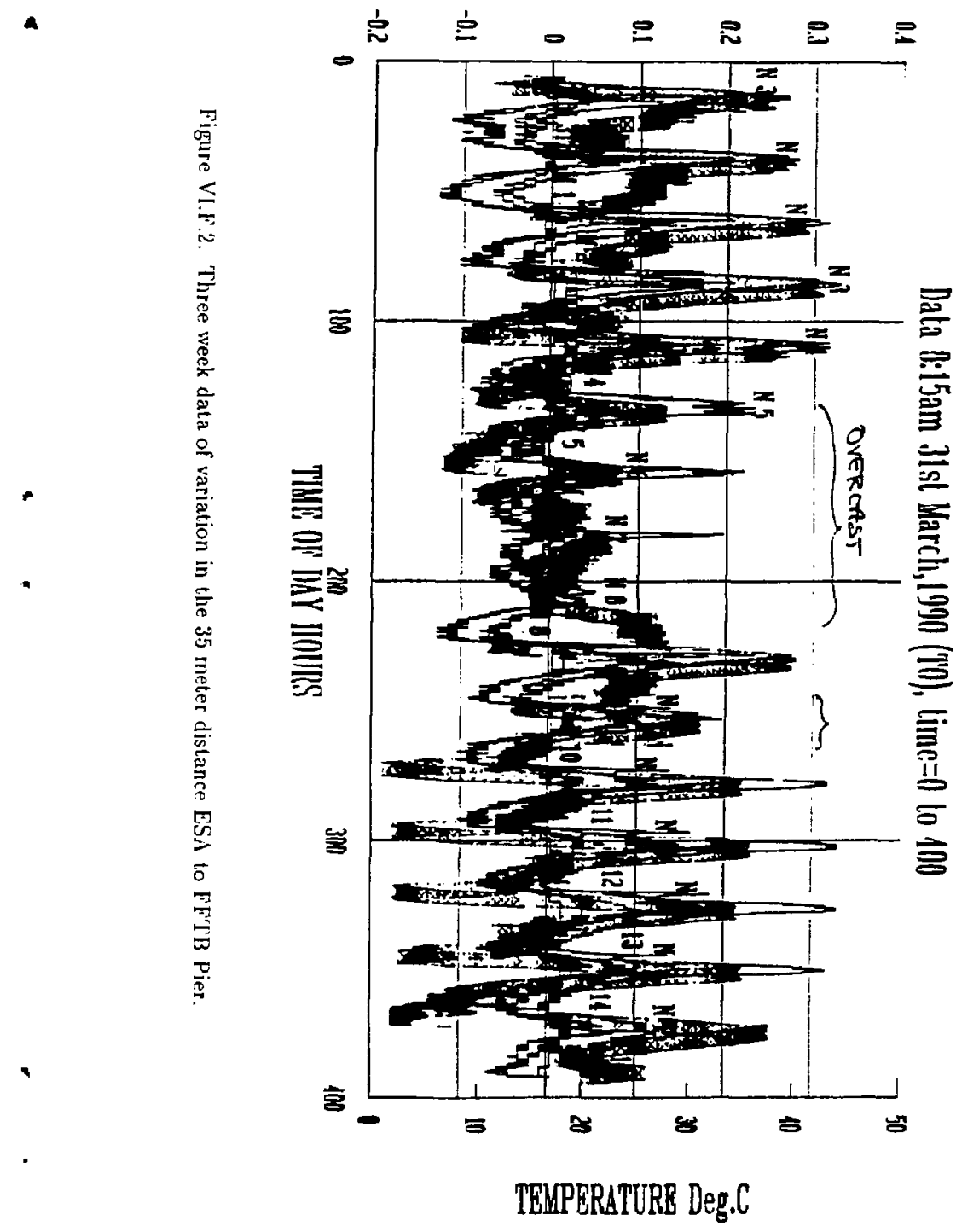




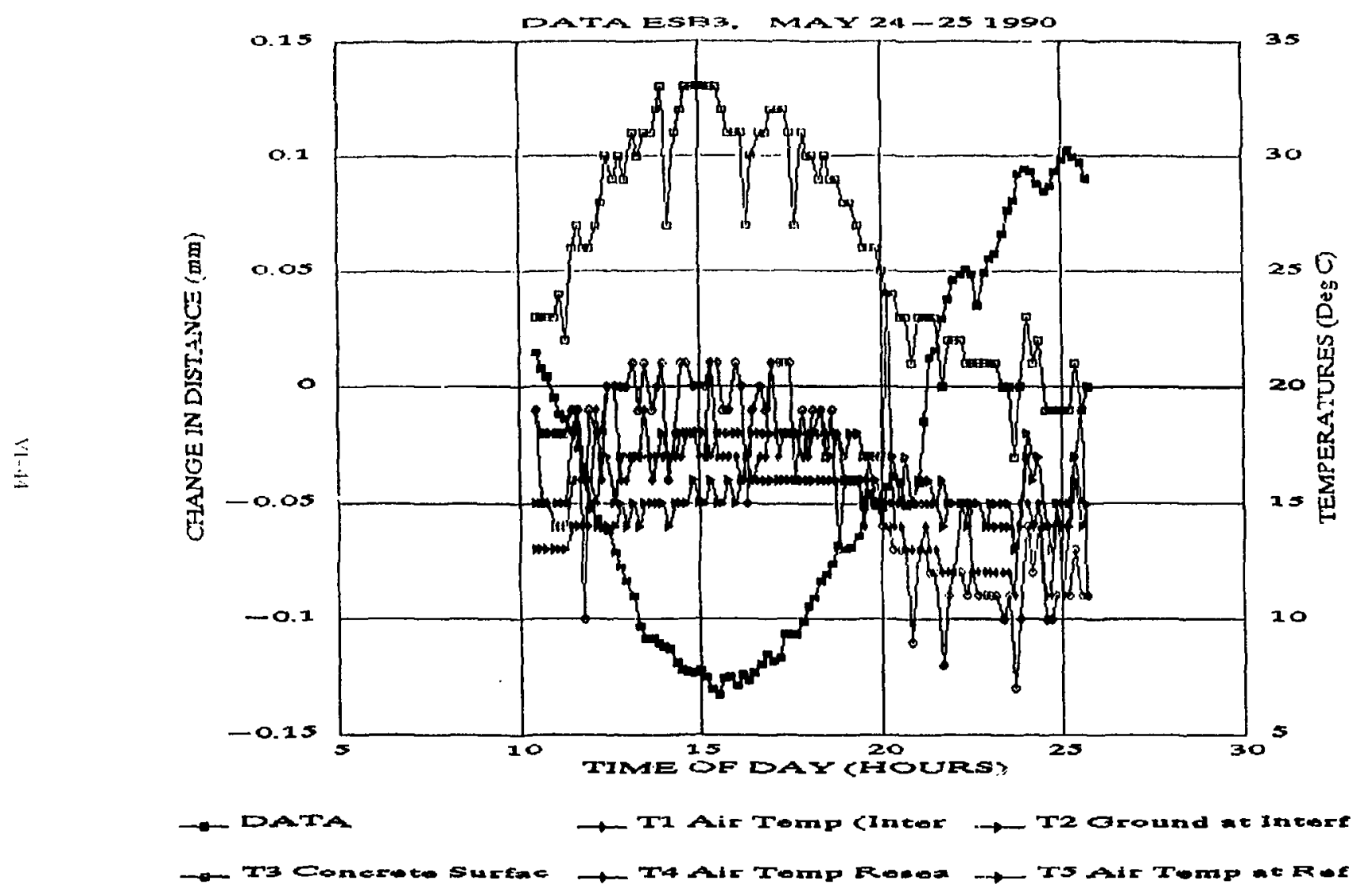

Figure VI.F.3. Variation in the distance from End Station B to Pier A 


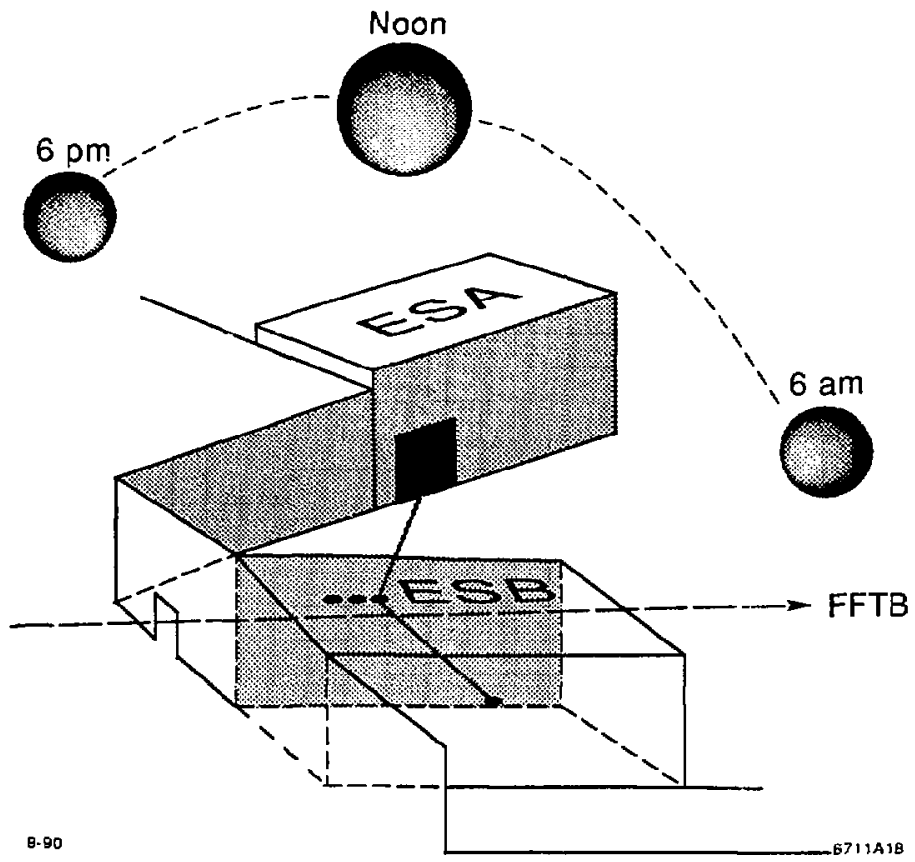

Figure VI.F.4. Differential heating of the research yard pad as a function of various azimuths of the sun cluring the clay: 


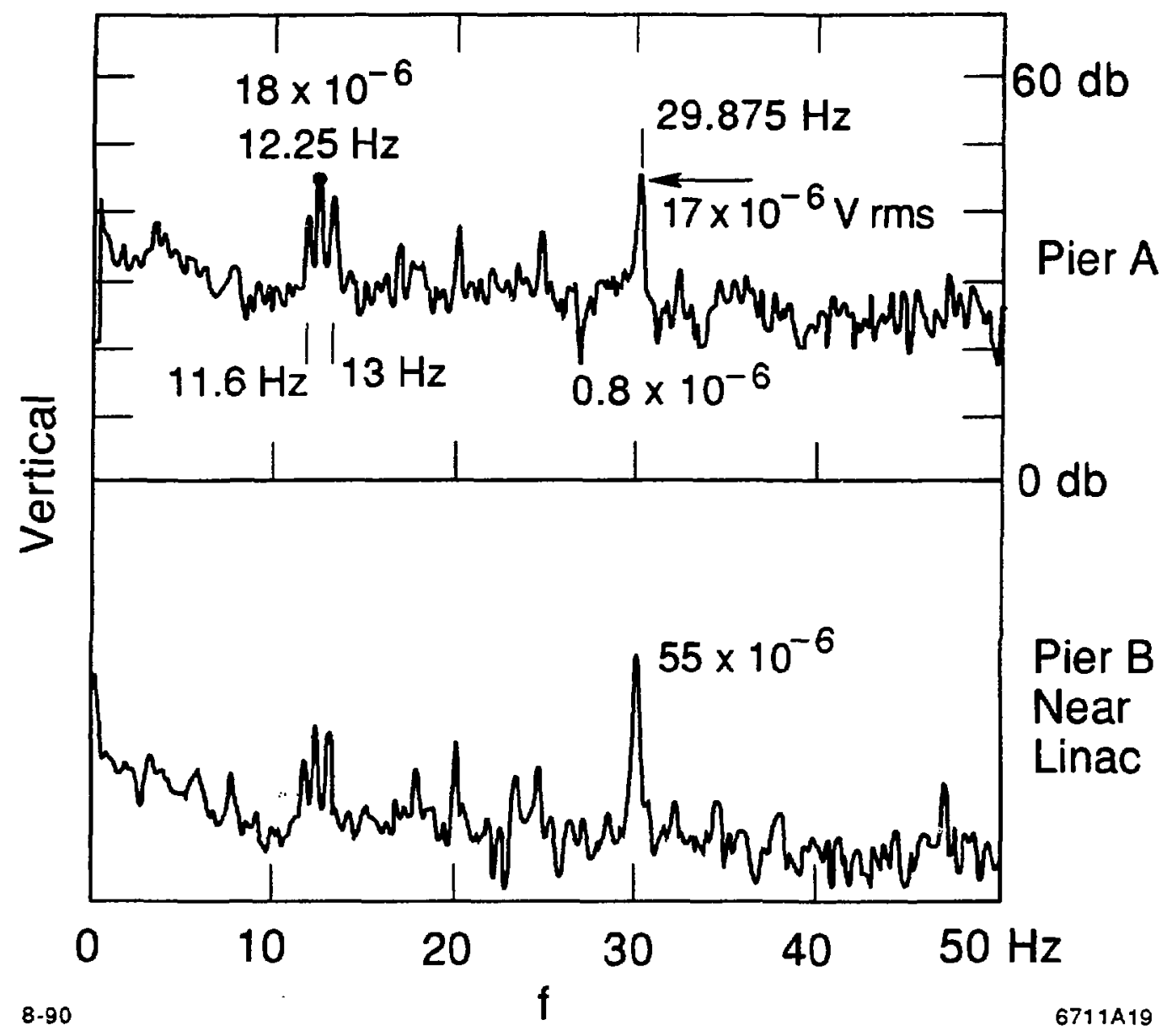

Figure VIF.5. Spectrum of vertical ground motion velocity on $P$ ier $A$ and $B$ of the rescarch yard taken on May 9il 1990 9:30 am. 


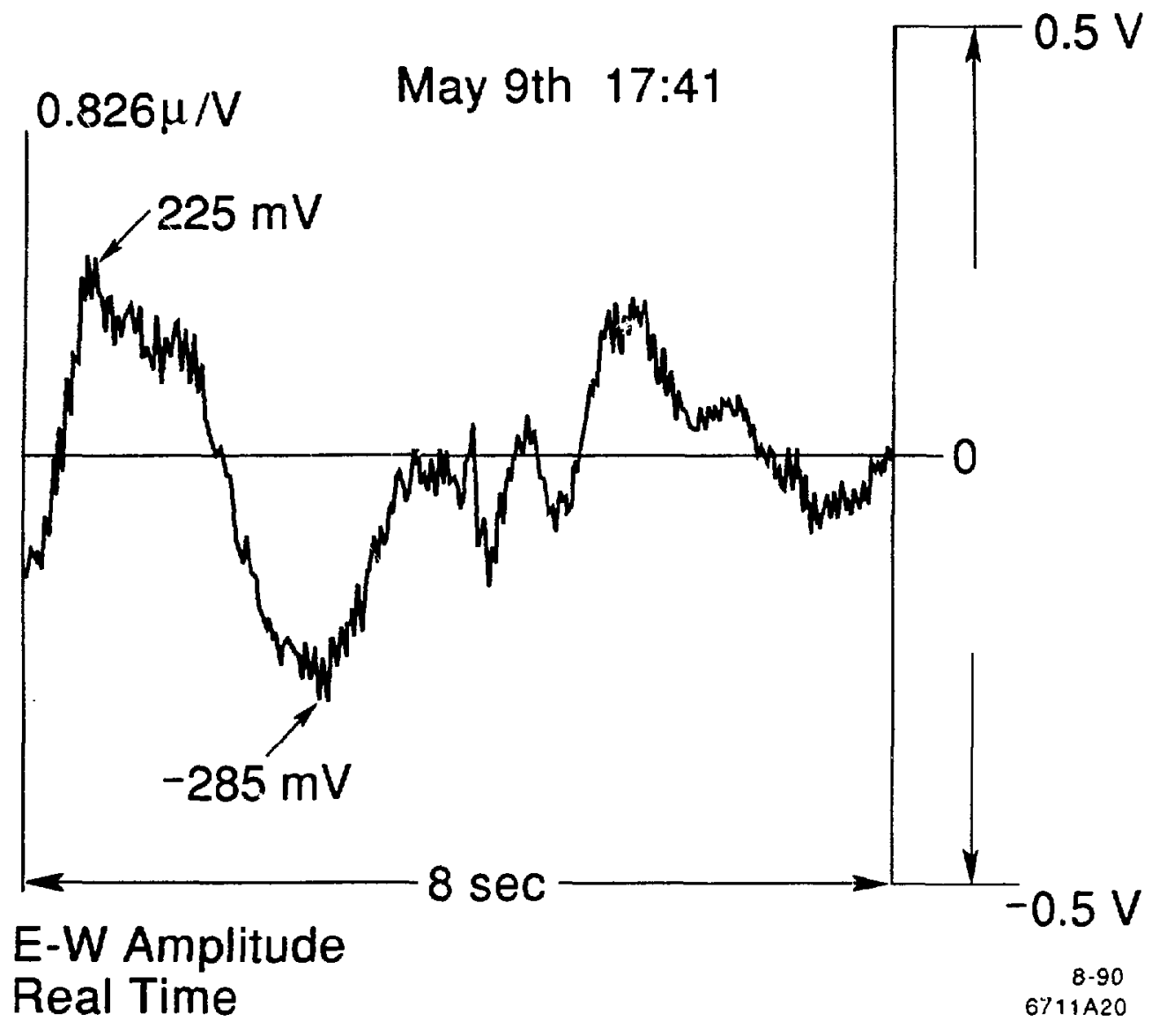

Figure VIF.6. Real time trace of E-W Horizontal amplitude. 


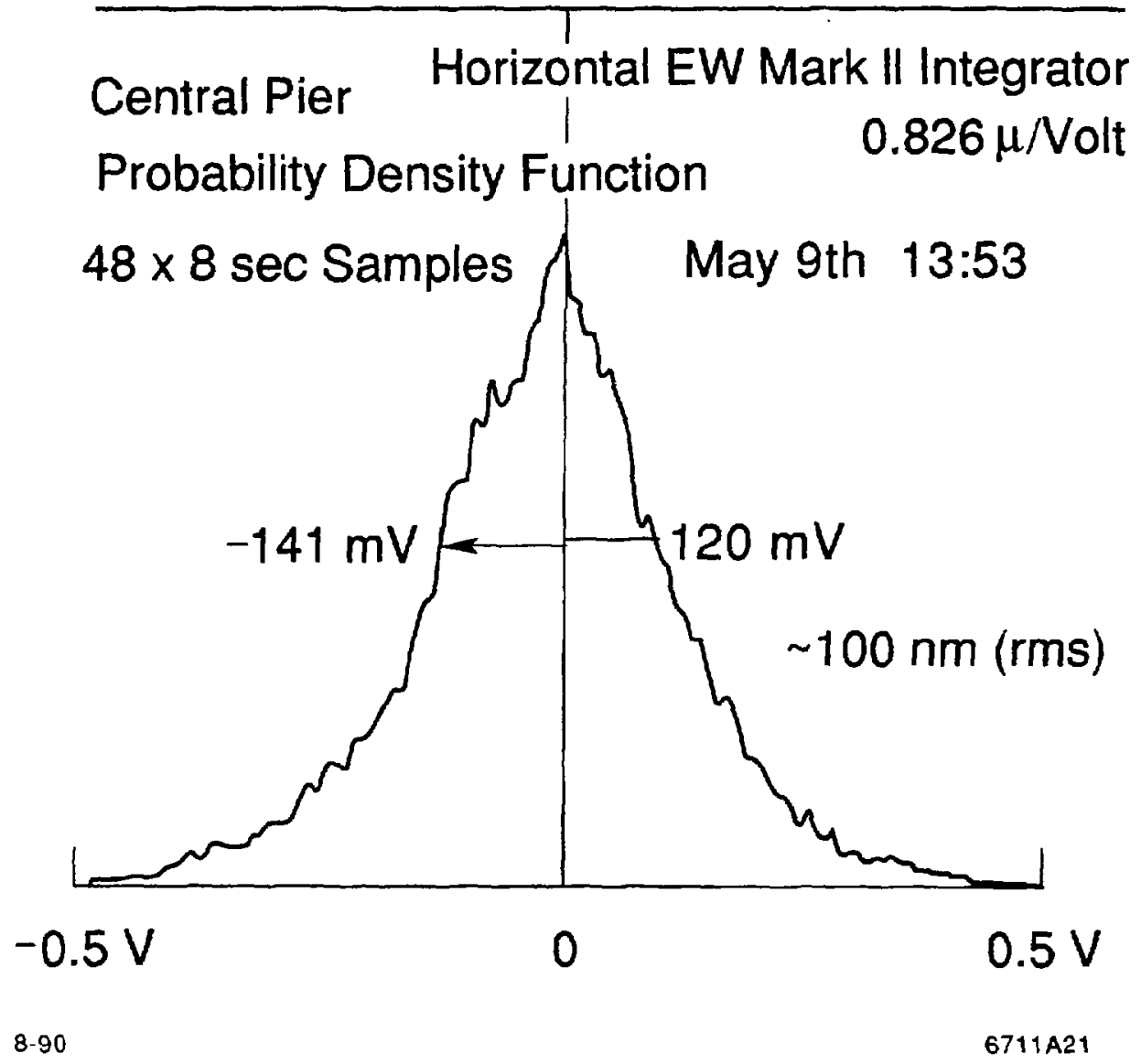

Fignre VI.F.7. Probability Density Function of Horizontal Motion (Windy). 


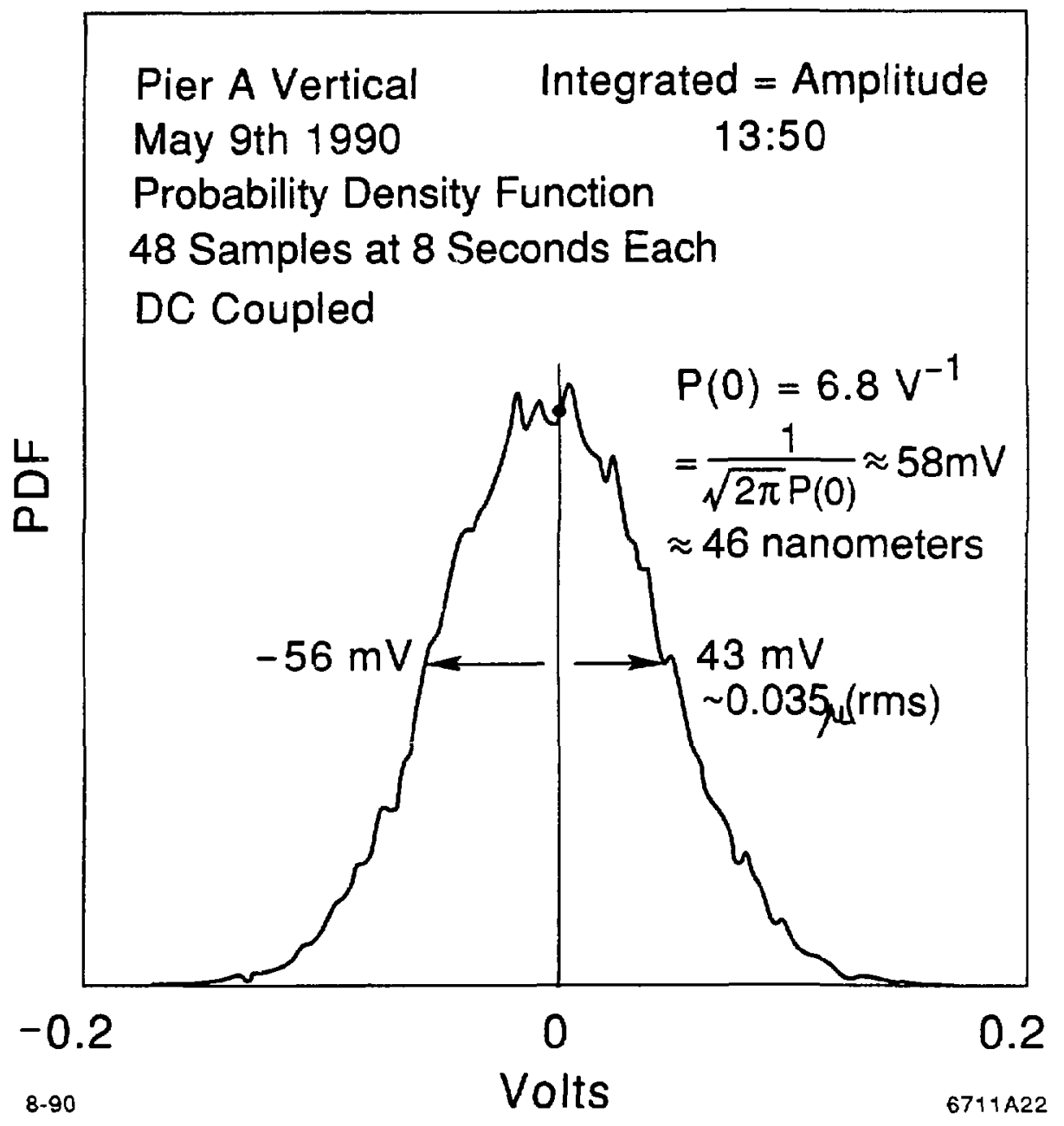

Figure VI.F.8. Probability density function of Vertical amplitude on Pier A. 


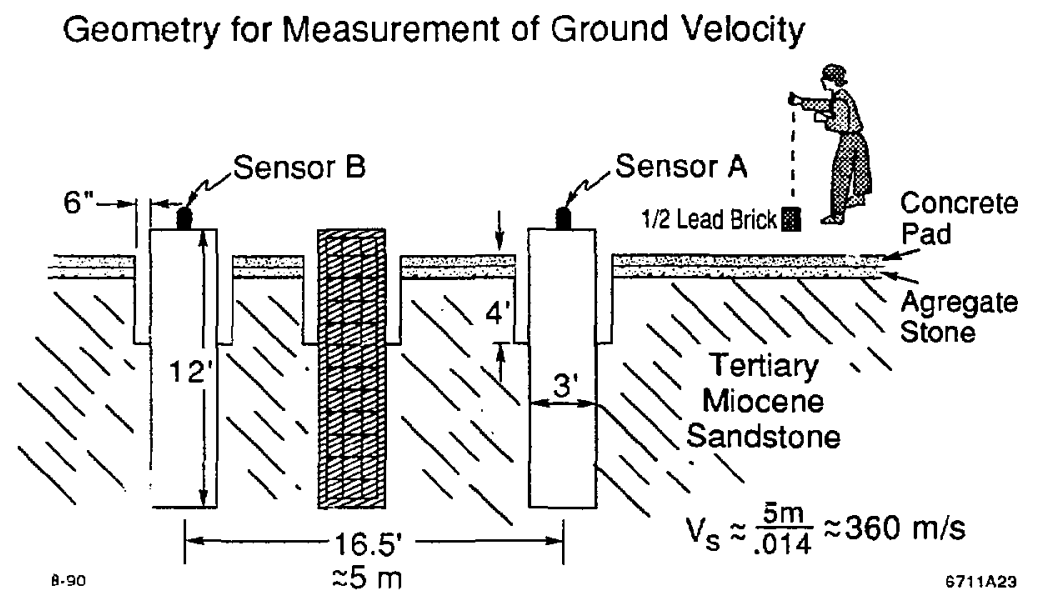

Figure VI.F.9. Geometry of the piers used to make ground velocity measurement. 


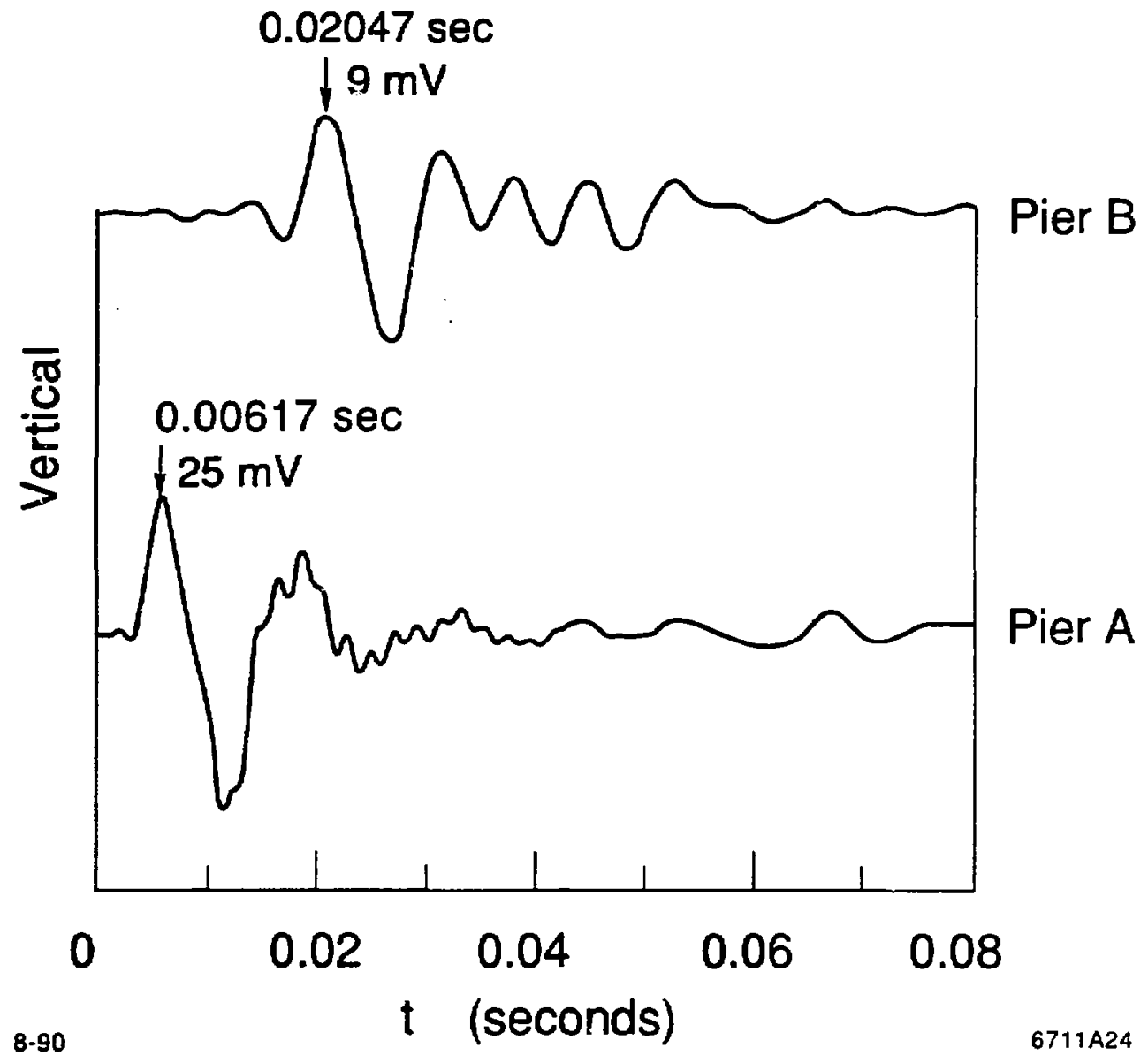

Figure VI.F.10. Display of time of atrival of sound on Piers $A$ and $C$. 
VII. Vacuum System 


\section{Vacuum System}

\section{VII.A Specifications}

Since the FPTB is not intended to produce beans for particle physics use, the vacuum requirements are not very sctere. It is necessary to maintain sufficiently low vacuum to allow the clean transport of the beam and to avoid interference with the diaguostic instrumentation used to measure the properties of the bean itself. The principle specifications are:

- pressure of $10^{-6}$ Torr or less.

- all metal, unbaked, $25 \mathrm{~mm}$ m 30.1 stainless stcel beam line using knife-edge type fanges.

- ion punps and hot filament gauge tubes used throughout.

- all controls in one location.

\section{VII.B Design}

The beam line is planned to be divided into five regions. Each region will be 30 to 60 meters in lengtli; small enough that racuum leaks can be quickly isolated and repaired, and also minimizing the impact of vents and pump downs. Regions will be isolated by pneumatic gate valves whicls will be remotely operated but will have local over rides. These valves will be interlocked to the vacuum gauge controllers and also fast sensors or McClure switches. Sensors will be located downstream from each valve.

In the first or switchyard region of bean line, a $1.100 \mathrm{li} / \mathrm{s}$ diffusion pump will be used to pump both the PFTB beam line and the End Station A bean line. Actual pumping speeds will be concluctance limited by the 50 mm aperture size of the switchyard beam line and thus smaller by a factor of 15 from the speed at the pump. Pressure requirements in this area are only $10^{-1}$ Torr so this specl will be adequate.

All subsequent regions will be pumped with $20 \mathrm{li} / \mathrm{s}$ sputter jon pumps distributed discrectly along the $25 \mathrm{~mm}$ bean line. As in the switchyard, the pumping speed of this 
line will also be limited by conductance and beam line pressuse will make a transition from switchyard vacuum to the design value of $10^{-6}$ Torr in the sccond region. Lower pressure in the interaction region will be achieved by reducing the distance between pumps.

All instrumentation and all controls will be located in a single rack at one location. Each region will lave a junction box for gauge tulses and a second junction box for pumps. This allows the option of adding move of either at a later date should racuum requirements change. 


\section{FFTB VACUUM SCHEMATIC}

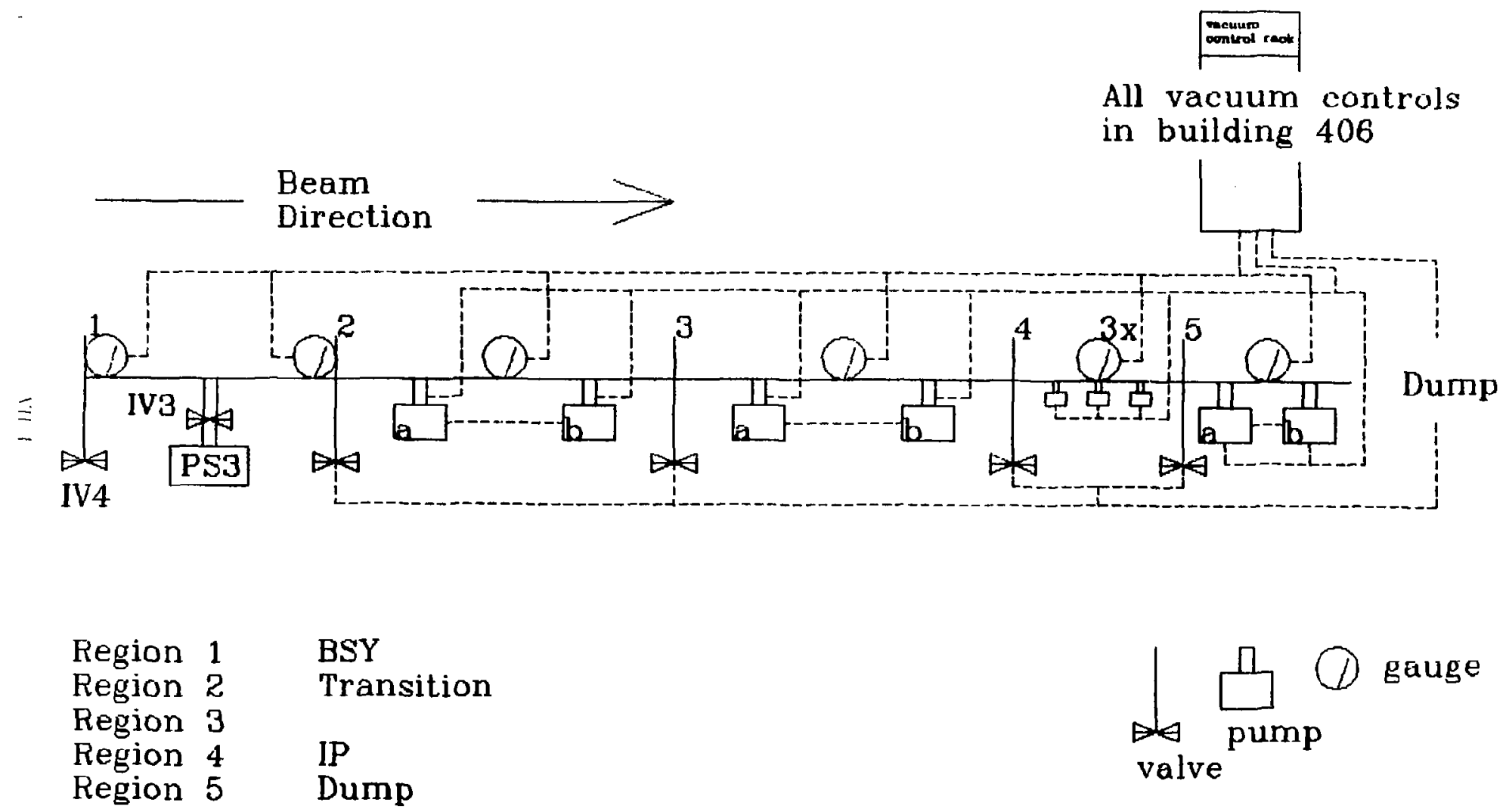




\section{Power Supplies}




\section{Power Supplies}

\section{VIII.A Specifications and Designs}

The tutal DC power requirements for the FF'B magnets. less than $500 \mathrm{~kW}$, is not large. but the need to control the strength of individual magnetic elements along the beam line dictiates that there be a relatively large number of power supplies. A total of 32 large supplies and 64 bipolar corrector supplies will be recuired to provide sufficient control to operate and tune the lattice optics.

The power supplies are grouped into four categories:

1. Higl power (greater than $10 \mathrm{~kW}$ ), conventional SCIR controlled, with stability of $0.01 \%$ of full scale (10E-4).

2. Ilighi power (greater than $10 \mathrm{~kW}$ ), high performance switclier supplies, with stability of $0.001 \%$ of full scale (10E-5).

3. Hedium power $(10 \mathrm{~kW})$ switcher supplies. with stability of $0.001 \%$ of full scale (10E-5).

1. Bipolar corrector power supplies (200 Watt). with stability of $0.005 \%$ of full scale $\left(5^{\times} 10 \mathrm{E}-5\right)$.

Wr will discuss each of these in turn.

\section{IIigh Power Conventional SCR Supplies}

'lliese supplies will be used witl five naguet groups, all clownstream of the Focal l'oint where the accuracy requirements are not too stringent. These magnet groups are lisicl in lable VIII.1.

Each power supply will deliver $40 \mathrm{kll}$ at cilher 500 or 800 amperes. Input power is 480 Volts AC. Output is controlled by means of SC'Rs, and the DC output is filtered. linits are air cooled, and are built in self-contained floor-mounted enclosures.

The installation of these supplies will loe sinular to that of existing supplies on the SLC. SLAC designed magnetic amplifier typo lanseluctors will be used to measure the J) C current, and regulator/rontroller chassis for each supply will be mounted in racks. 
Long-term stability in the curtent of $0.01 \%$ of full scale should be aclequate for these magnets. Power line fluctuations will cause occasional short-term transient excurtions as large as $0.2 \%$, but will not affect long-tern stability. All fire power supplies will be located in Bldg. 406.

\section{High Power Switchers}

There are five magnet groups (Talsk. Y $\{11.2$ ), all upstseam from the Focal Point. that have very stringent requirements, for which conventional S(R controlled supplies: would be inadequate. In these instances the plan is to use commorcially available highfrequency and high-power switchers to regulate magnet curents.

Each supply will deliver $50 \mathrm{~kW}$ at 125 rolts and 400 amperes DC. Eacls switcher will require a separate source of DC power, which will be a $60 \mathrm{kll} / 150 \mathrm{~V} / 100 \mathrm{~A} \mathrm{DC}$ SCR controlled power supply; of a type similar to that described in the previous section. The switcher assemblies will be purchascd to performance specifications from outside vendors, and will be installed in racks. Magnet current will be measured by means of two zero-flux DC transformers (DCCT). There will be a regulator/controller chassis for each circuit. All five power supply systems will be located in MCC.

Details that explain the choice of these high power switchers are covered in a later section. The goal will be to obtain both long-term and short-term stability in the oulput current of $0.001 \%(10 \mathrm{E}-5)$.

Trim windings on each of the lour poles of tnagnets that are ganged with other magnets will be brought out separately. These can be connected in various configurations to corector supplies to permit trimming of individual maguets.

\section{Medium Power Switcher Supplics}

There are a total of 18 magnet groups. mostly quadrupoles, where this type of power supply will be used. Table VIII.3 lists these magne.1 groups along with the location of the corresponding power supply.

Although the required currents dillen considerably between magnets, one common power supply size was chosen to provide sarings in operation and maintenance. For the same reason $Q(: 3$, rather than laving a single large power supply unlike any other 
will be operated from two swilcher supplies connected in parallel. QP4 and BA A/H wit! also use this type power supply, even though they are downstream of the Focal Point and thus have less stringent stability requirements.

Details that explain the choice of switchers are corered in a later section. The goal will be to obtain both long-tern and short-term stability in the output current of $0.001 \%$ $(10 \mathrm{E}-5)$.

Bipolar Corrector Poier Supplics

This group includs's all the power supplies that arr required to feed steering magnets, (quadrupole trim and/or stcering windings, and small magnets that are part of the beam launch group. Stewing and corrector magnets generally must be controlled around and through the zero point, hence they recuire bipolar power supplies.

There will be a total of 6.1 corrector supplies, $18 \mathrm{in} \mathrm{MCC}$ and 16 in Building 406. Each corrector supply will be rated 200 Walts \pm 20 rols DC \pm 10 amperes DC.

A design dercloped by CEBAF and also used on the SSRL 3-Gev Synchrotron will be followed for the corrector power supply systen. In this approach the individual correctors are clesigned as plug-in modules, with eight modules to a crate. Three such crates will be mounted in a single rack, together witl clistribution panels, individual circuit isolating fuses. and two $2_{2} \mathrm{VDC} / 250 \mathrm{\Lambda DC} / 6 \mathrm{~kW}$ power supplies that provide plus and minus power to all the correctors in a rack. There will be two such racks in MCC, and one in Bldg. 106, with the lat ter licving expansion capabilities for adding eight more corrector channels at a later clate, if needed. The bipolar supplies are linear regulators, hener dissipate consiclerable powrer. With as much as 60 Watts per unit, the worst case dissipation in a rack conlel reach $1.5 \mathrm{k} / \mathrm{V}$. Since the morlules are air cooled, as are the DC: bulk power supplies, it will be necessary to lorce rentilate each rack.

C'turent measurement and current regulation is doue by means of current measuring resistors installed in each crate. With this medhorl. and in an air conditioned environment, it should be possible 10 achieve stable operating conditions to within $0.05 \%$ of maximum current rating (5 milliarujeres). 
Control and readout of the correctors will be by means of DA and SAM (sample and hold) modules in a CAMAC crate. An alternative being considered is the addition of power supply controller modules as part of cach corrector plugin unit. These controller modules perform the necessary DA and AD functions, and link to the CAMAC crate via a serial link. This latter technique was developed for CEBAF by an outside vendor, and eliminates sending analog signals over long clistances, as well as simplifying cable interconnections.

\section{VIII.B Comments About Switcher Supplies}

Measurement and control of current to an absolute accuracy better than $0.01 \%$ is extremely hard to achieve and to prove. On the other hand, obtaining currents that are stable, and being able to return to a desired setpoint with a precision of $0.001 \%$, is a realistic goal, though difficult and expensive. It should he achicvable with the type of equipment that will be used for the power supplies that foed magnets upstream of the Focal Point.

The first requirement is the ability to measure the current with the desired precision and reproducibility. Zero-flux DC Current Transformers (DCCT), though three times more expensive than the traditional magnetic amplifier transductors used at SLAC thus far, will be used for the FFTB. A number of DCC'Ts are now being evaluated at SLAC'. and have been used extensively at CERN and PESY in the recent past. DCCTs have the advantage over shunts in that they provide a galvanically isolated large signal (up to 10 volts) without dissipating a large amount of power. Zero-flux DCCTs have the further advantage over the magnetic auplifier type in that they are linear and relatively insensitive to line voltage fluctuations.

Two DCCTs will be installed in all switcher power supplies where stability and precision of $0.001 \%$ is required. This redundant current monitoring, though expensive.

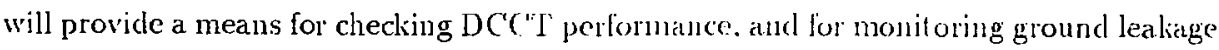
currents, since the wo DCCTs will be placed one on the positive. the other on the negative feeder lines to a magnet. Any discrepancy will be indication ol rither DC('T malfunction or of ground cursest. 
The second requicement is for a fast power controlling device. Phase controlled SCRs that rectify $60 \mathrm{~Hz} A C$ power are the traditional workhorse for large power supplies because of their reliability and efficiency. linfortunately it is practically impossible to build SCIR supplies that will not undergo a temporary excursion in the output when there is a transient change in the line voltage. Although long-term stability of $0.001 \%$ is achievable for SCR supplies, short-term excursions that exceed this value are bound to occur in all practical installations, except for magnets with extremely long $L / R$ time constants. The FFTB quadrupole magnets are relatively small and operate at room temperature, hence will probably have time constants of less than 0.5 seconds. This is too short to meet the stringent recuirements using SCR controlled supplies. For this reason switcher supplies will be used.

Switcher teclinology has come into its own the last five years, and power supplies rated $10 \mathrm{~kW}$ are now conmercially available. Switcher supplies rely on rather high flequency operation (up to $80 \mathrm{KHz}$ ) of power switches in which the average output is controlled by means of pulse width modulation (PWM). The output of the supplies is filtered to prevent if interference. The advantage of switchers is speed of response, thus permitting the design of a regulator in which the effect of transients can be minimized. Switcher supplies, just as SCR supplies. but unlike power supplies that use series transistor banks for controlling the current, are efficient clevices. Hence it will be possible to air cool, rather than having to water cool, the supplies.

Switcher power supplies will be purchased to performance specifications from outside vendors. The internal circuits of these power supplies will not be used for current regulation. Instead. the supplies will be used as high speed power amplifiers that will respond to an amplified error signal that will be generated in a separate SLAC designed regulator/controller chassis, in which the signal from a DCC T will be compared with a reference signal generated by a 16 -bit DA converter. For this reference a digital rather than analog link to the remote input from a CAMAC crate should avoid thermal emf's and noise pickup. The regulator/controller chassis will also contain the necessary magnet interlock supervisory circuits, on-of control. computer link, and will generate warning and PPS signals. 


\section{VIII.C Support Facilities}

The power supplies will be installed in two locations. (See also Section XI of this report.) Building 5, the Accelerator Main Control Center (MCC), will house all the supplies that feed power to the magnets inside the Beam Switchyard. Room for this equipment and 480 volts $A C$ and 208 volts $A C$ power will be made available with the removal of the SPEAR transport power supplies and other equipment that is no longer in use. The East end of the building, where this equipment will be located, will lave to be air conditioned to within $5^{\circ}$ in order to help meet stringent stability requirenents, and to remove the heat generated by the air cooled power supplies. Building 406 in the Research Yard will be moved to a new location next to the bam line. and will house all power supplies that feed magnets outside the Bean Switchyard. This building also will have to be air conditioned to within $5^{\circ}$.

Power supplies for the magnets of the final lens doulblet (QC:2, QX1, and QC1) wil] be designed and fabricated at KEK. The power supply for $Q(12$ is rated $1.50 \mathrm{~kW}$, and will be the largest power supply in the entire FFTB complex. These three are the only power supplies that will require water cooling. These supplies will be installed in Bldg. 406.

Typical magnet currents are on the order of a few hundred amperes for the large magnets, and less than 10 anperes for trim and steering windings. Insulated air cooled cables installed in trays will be used to comect power supply DC output to the magnets.

Magnet protection, interlock and warning circuits will be sinilar to the type now in use in the switchyard. In this scheme all exposed nugnet terminals are covered, and individual magnets are provided with a safety box that is provided with a "Run-Safe" switch that can turn off the power supply: and a red flashing light that warrs anyone in the vicinity when that magnet is energized. The flashing lights will be turned off whenever the switchyard or tunnel are locked and in the "Lo Access" mode. to prevent. interference from the flashers. The magnet safely boxes will also serve as the junction points to which magnet temperature and water flow swit dess are connected for hookup to the power supply interlock systems. 
Table VIII.1 Magnet Groups With Large Conventional Supplies

QP1A and QPIB

QP2A and QP.2B

$Q P: 3 A$ and $Q P: 3 B$

QP5

$B 05 A$ and $B 0.5 B$

Table VIII.2 Magnet Groups With Large Switcher Supplies

\begin{tabular}{ll}
\hline B1 and B2 & string of 12 magnets \\
QN1 and QN2 Group 1 & string of 4 magnets \\
QN3 Group & string of 4 magnets \\
QN1 and QN2 Group2 & string of 1 magnets \\
QN3 Group & string of 3 magnets \\
\hline
\end{tabular}


Table VIII.3 Magnet Groups Witı Medium Size Switcher Supplies

\begin{tabular}{ll}
\hline MCC & Building 406 \\
\hline Q5 & QC4 \\
Q6 & Q1'. \\
QA0 & B3A and B3B \\
QA1 & B4A and B1B \\
QA2 & SF1A and SF1B \\
QT1 & SD1A and SD1B \\
QT2A & QC3 (2 supplies) \\
QT2B & \\
QT3 & \\
QT4 & \\
QC5
\end{tabular}




\section{Control System}




\section{Control System}

Operation of the FFTB beam line and decectors will be done from the SLC: Main Comtrol Center (MCC) where it will be possible to monitor and contral the upstream portions of the machine as well as the FFTB itself. Touch-panel consoles located in MCC provide the human interface to the machine hardware. For commissioning and maintenance purposes, additional control terminals will be built into the electronics racks in the FFTB support buildings. These local terminals provide control of the hardware, but lack the graphics capabjlities of the MCC consoles.

Control of the FFTB hardware. and acquisition of data from the sensors and instrumentation in the beam line, will be done by a CAMAC-based system that is an extension of the existing SLC control structure. The FFTB hardware is designed to be compatible with the SLC database and protocols, and will require little new software to operate. The full compliment of on-line analysis and modelling programs that have been developed for the SLC will be available for use by physicists and operators working with the FFTB.

\section{IX.A Data Accuisition}

The FFTB will be controlled by the VAX 8800 that operates the SLC. The VAX manframe is linked to a network of distributed microprocessors through a frequencymodulated broadband communications cable. Each node of the network consists of a multibus crate that contains a single Intel 8036 microprocessor with local memory. CAMAC is driven by a Multibus (AMAC Driver (MBCD) that provides direct-memory access through a high-speed serial link. Up to fiftcen ('AMAC crates can be driven from one $\mathrm{HBCD}$. The CAMAC system conforms to IEEE 583-1982 specifications, and all of the nodules used in the FFI $B$ are either commercially available, or have previously been developed and used at SLAC'. Two microprocessors will be used for the FFTB. The CAMAC structure for the FF'TB is shown in Figures IX.1 and 2, and detailed rack and crate profiles are available. 


\section{IX.B Software}

The software facilities and applications programs that have been written for the SLC will all be available for use with the FF"TB. The VAX programning language is FORTRAN. We do not anticipate that any new soltware will be required for the microprocessors during the initial commissioning of the beasn line, but new code may be developed during later use of the facility. In particular, new fast feedback algorithms may be clesired to control the beam or lattice parameters.

Data structures for the FFTB will be incorporated into those already created for the SLC. There are three interrelated structures of importance. The dalabase contains static information necessary for the operation of the beam linc components; e.g., device characteristics, CAMAC addresses, lunctions, ct.c. Configuration files are used to store particular states of machine parameters. These fjles save directly controllable parameters, such as power supply settings, mover positions, etc. The T-malrix contains all timing information for the machine. Additions to these structures to accommodate the FFTB will be made by members of the SLC Software group.

\section{IX.C Machine Protection}

A control system exists to protect machine components from damage due to crrant steering of the beam or hardware failures. Tempcrature sensors and water flow switches are used to insure that magnets and collimators are sufficicntly cooled, and current monitors are used to insure that power is supplied to each magnet. Ionization chambers, capable of detecting average radiation levels as woll as pulse-to-pulse beam losses, will be installed along the beam line. Signals from thesc monitoms are processed by electronic discriminators to generate logic signals when adjation levels or beam losses become too high. When the average rate of beam loss is found to be sufficiont to threaten machine components the beam repetition rate is automatically reduced. Single-pulse losses that are extreme will result in a trip of the clectron gun that must be reset by an operator. The Personnel Protection System. that is described in Soction $X$ ol this roport, uses special monitors to guard against excessive maliation in areas laat are accessible to personnel. 


\section{IX.D Special Purpose Control Hardware}

Mude of the control hardware for the Flet33. and essentially all of the electronics,

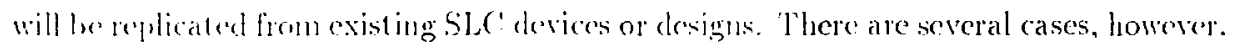
in which now designts are bejng created.

Hugnet Hoif Control

Adescription of the latedwate to be used 1.o provicle novement of the FPTB magnets is given in Section VI.E of this report. The systcon has been developed from a sinvilar onc nsed in the Slec to control the aligmment of 1 lac final triplet of magnets on each side ol the nuteraction point. The design uses thee stepping motors per magnet to generate transations in the transverse plane, and roll about the longitudinal axis of each magnet. The transwerse morement covers a full range of $\pm 2 \mathrm{~mm}$ in step sizes of $0.4 \mathrm{~mm}$. There is an option to run the system in "hall-step" mode to provide $0.25 \mu \mathrm{m}$ per step. A thousand of these motors are in use in the SLC ARCs where they have performed well.

Read-out of the position of the magnets is done in two ways, by cermet rotary por cut ioneters monnted dicctly on the stepping-motor drive shafts to verify the number wisteps taken, and precision L'DT's to read directly the position of the magnet with respect to its fixed mount. The read-out electronics, which is dispersed among three support buildings to kcep the LVDT caljles under 250 it in length, is identical to that used to control movements of collimators and magnets in the Linac, ARCs, and Final livens of the SICC:

Hligmment Contiol

Control of the alignment system has been clescribed in Section VI.D of this rejort. 


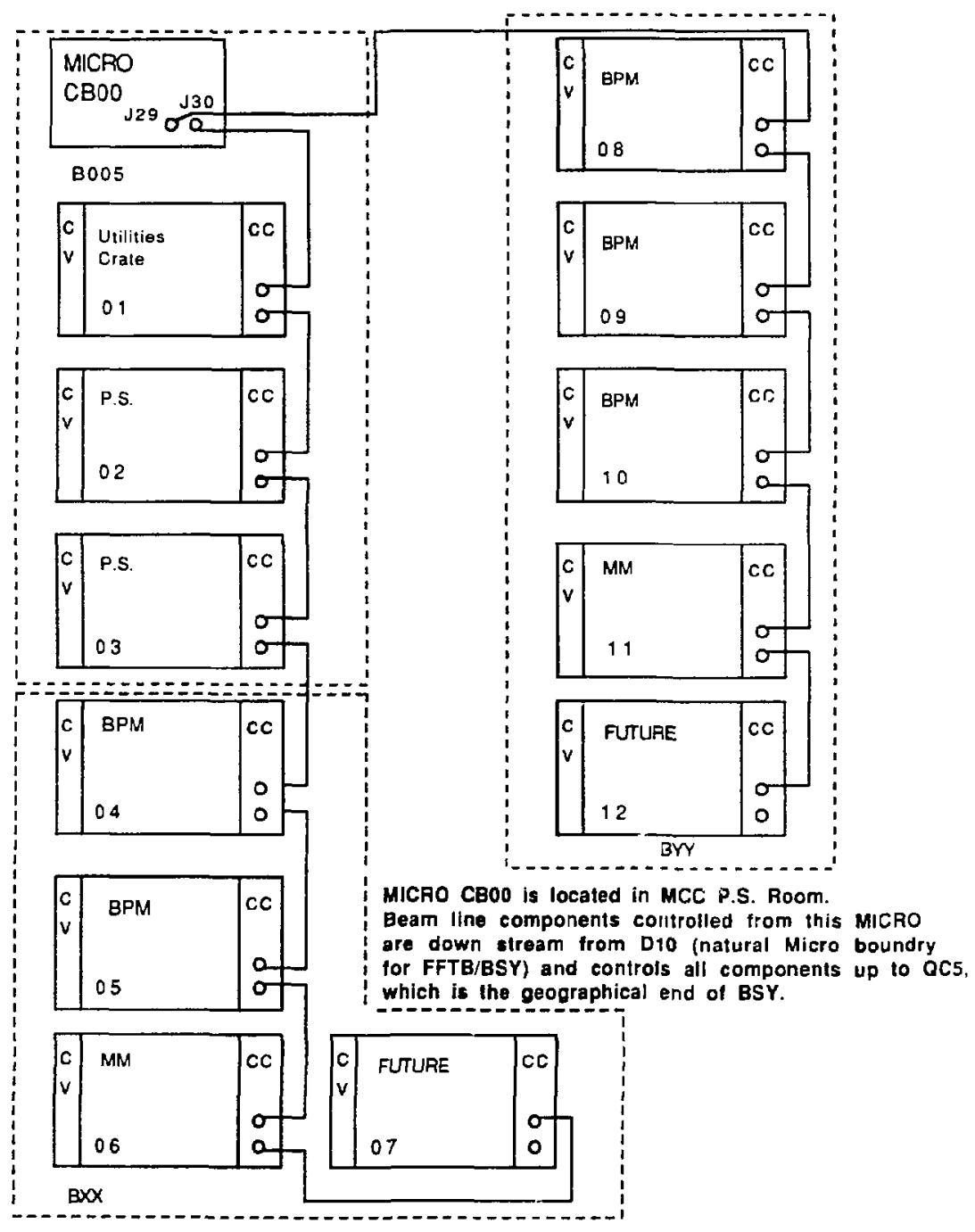

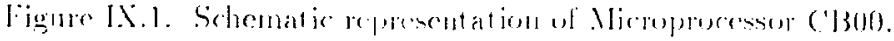




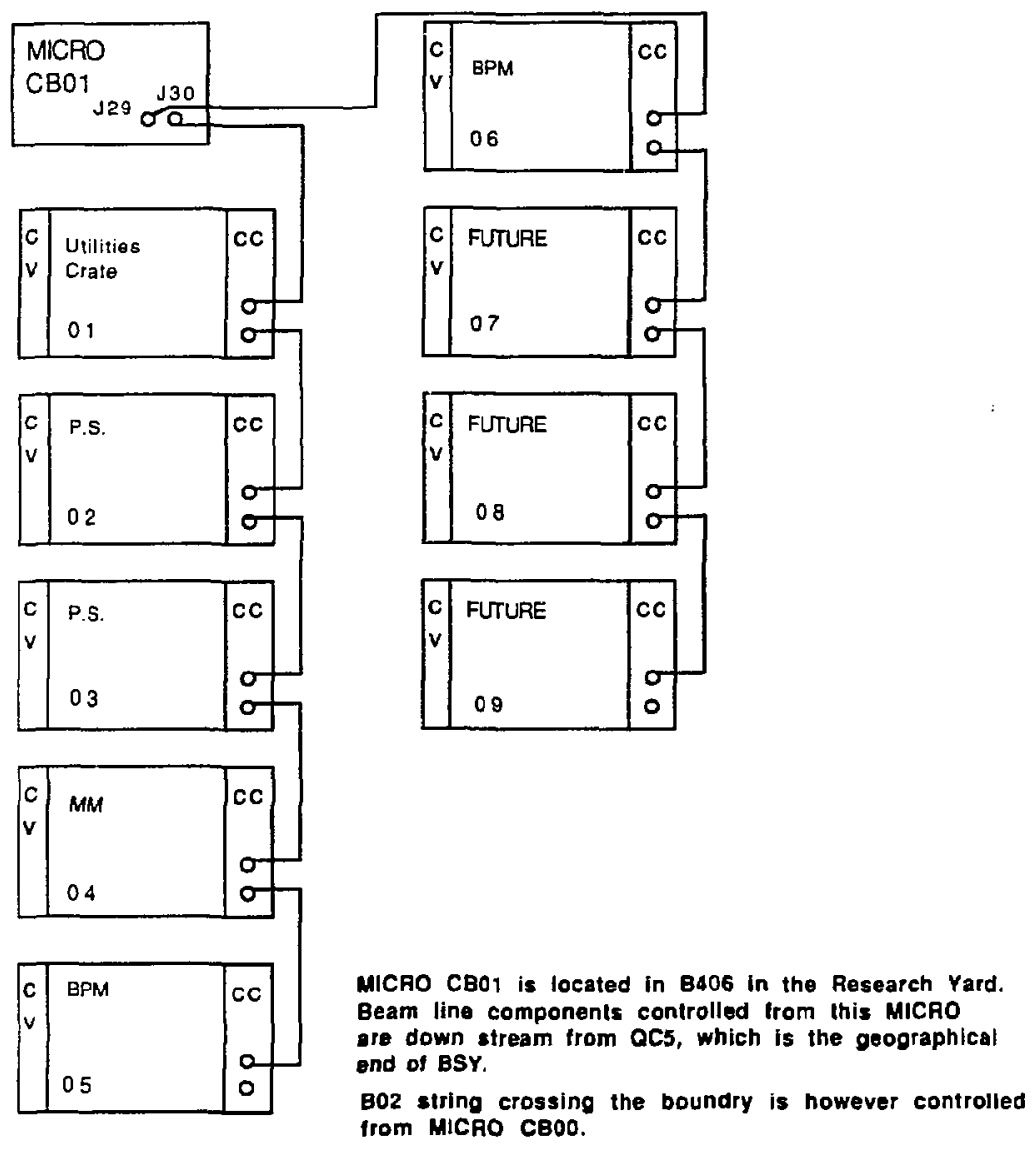

ligme IX.2. Schematic expessentation of Microprocessor CHOL.

IX 1 
X. Radiation Shielding and

\section{Personnel Protection}




\section{Radiation Shielding and Personnel Protection}

The FFTB will be housed in a shielded enclosure located inside the SLAC radiation security fence where it will be accessible only to authorized personnel. A plan view of the FFTB beam line is given in Figure X.1. Approximately 150 meters of the FFTB enclosure will be located within the confines of the Beam Switch Yard (BSY). Concrete shielding blocks will cover the beam line in this area, as shown in Figure X.2, and a thick iron shield will be installed in the C'-Line channel at the front of the FFTB (between Stations 105 and 106 in Figure X.1). This will be sulficiently thick to isolate the beam line from the remainder of the accelerator environment. Another 100 meters of the FFTB will be housed in a structure of blocks that will be erected on the concrete surface of the Research Fard.

The FFTB will use a low power beam that we anticipate operating 2 to 3 months a year. The SLC and PEP injection lines that slare the BSY will run during most of the rest of the year. The shielding for the FFTB is designed to allow access to the beam line while other beams are passing through the BSY. The Personnel Protection System (PPS-Section X.B below) will enforce three levels of access to the FFTB beam line:
No Access
Beam can be brought into the FFTB beam line.
Restricted Access
Beam can be in the BSY, but not the FFTB.
Unrestricted Access
No beams can reach the BSY.

The Eid Station A beam may run for short periods in the future. It may not be possible to maint ain access to the FFTB during these times

Sources of radiation have been identified by personnel familiar with the operation of the SLAC: linac and facilities that receive beams that pass through the BSY. Calculations of the expected radiation levels in the FFTB bean line, and design and modeling of the shielding for the FFTB, have been done by members of the SLAC Radiation Physics group. ${ }^{1}$ These designs have been reviewed by the Safoly Committee at SLAC, and the Health Physics group will monitor the levels of radiation that appear in the beam line area during periods of actual operation. 


\section{Restricted Access}

The most energetic beams at SLAC are produced tor the SLC. The FFTB shielding is designed to allow limited access to the beam line during periods of extended SLC operation. The following assumptions and parameters have been used in the design process:

1. Maximum SLC power $=150 \mathrm{kll}$ (two beans).

2. Beam loss of $30 \mathrm{~kW}(20 \%)$ at eiglt collimators located between Sectors 28 and 30 in the linac.

3. Beam loss of $7.5 \mathrm{~kW}$ on each of the BSY slits 51SL1/SL2 and 52SL1/SL2 (i.e., $10 \%$ total).

4. Occasional loss of $10 \mathrm{~kW}$ on the $\mathrm{D} 2$ dump in the C--Line.

5. Full beam loss on the BSY dumps (51/52 D1) for an indeterminate period of tine.

6. Use factor of 0.7 during periods of scheduled SLC operation.

7. Occupancy of workers limited to lower level of C-line from station $105+7 s$ to the Research Yard.

8. Maximum occupancy time of $2000 \mathrm{hr} /$ year for workers during scheduled SLC: running.

With these assumptions, the shielding is designed to limit the radiation dose in the FFTB beam line as follows:

1. The dose equivalent at the surlace of the shicld. integrated over the working year (2000 hr), must be $<1$ rem.

2. In a worst case (accident). Ihe dose rquivalent rate must be less than $25 \mathrm{rem} / \mathrm{year}$.

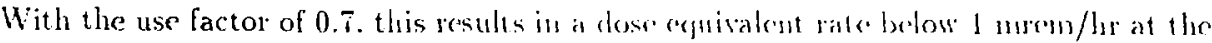
surface of the shielding in the I3SY. 


\section{No Acess}

The Beam Containment System (BCS-Section X.C below) will limit the FFTB beam to a maximum of $1 \mathrm{~kW}$ average power. This corresponds to $1 \times 10^{10} 50 \mathrm{GeV}$ electrons per pulse at 10 pulses per second. No access to the beam line can be made while this beam can reach the FFTB. An active bend magnet and three independent beam stoppers must be inserted into the beam line up beam of the FFTB area before this restriction can be removed.

The following parameters and beam losses have been assumed for the operation of the FFTB,

1. Maximum FFTB Power $=1 \mathrm{~kW}$.

2. Ten Watts (1\%) continuous loss at B01 $\mathrm{A}$ bending magnet.

3. One Watt $(0.1 \%)$ continuous loss along the beam line.

4. Operation of the FFTB for 600 hours/year.

5. One-hundred percent occupancy of the Research Yard during FFTB operation. The shielding in the Researcl Yard is designed to reduce the radiation levels to less than $1 \mathrm{mrem} / \mathrm{hr}$ at the outside surface of the shielding bloclis.

\section{X.A Radiation Slielding Design}

Distributions of muons, gamma rays, and neutrons that are produced in electromagnetic showers generated by $50 \mathrm{GeV}$ electrons (or positrons) have been calculated using standard tecliniques. ${ }^{2}$ Combinations of ray-tracing and ducting models have then been used to estimate the radiation that reaches areas that are accessible to personnel. The shielding is then specified to reduce the dose ratos to the levels given above.

\section{BS' Shielding}

Nineteen meters of iron is recuired to shick the FFTB channel from muons created in collinators at the end of the linac. Sixteen meters will be installed as a single wall covering the entire lower level of the channel (i.e. $12 \mathrm{ft}$ wide and $9.5 \mathrm{ft}$ high) up bean of Station $105+7 S$. The accelerator waveguide structure and iron of the magnets up beam of the FFTB account for the remainder of the muon shiclding. 
Concrete blocks will be used to shield against gamma rays and neutrons. These blocks will cover the beam line components from the beginning of the BSY to the ESC wall at a height of $8 \mathrm{ft}$ to $9.5 \mathrm{ft}$. The thickness of the roof blocks in the BSY varies between 1 to $2 \mathrm{ft}$.

1. Source-shield roof blocks (up beam of $\mu$-shield):

One-foot thick concrete slabs will be placed along the BSY channel ledge. starting as close to Station $102+20$ as possible and running the entire length to the start of the muon shield. This is to attenuate radiation fron SL1/SL2, D10, and D2, as well as any additional sources that have yet to be identified.

2. FFTB channel roof blocks (down beam of $\mu^{- \text {shicld): }}$

Two-foot thick concrete slabs should be placed along the BSY channel ledge, starting near Station $105+78$ and running the entire length to where the BSY narrows (Station $107+75$ ). One-foot thick concrete roof blocks will cover the remaining FFTB channel from Station $107+75$ to the BSY egress. No gaps are allowed in the FFTB channel roof shield, which also acts as a personne] barrier for the BSY.

3. BSY (upper) egress shield:

A nine-ft thick wall will be stacked at the end of the BSY and above the exit of the FFTB channel to prevent radiation from escaping into the Research Yard.

\section{Rescarch Yard Shielding}

The shielding in the Research Yard will be constructed from shielding blocks that will be removed from the SLC Collider IIall once the SLD replaces the Mark II. The sicles of the FFTB shielding will be 4 [t-1 ind thick and the roof will be $3 \mathrm{ft}-3$ inches thick. This structure ends jin a benm plug composed of iron and concrete that will sutround the FFTB beam clump. 


\section{Enrestricted Access}

Linrestricted access to the FFTB will be: allowed during periods of time when no beams can be transported to the BSY arca. A special case of unresuricted access to the beam line will be established during the construction of the FFTB by initially plugging the holes in the front steel shield that would normaliy be open for the beam pipe and laser aligument pipe. This will allow the construction of nearly all of the FFTB during periods when the SLC and PEP are running.

\section{X.B Persomnel Protection System (PPS)}

The PPS system will be installed in two phases. It will first be necessary to install sufficient controls to assure that the FFTB arca is safe during the construction of the new beam line. The second phase consists of the construction and installation of security gates and monitors to allow the beam line 10 be used by experimenters.

During the first phase extrancous equipment. will be removed from the old C-Beam and End Station C PPS gate. These will be stored for later use. New components will be installed to insure the radiation safely of the FF'CB alea. The D2 dump, two stoppers ST60 and ST61, and a disaster monitor will be installed up beam of the muon shield, and two BSOICs will be installed down beam of the muon shield in the FFTB area itself. This will be sufficient to prevent beam from striking the muon shield, and will shutoff the beam if the radiation levels in the FFTB channcl should exceed the limits prescribed above.

For the second phase, a PPS system compatilyle witl existing equipment and safety standards will be installed, tested and cerified. The $s$. " $\mathrm{cm}$ vill protect the entire length of the FFTB. One entry module will be installed midway along the extension of the beam line onto the Research l'ard. Emergency-Off but ons. Scarch-Reset buttons, audible and visible warning systems, electrical hazard protection. and interlocks on movable shielding blocks will be installed. A disaster monitor will be placed hehind the FFTB dump, and two additional BSOIC's will be mounted in strategir locations to monitor radiation levels outside of the FFTB shiclding. The local alectronics for the PPS system will reside in the 406 support building away from high radiation and adverse temperature conditions. 
This complete system will be integrated into the SLAC-wide PPS system, and will be controlled from the SLAC Main Control Cenler.

\section{X.C Beam Containment System (BCS)}

The BCS system for the FFTB must protect the dump D2 when beams are stopped up beam of the muon shield, and must assure that beams in the FFTB are properly transported to, and stopped by, the FFTB dump or ST 62 stopper.

The bean power on the D2 dump will be limited to $15 \mathrm{kll}$ by two toriods. 13 and 14, and ion chambers IC104A and IC10 13. The toroid signals will be processed by electronics capable of detecting both eiectrou and positron bunches cren if they are produced on the same machine pulse. The beam stoppers ST60 and ST61 that are up beam of the muon steel will be protected by jon chambers IC' 65 and IC' 66.

Additional toroids in the FFTB bean line will hinit the power delivered to the FFTB dump to $1 \mathrm{~kW}$. Ion clambers will also be used to protect the dump and the intermediate stopper ST62 located down beam of the muon stcel in the FFTB. 


\section{References}

1. We thank E. Benson and R. Nolson for their calculation of these radiations and help with the design of the sliclding for the PF'TB.

2. National Council on Radiation Protection and Measurements, NCRP-Report No. 51. March 1, 1977;

T. M. Jenli, is, Nucl. Instr. Meth. 159 (1979) 265:

W. R. Nelson and K. R. Kase, Nucl. Instr. Meth. 120 (1974) 401;

H. DeStaebler. T. M. Jenkins and W. R. Nelson, "Shielding and Dosimetry," in The Stanford Two Mile Acclerator," R. B. Neal. editor (Benjamin, 1968). 


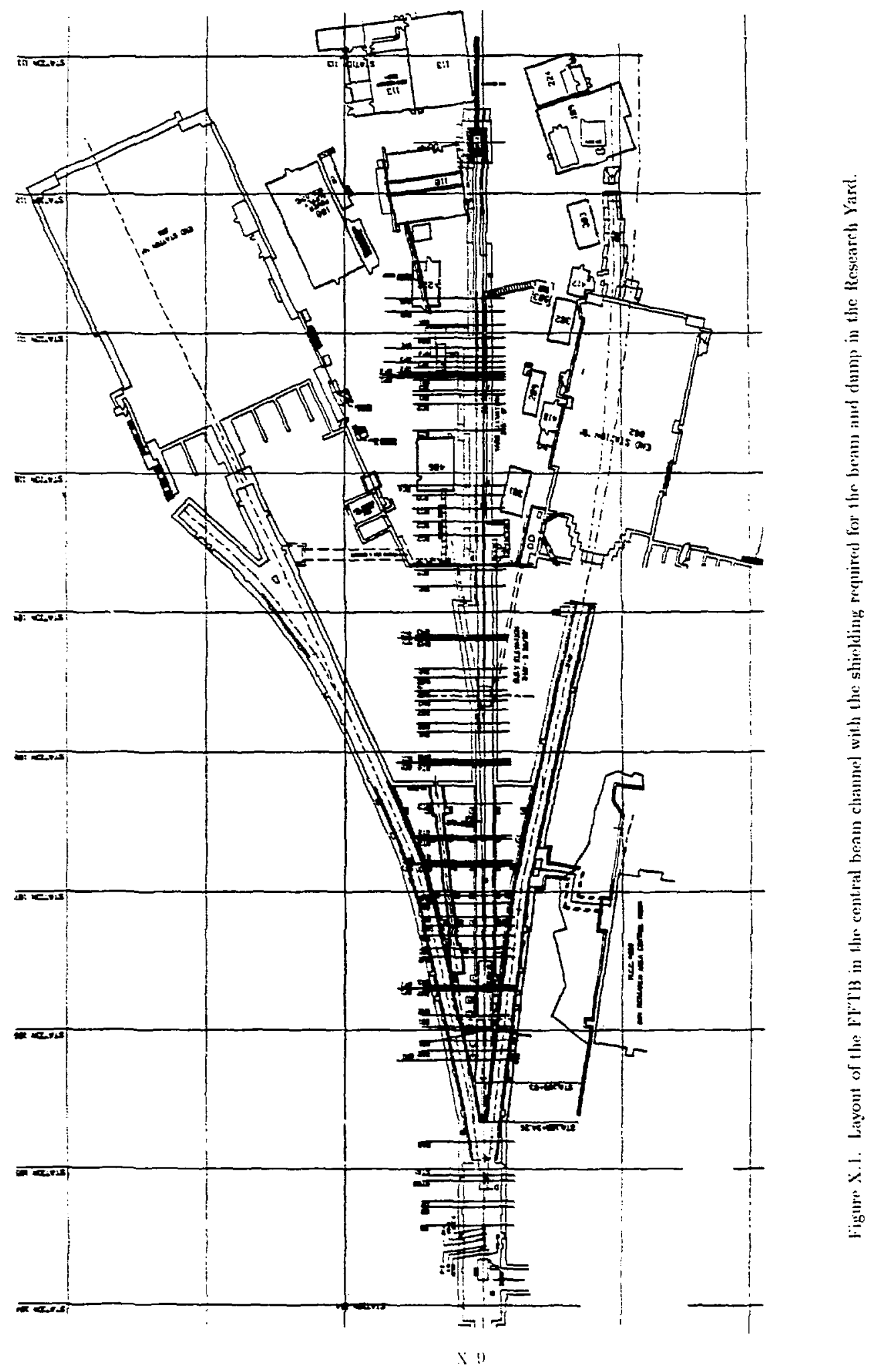




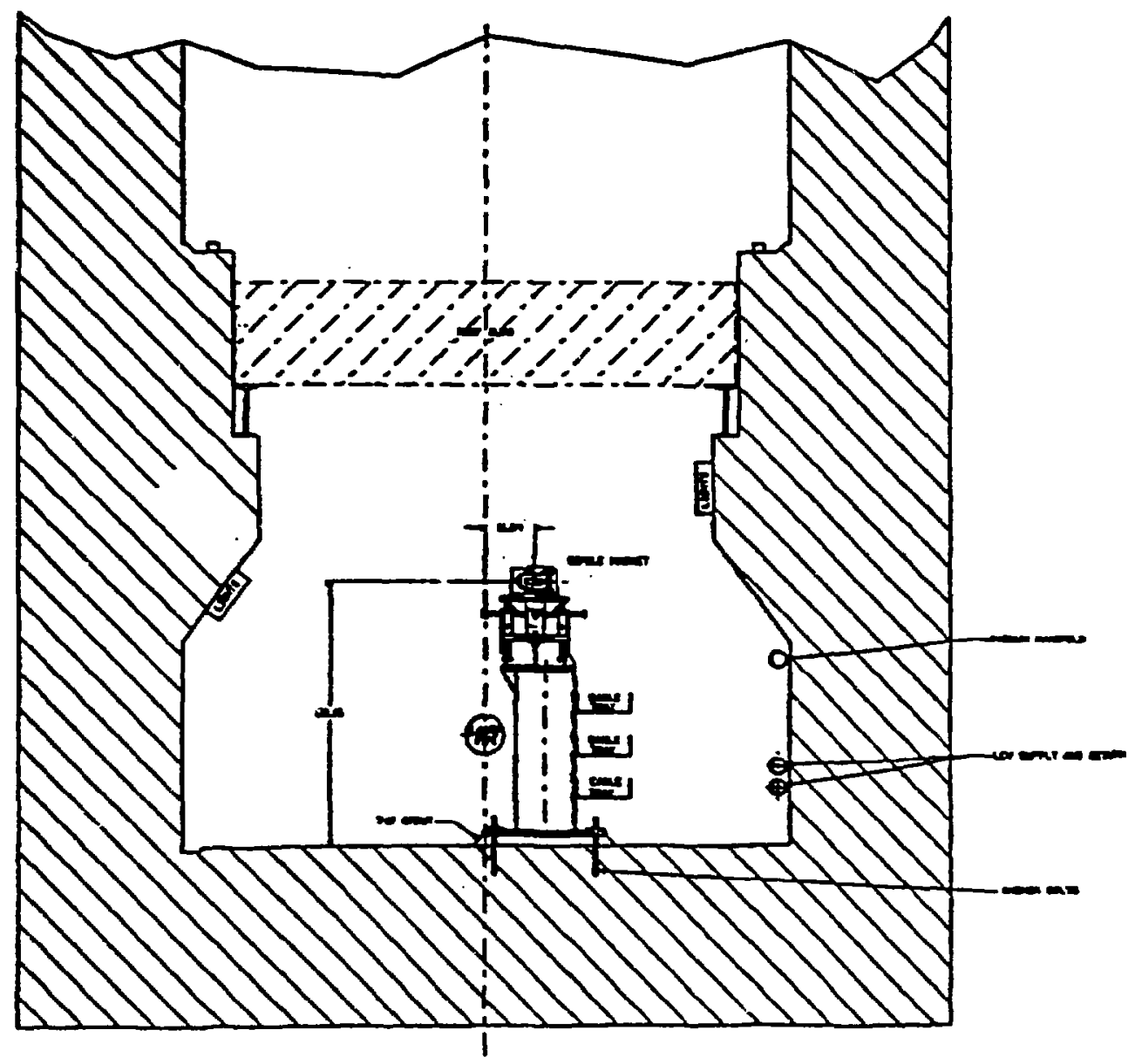

Figure X.2. Shielding of the beam line in the Bram Switch Yard area. 
XI. Infrastructure 


\section{Infrastructure}

The infrastructure associated with the FFTl3 is described in this section. The support buildings, utilities, cable plant, and installation fixtures needed for the FFTB are similar to those for other lines that have been constructed in the Research Yard in the past. In some cases, structures must be removed to make way for the FFTB, and in other cases, structures will be reused as part of the new beam line.

What is left of the old C-Beam that has existed in the channel to be occupied by the FFTB must removed, as must parts of the SPEAR injection line (which has been replaced by the new SSRL dedicated injector). Buildings 109 and 110 in the Research lard must be modified, and Bldg. 406 will be moved and refurbished to house some of the FFTB power supplies and electronics. A section at the back of the Main Control Center must be cleaned out to make room for the remainder of the FFTB power supplies. Two additional support shelters must be established on top oi the linac berm to house electronics racks for the FFTB instrumentation. These structures and the pattern of cable raceways to be installed for the FFTB are shown in Figures XI.1-3.

\section{XI.A Beam line Utilities and Structures}

The radiation shielding for the FFTB has been discussed in Section X. Electrical $A C$ power for outlets and lighting within the shielded area will be supplied from the electrical vaults near End Stations A and B. The FFTB magnets represent a total power load of less than $500 \mathrm{liW}$.

Care will be taken to control the infiltration of air into the beam line area to minimize diurnal temperature cycling. Ventilation will be provided during periods of personnel access to the beam line. A low lesel of air flow will be provided to meet health recuirements for workers in the area, and a systcm capable of purging the area of smoke in an emergency will also be installed. Smoke detcctors and a wet-pipe sprinkler system will provide additional protection againsi damage or injury due to fire.

Several of the blocks that make up the rool of the shielding in the Research Yard will be removable to facilitate installation of the bean line components. A light crane (1 ton) will be luung from railings attached to beans that straddle the ledge of the 
beam line channel. This will be sufficient to lilt all of the magnets, vacuum pipes, and instruments that will be installed along the beam.

The support structures for the magnets will be mounted on iron plates grouted to the concrete floor. The supports for the quadrupole and sextupole magnets will be made of a quartz-epoxy aggregate (trade name Anocast from the Anorad Corporntion), and the dipole magnets will be mounted on iron stands that were removed from the C-Beam. These are shown in Figures XI.4 and XI.5.

The low-conductivity cooling water (LCW) for the magnets will be fed from the existing system in the Research Yard. The total LCW recpuirement for the FFTB is $200 \mathrm{gpm}$, to be delivered at a pressure clifferential across the magnets of 100 psi. The LCW will be supplied with a manifold discharge pressure of $260 \mathrm{psi}$, with valves set to produce the required differential pressure. The piping in the tunnel is designed to provide a nearly constant differential by "crossing" the supply and return. The manjfolds are equipped with welded, threaded at tach points for individual isolation valves, and all piping is stainless steel. The supply and return headers will be installed the length of the the FFTB, and will be connected to the magnets through low-conductivity flexible hoses.

\section{XI.B Support Buildings}

The layout of the racks and floor-mounted power supplies for the FFTB are shown in Figures XI.2 and 3. Rack and CAMAC crate profiles for the system are available. Each of the support buildings and areas is serviced by AC power with sejarate transformers for the DC power supplies and electronics racks. Heating, ventilation. and air conditioning: is provided for the support buildings to balance the heat load from the power supplies and electronics raclis.

\section{XI.C Cable Plant}

Cables will be run in 4-inch deep ladder-type steel cable trays or ofluer raceways from point of origin to temination. Barriers in the cable trays or separate cable trays will be utilized to cary conductors for different functions: c.g. DC'. instrumentation. AC: power, etc. 
To minimize cable lengths for the Bean Position Monitor circuits and other systems, some of the electronics will be localed in the two shelters on the surface of the berns above the BSY. A 27 -inch diameter vertical penetration adjacent to each of the support buildings will carry cables to cable trays in the interior of $1 \|_{1}$ BSY and to beam line equipment. Inside the vertical penetrations. conduits will separate conductors for different functions. Kellem, or similar cable grips will support the vertical load of the cables. Provisions will be made at the surface in the vicinity of the penetrations for shielding of the penetrations since they represent a straight path to the interior oi the BSY.

Existing cable trays between the Main Control Building ( $\mathrm{MCC}$ ) and the BSY will be utilized for cables between those two locations.

\section{Laige DC: Cables}

DC' cables will be compact-sector alumimum, with XHHW insulation. Connections to the magnets will be made utilizing short jumpers from terminal cabinets immediately adjacent to the magnets to minimize the weight of cables on the magnet terminals. Terminal cabinets will be provided at the power supply end for installation of transductors on the DC circuits prior to connections to the Power Supplies. See Table XI.1 for a listing of the sizes of these cables.

\section{Trim and Steering Magnet Cables}

To aid in the problem of maintaining proper voltage levels for the trim and steering magnet cables, trunk cables of number- 6 conductors will be run frorn terminal cabinets near the power supplies to terminal cabinets in central locations near the magnets. From the teminal cabinets to the power supplies and to the magnets, smaller sized conductors will be installed. The terminal cabinets will also be helpful when isolating circuits and for trouble-shooting shorts, or other cable problems. Spare circuits will be included in the trunks.

Wiring for the trim and steering magnet trunks and jumpers will be copper with XHHW insulation. 


\section{Instrumentation and Control Cables}

A wide variety of cable types will be utilized for the instrumentation and control systems. Where practical, cables will be preassembled witl $]_{1}$ connectors, as complete units. In many cases cable connectors will be installed in the field.

Cables for the Beam Position Monitors and possibly the Wire Position Monitors will be phase-matched in the SLAC shops and installed as completed issemblies.

Multi-conductor instrumentation and control cables will be type TC (tray cable), with overall shield. 


\begin{tabular}{|c|c|}
\hline \multicolumn{2}{|c|}{ Table XI.1 Magnet Power Supply Cable List } \\
\hline Magnet & Wire Size \\
\hline B01A-THROLIGI F & $2-250 \mathrm{MCM}$ \\
\hline BOIA-THROLGH F & 2-250 MCM \\
\hline SF1A \& SF1B & NUMBER $4 / 0$ \\
\hline SD1A \& SD1B & NUMBER $4 / 0$ \\
\hline QS1 & \\
\hline Q5 & NUMBER 6 \\
\hline Q6 & NUMBER 2 \\
\hline QA0 & NUMBER 2 \\
\hline QA1 & $300 \mathrm{MCM}$ \\
\hline QA2 & NUMIBER $2 / 0$ \\
\hline $\mathrm{QN2} 2 \mathrm{QN2}, \mathrm{QN} 1, \mathrm{QN2}$ & $300 \mathrm{MCM}$ \\
\hline $\mathrm{QN} 3,1 \mathrm{~N} 3, \mathrm{QN} 3$ & NUMBER $1 / 0$ \\
\hline $\mathrm{Q}^{\prime} \mathrm{\Gamma} 1$ & NUMBER $4 / 0$ \\
\hline QT2 & NUMBER $3 / 0$ \\
\hline QT2 & NUMBER $3 / 0$ \\
\hline$\overline{Q^{\prime} \mathrm{T} 3}$ & $300 \mathrm{MCM}$ \\
\hline QM3 & NUMBER $1 / 0$ \\
\hline QN1,QN2,QN1,QM3,QM3,QN1 & $300 \mathrm{MCM}$ \\
\hline QC5 & $250 \mathrm{MCM}$ \\
\hline QC4 & NUMBER 6 \\
\hline QC3,QC2,QX1,QN3 & $500 \mathrm{MCM}$ \\
\hline
\end{tabular}




\begin{tabular}{|c|c|}
\hline \multicolumn{2}{|c|}{ Table XI.1 Magnet Power Supply Cable List Continued } \\
\hline Magnet & Wire Size \\
\hline QP1A,QP1B & $300 \mathrm{MCM}$ \\
\hline QP2A,QP2B & $300 \mathrm{MCM}$ \\
\hline QP3A,QP3B & $2-250 \mathrm{MCM}$ \\
\hline QP4 & NUMBER 4/0 \\
\hline QP5 & $2-250 \mathrm{MCM}$ \\
\hline BO3A,BO3B & $300 \mathrm{MCN}$ \\
\hline BO4A,BO4B & $300 \mathrm{MCM}$ \\
\hline BO5A,BO5B & $3-350 \mathrm{MCM}$ \\
\hline QS2 \\
\hline
\end{tabular}




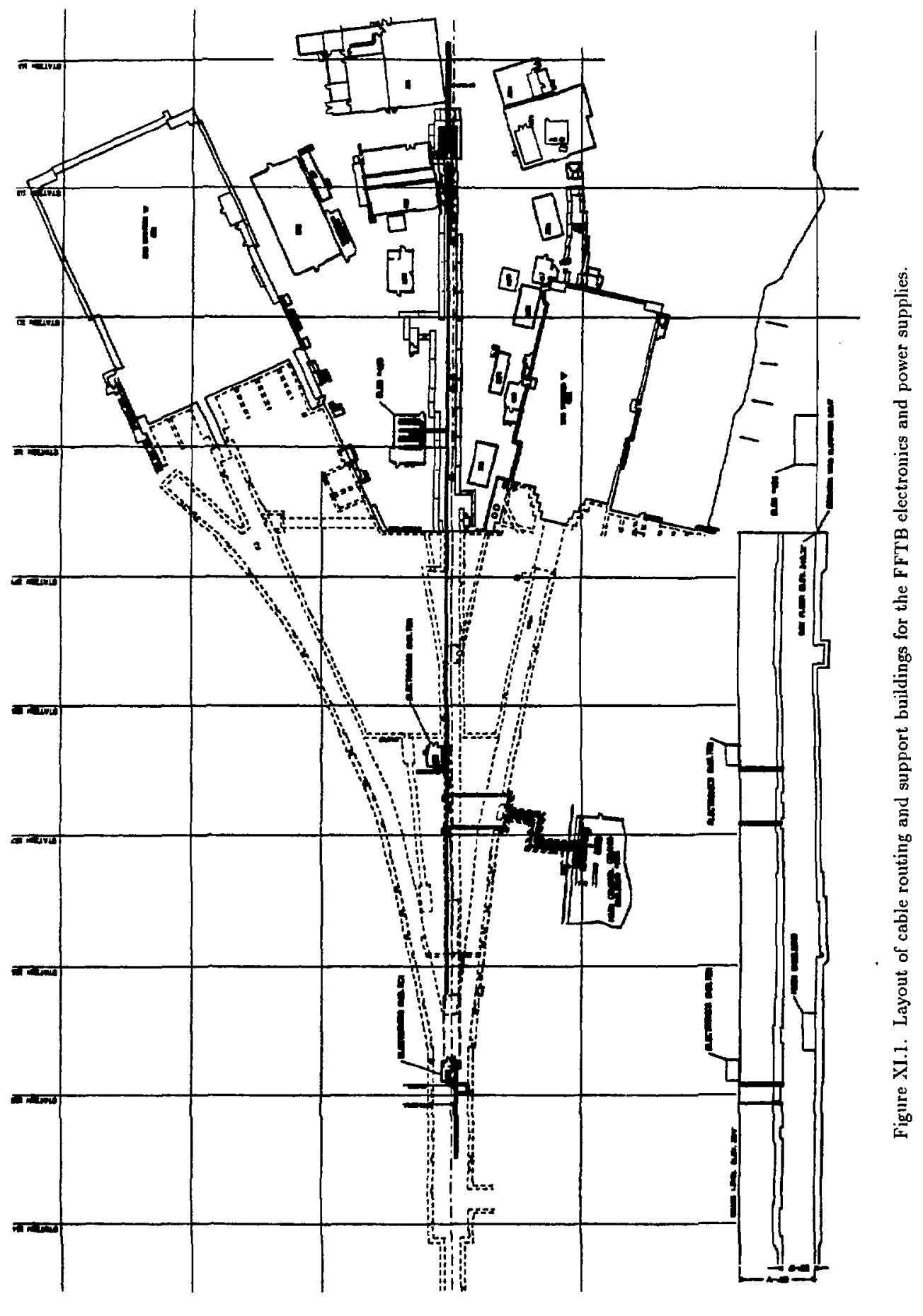



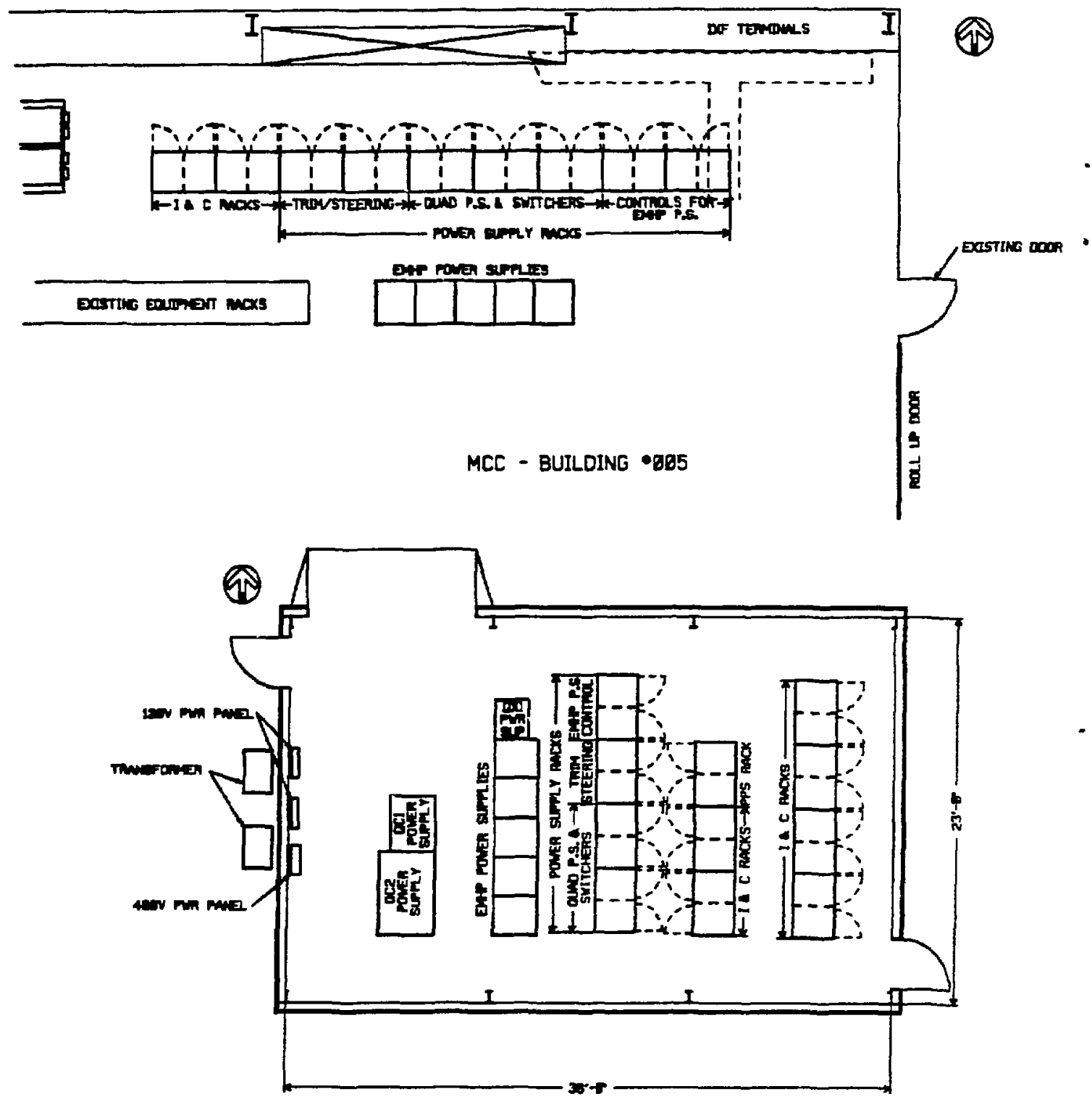

BUILDING 496

Iigure XI.2. Layout of power supplies and racks in the FFTB area of $\mathrm{MCC}$ and Building 406. 


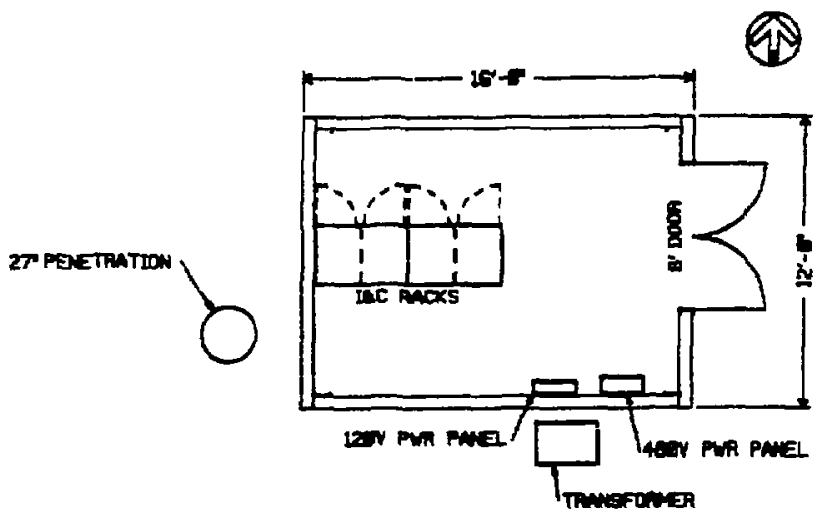

BUILDING AT STA. 105+88

(TOP OF BEAM SWITCHYARD)

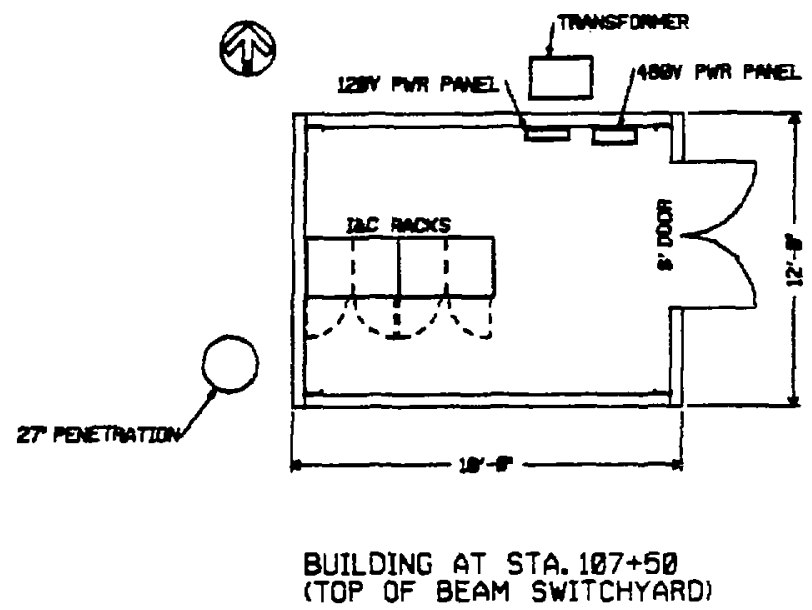

Figure XI.3. Layout of electronics racks in the support buildings on top of the Switcliyard berm. 


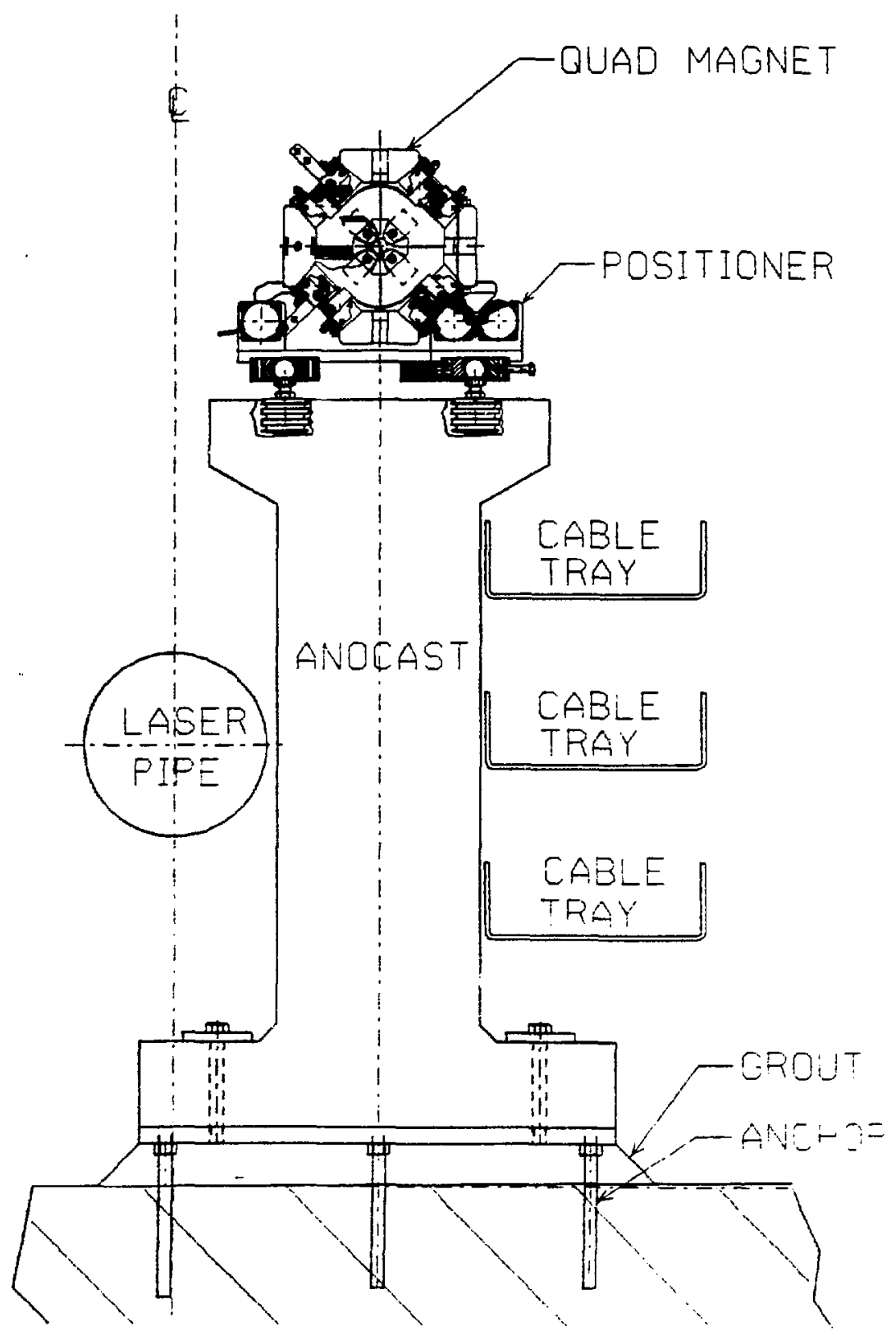

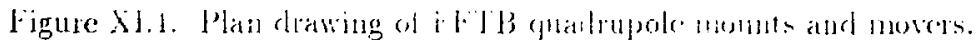




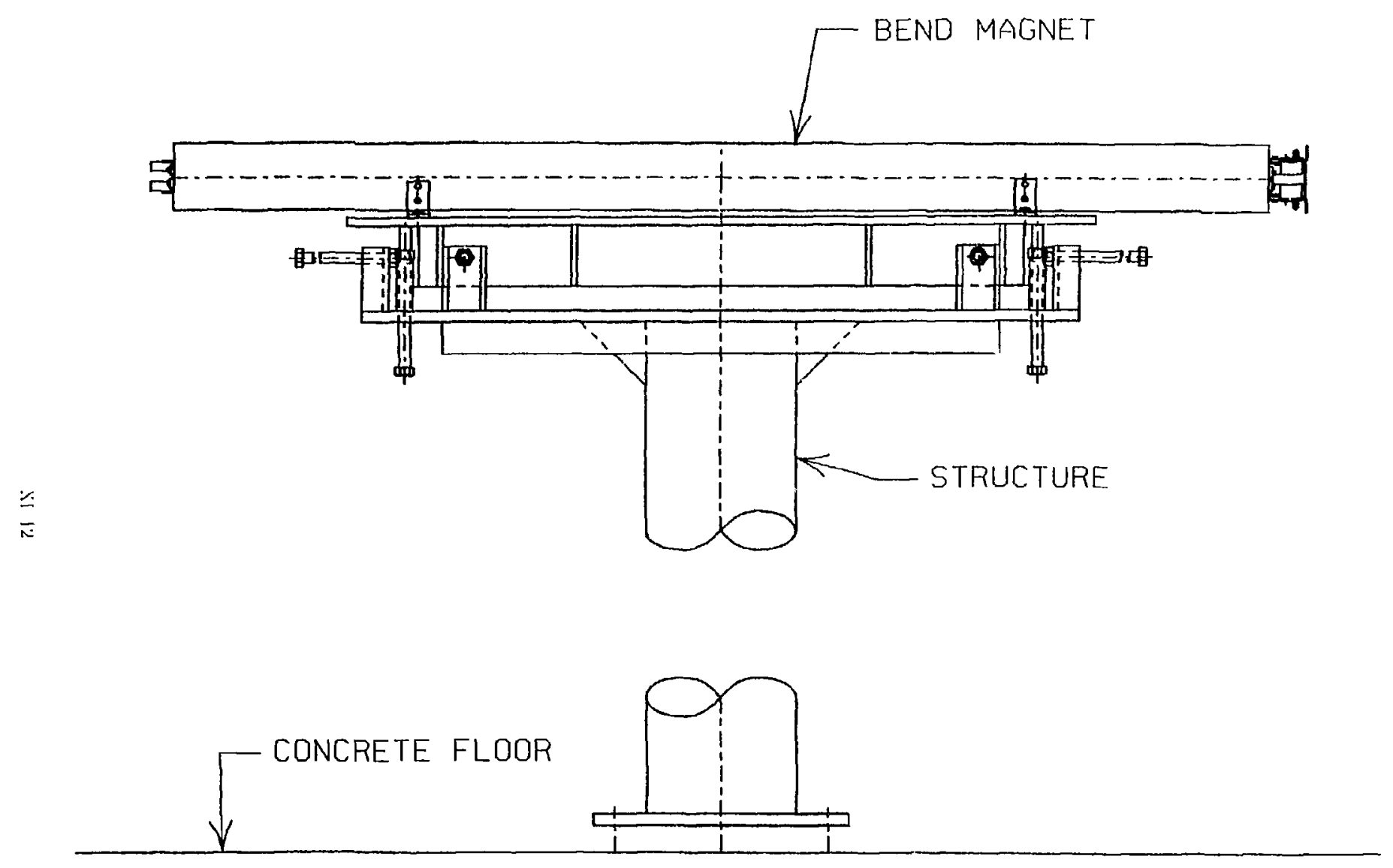

Figure XI.5. Plan drawing of FFTB dipole mounts 


\section{Administration}




\section{Administration}

The FFTB is being constructed by an intemational collaboration of engineers and physicists. The members of this collaboration are given in Table XII.1. The spokespersons for the Collaboration are D. Burke and E. Paterson of SLAC. Memoranda of Cuclerstanding have been signed that describe each institution's participation in the FFTB Collaboration.

The FFTB Collaboration is responsilsle for the construction and installation of the FFTB. The Project Manager and Project Engincer for the FFTB are D. Burke and B. Denton. The FFTB Collaboration will commission the FFTB and retain initial use of the beam line for a time after its construction to concluct various experimental investigations. It is expected that the FFTB will then becone a facility for use by the scientitic community:

\section{XII.A Budget}

Each institution participating in the FFTB Collaboration will fund all normal opcrating expenses (such as salaries, administrative support. and travel) of its personnel.

For reasons of standardization and system incgration, some material or instrumentation may be supplied by SLAC, otherwise each institution will furnish all material neccssary for the fabrication and testing of components as agreed in the Memoranda of Cuclerstanding and clescribed in this report.

The funcls required from the U.S. DOE to supprort the responsibilities of SLAC in the FF.FB Collabonation are summatized in Table XIl.2. Costs being borne by other participating institutions represent approsimately St.gN (USF' 1990 dollars with no (ontingency).

\section{XII.B Schedule}

It is the intention of the FFTB Coltaboration to complete the fabrication, installation. and inspection of 1 , EFTB components by the Fall of calender year 1992. The "Level 3 " schedule for completion of the project is shown in Table XII.3. 


\section{Table XII.1 The FFTB Collaboration}

M.Berndt, V.Bressler, K.Brown, F.Bulos, Ki.Bunnel,

D.Burke, J.Cobb, B.Denton. G.Fischer, F.Halbo, R.Helm,

P.Ilolik, J.Irwin, K.Jolnıson, A.Odian, E.Paterson,

D.Plouffe, S.Rokni, R.Ruland, R.Ruth, F.Villa.

S.Wagner, D.Wal\%, II. Walz, S.Williams

Stanford Linear Accelerator Center

Stanford, California, LSA

H.Hayano, N.Ishihara, T.Matsui, H.Nakayama.

K.Oide, N.Yamanoto

National Laborntory for High Encrgy Physics

Tsukuba, Ibaraki-ken, Japan

J.Buon, R.Couchot, J.Coulon, J.Jeanjean, F.Lediberder. V.Lepeltier, H.Ngoc, J.Perez-y'-Jorba, P.Puzo. G.Roy

Laboratoire de l'Accelcrateur Lincaire

Orsay, France

F.Loefler, F.Peters. W.Sclwarz, G.Voss

Deutsches Elelitronen-Signcheroton DESY

Hambing. Gicrmany

R.Richter, R.Setles. U'.Stjerlin, J.Thlomas

Max-Plancli-Instilute fïr Physili

Munich, Cirmmany

V.Aleksandrov, V.Balakin, Yu.lioisin. I: Mrdjidzade,

A.Mikhailichenko. Yóvaliane

Institule for Suctrar Physirs. Sibrran Division

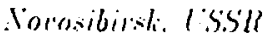


Table XII.2 U.S. DOE S'pport for FFTB

\begin{tabular}{|c|c|c|}
\hline & Item & Funds (FY90 K\$) \\
\hline 1.1 & Conventional Facilities & 2.884 \\
\hline 1.2 & Magnets & 292 \\
\hline 1.3 & Bean Devices & 330 \\
\hline 1.4 & Vacuum & 667 \\
\hline 1.5 & Magnet and Device Supports & $i 64$ \\
\hline 1.6 & Alignment & 1760 \\
\hline 1.7 & Power Supplies & 1101 \\
\hline 1.8 & Beam Containment and PPS & 404 \\
\hline 1.9 & Cable Plant & 834 \\
\hline 1.10 & Controls & 1165 \\
\hline 1.11 & Installation & 791 \\
\hline \multirow[t]{7}{*}{1.12} & Management & 399 \\
\hline & Estinzated Total & 11,391 \\
\hline & Contingency (@25\%) & 2,848 \\
\hline & Escalation ${ }^{(a)}(@ 6 \%)$ & 974 \\
\hline & Total Cost & 15,213 \\
\hline & Offscts & 930 \\
\hline & DOE Support & 1.1 .283 \\
\hline
\end{tabular}

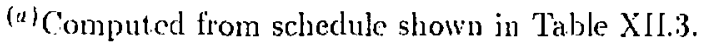


TA B LE X II.3 FFTB ACTIVITY DURATIONS

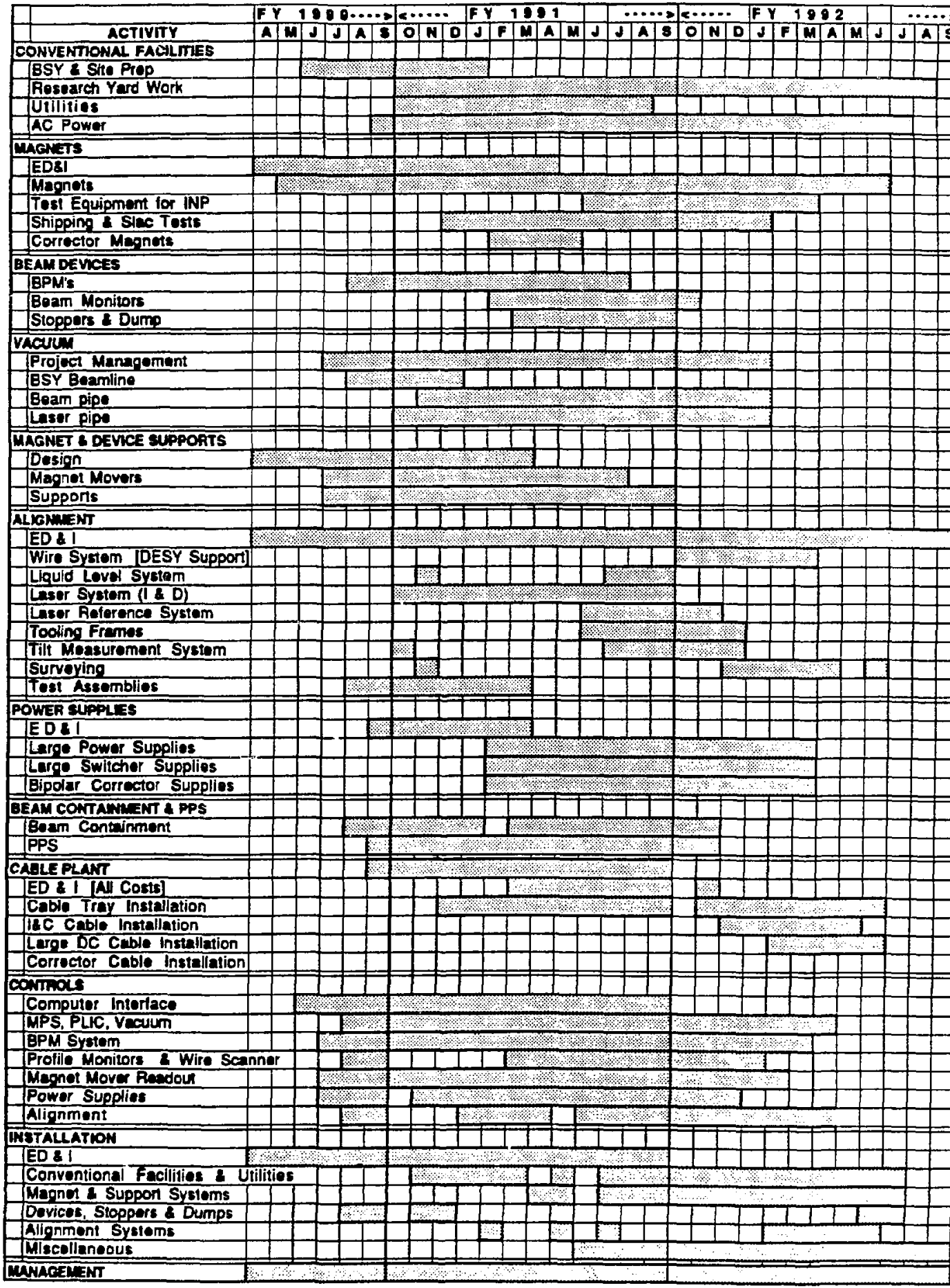

